\title{
Oxidative Amidation of Amines in Tandem with Transamidation: A Route to Amides using Visible-Light Energy
}

Jyoti Nandi, Matthew Z. Vaughan, Arturo León Sandoval, Joshua M. Paolillo, and Nicholas E. Leadbeater*

Department of Chemistry, University of Connecticut, 55 N. Eagleville Road, Storrs, Connecticut 06269, USA

\section{Supporting Information}

Energy values for transition-state and ground states

Coordinates for transition-state and ground states

${ }^{1} \mathrm{H},{ }^{13} \mathrm{C}$, and ${ }^{19} \mathrm{~F}$ NMR Spectra of Synthesized Compounds 


\section{COMPLETE CITATION FOR GAUSIAN 16}

Gaussian 16, Revision C.01, Frisch, M. J.; Trucks, G. W.; Schlegel, H. B.; Scuseria, G. E.; Robb, M. A.; Cheeseman, J. R.; Scalmani, G.; Barone, V.; Petersson, G. A.; Nakatsuji, H.; Li, X.; Caricato, M.; Marenich, A. V.; Bloino, J.; Janesko, B. G.; Gomperts, R.; Mennucci, B.; Hratchian, H. P.; Ortiz, J. V.; Izmaylov, A. F.; Sonnenberg, J. L.; Williams-Young, D.; Ding, F.; Lipparini, F.; Egidi, F.; Goings, J.; Peng, B.; Petrone, A.; Henderson, T.; Ranasinghe, D.; Zakrzewski, V. G.; Gao, J.; Rega, N.; Zheng, G.; Liang, W.; Hada, M.; Ehara, M.; Toyota, K.; Fukuda, R.; Hasegawa, J.; Ishida, M.; Nakajima, T.; Honda, Y.; Kitao, O.; Nakai, H.; Vreven, T.; Throssell, K.; Montgomery, J. A., Jr.; Peralta, J. E.; Ogliaro, F.; Bearpark, M. J.; Heyd, J. J.; Brothers, E. N.; Kudin, K. N.; Staroverov, V. N.; Keith, T. A.; Kobayashi, R.; Normand, J.; Raghavachari, K.; Rendell, A. P.; Burant, J. C.; Iyengar, S. S.; Tomasi, J.; Cossi, M.; Millam, J. M.; Klene, M.; Adamo, C.; Cammi, R.; Ochterski, J. W.; Martin, R. L.; Morokuma, K.; Farkas, O.; Foresman, J. B.; Fox, D. J. Gaussian, Inc., Wallingford CT, 2016.

\section{ENERGY VALUES FOR TRANSITION-STATE AND GROUND STATES}

\section{ENERGY VALUES FOR PYRIDINE BASED BASES}

\begin{tabular}{|r|c|c|c|c|}
\cline { 2 - 4 } \multicolumn{1}{c|}{} & $\begin{array}{c}\Sigma \text { of electronic } \\
\text { and zero-point } \\
\text { energies } \\
\text { (Hartree/Particle) }\end{array}$ & $\begin{array}{c}\Sigma \text { of electronic } \\
\text { and thermal } \\
\text { energies } \\
\text { (Hartree/Particle) }\end{array}$ & $\begin{array}{c}\Sigma \text { of electronic } \\
\text { and thermal } \\
\text { enthalpies } \\
\text { (Hartree/Particle) }\end{array}$ & $\begin{array}{c}\Sigma \text { of electronic } \\
\text { and thermal free } \\
\text { energies } \\
\text { (Hartree/Particle) }\end{array}$ \\
\hline 2,6-Lutidine & -326.808829 & -326.801017 & -326.800073 & -326.841553 \\
\hline 3,5-Lutidine & -326.802960 & -326.795024 & -326.794080 & -326.836419 \\
\hline 2,4,6-Collidine & -366.103886 & -366.094175 & -366.093231 & -366.140055 \\
\hline Pyridine & -248.214641 & -248.210354 & -248.209410 & -248.242052 \\
\hline
\end{tabular}

\section{ENERGY VALUES FOR PYRAZOLE}

\begin{tabular}{|c|c|c|c|c|}
\cline { 2 - 5 } \multicolumn{1}{c|}{} & $\begin{array}{c}\Sigma \text { of electronic and } \\
\text { zero-point } \\
\text { energies } \\
\text { (Hartree/Particle) }\end{array}$ & $\begin{array}{c}\Sigma \text { of electronic and } \\
\text { thermal energies } \\
\text { (Hartree/Particle) }\end{array}$ & $\begin{array}{c}\Sigma \text { of electronic and } \\
\text { thermal enthalpies } \\
\text { (Hartree/Particle) }\end{array}$ & $\begin{array}{c}\Sigma \text { of electronic and } \\
\text { thermal free } \\
\text { energies } \\
\text { (Hartree/Particle) }\end{array}$ \\
\hline Pyrazole & -226.147741 & -226.143995 & -226.143051 & -226.174014 \\
\hline
\end{tabular}

\section{ENERGY VALUES FOR BENZALDEHYDE WITH LITHIUM ION}

\begin{tabular}{|c|c|c|c|c|}
\cline { 2 - 5 } \multicolumn{1}{c|}{} & $\begin{array}{c}\Sigma \text { of electronic and } \\
\text { zero-point } \\
\text { energies } \\
\text { (Hartree/Particle) }\end{array}$ & $\begin{array}{c}\Sigma \text { of electronic and } \\
\text { thermal energies } \\
\text { (Hartree/Particle) }\end{array}$ & $\begin{array}{c}\Sigma \text { of electronic and } \\
\text { thermal enthalpies } \\
\text { (Hartree/Particle) }\end{array}$ & $\begin{array}{c}\Sigma \text { of electronic and } \\
\text { thermal free } \\
\text { energies } \\
\text { (Hartree/Particle) }\end{array}$ \\
\hline
\end{tabular}


ENERGY MINIMA FOR BENZALDEHYDE WITH SODIUM ION

\begin{tabular}{|c|c|c|c|c|}
\cline { 2 - 5 } \multicolumn{1}{c|}{} & $\begin{array}{c}\Sigma \text { of electronic and } \\
\text { zero-point } \\
\text { energies } \\
\text { (Hartree/Particle) }\end{array}$ & $\begin{array}{c}\Sigma \text { of electronic and } \\
\text { thermal energies } \\
\text { (Hartree/Particle) }\end{array}$ & $\begin{array}{c}\Sigma \text { of electronic and } \\
\text { thermal enthalpies } \\
\text { (Hartree/Particle) }\end{array}$ & $\begin{array}{c}\Sigma \text { of electronic and } \\
\text { thermal free } \\
\text { energies } \\
\text { (Hartree/Particle) }\end{array}$ \\
\hline
\end{tabular}

\section{TRANSITION STATES}

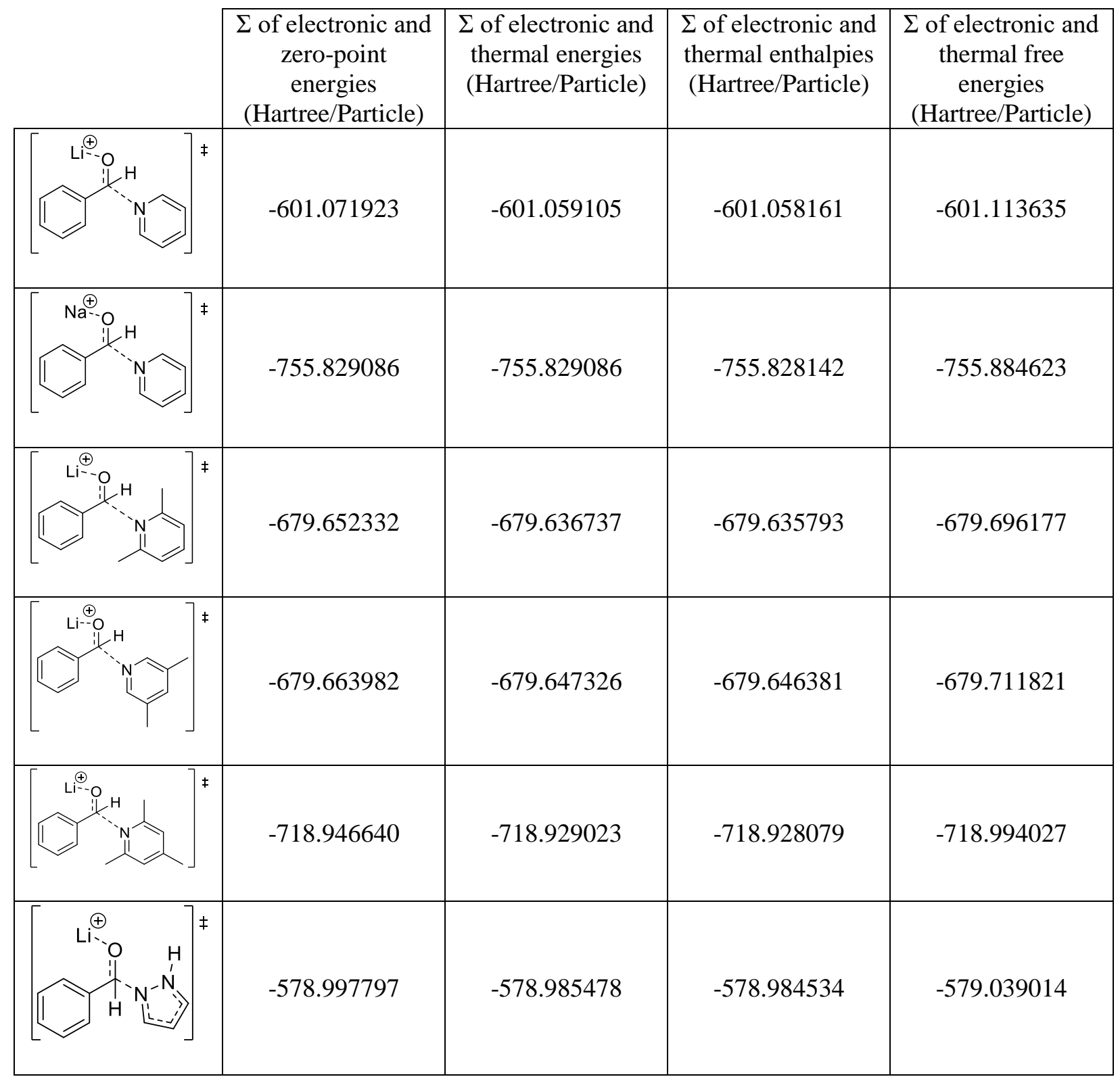


ENERGY MINIMA FOR PRODUCTS

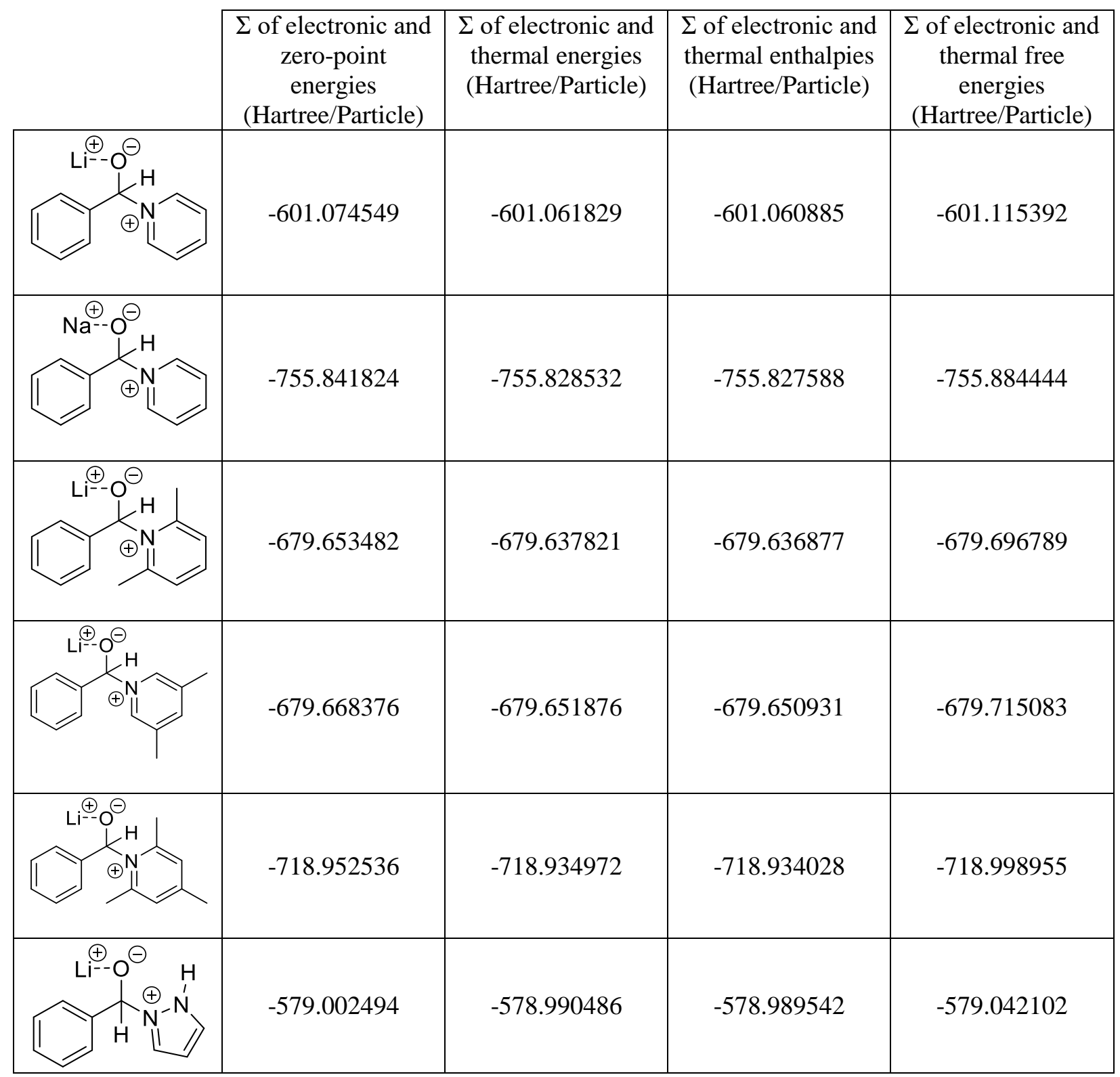




\section{COORDINATES FOR TRANSITION-STATE AND GROUND STATES}

\section{ENERGY MINIMA FOR PYRIDINE BASED BASES}

\section{2,6-Lutidine}

\begin{tabular}{lrrr} 
Coordinates (from last standard orientation): & Y \\
ATOM & \multicolumn{2}{c}{ X } & \\
O I & -1.15995500 & 0.26654600 & 0.00005400 \\
C & -1.20137300 & -1.13502900 & 0.00003500 \\
C & 0.00003200 & -1.84198300 & -0.00001700 \\
C & 1.20139600 & -1.13500600 & -0.00003200 \\
C & 1.15992200 & 0.26659200 & 0.00001400 \\
C & -0.00002300 & 0.94399300 & 0.00004500 \\
N & -2.15487000 & -1.65409600 & 0.00005800 \\
H & 2.15493500 & -1.65399700 & -0.00004600 \\
H & -2.41936200 & 1.09713200 & -0.00003800 \\
C & -2.44465100 & 1.74764400 & -0.88076800 \\
H & -3.31679100 & 0.47246600 & 0.00010900 \\
H & -2.44455000 & 1.74807100 & 0.88037400 \\
H & 2.41935000 & 1.09714800 & -0.00001200 \\
C & 2.44483700 & 1.74759300 & 0.88075600 \\
H & 3.31676200 & 0.47244800 & -0.00039800 \\
H & 2.44440800 & 1.74812100 & -0.88040200 \\
H & 0.00001500 & -2.92860600 & -0.00002400 \\
H & & &
\end{tabular}

\section{3,5-Lutidine}

\section{Coordinates (from last standard orientation):}

\section{ATOM}

\section{1}

$\mathrm{C}$
$\mathrm{C}$
$\mathrm{C}$
$\mathrm{C}$
$\mathrm{C}$
$\mathrm{N}$
$\mathrm{H}$
$\mathrm{H}$
$\mathrm{H}$
$\mathrm{C}$
$\mathrm{H}$
$\mathrm{H}$
$\mathrm{H}$
$\mathrm{C}$
$\mathrm{H}$
$\mathrm{H}$
$\mathrm{H}$
$\mathbf{X}$

$$
\begin{array}{r}
1.14436100 \\
1.21509700 \\
-0.00001000 \\
-1.21512200 \\
-1.14435000 \\
0.00002400 \\
-0.00002600 \\
2.06034300 \\
-2.06031500 \\
2.54214200 \\
3.37578900 \\
2.64781700 \\
2.64827500 \\
-2.54214700 \\
-3.37580300 \\
-2.64839600 \\
-2.64767900
\end{array}
$$

$\mathbf{Z}$

0.00005400

0.00003500

0.00001400

0.00004500

0.00005800

$-0.00004600$

0.00010900

0.88037400

$-0.00001200$

$-0.00002400$ 


\section{$\underline{\text { 2,4,6-Collidine }}$}

\begin{tabular}{|c|c|c|c|}
\hline ATOM & 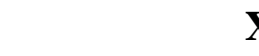 & Y & 2 \\
\hline \multicolumn{4}{|l|}{01} \\
\hline $\mathrm{C}$ & 0.70237200 & -1.15590100 & -0.00066200 \\
\hline $\mathrm{C}$ & -0.69766400 & -1.19406700 & 0.00973800 \\
\hline $\mathrm{C}$ & -1.42624100 & 0.00009400 & 0.01199500 \\
\hline $\mathrm{C}$ & -0.69751400 & 1.19415500 & 0.00961100 \\
\hline C & 0.70251800 & 1.15581900 & -0.00070200 \\
\hline $\mathrm{N}$ & 1.38635900 & -0.00008400 & -0.00627600 \\
\hline $\mathrm{H}$ & -1.21297900 & -2.15088300 & 0.01837800 \\
\hline $\mathrm{H}$ & -1.21270600 & 2.15104200 & 0.01805300 \\
\hline $\mathrm{C}$ & 1.52643700 & -2.41986600 & -0.00239200 \\
\hline $\mathrm{H}$ & 0.89764300 & -3.31445300 & -0.00288200 \\
\hline $\mathrm{H}$ & 2.17652000 & -2.44708300 & -0.88340100 \\
\hline $\mathrm{H}$ & 2.17795200 & -2.44922200 & 0.87753900 \\
\hline $\mathrm{C}$ & 1.52673400 & 2.41969100 & -0.00233600 \\
\hline $\mathrm{H}$ & 2.17733600 & 2.44661900 & -0.88295200 \\
\hline $\mathrm{H}$ & 0.89804600 & 3.31435900 & -0.00335300 \\
\hline $\mathrm{H}$ & 2.17771200 & 2.44919800 & 0.87799900 \\
\hline $\mathrm{C}$ & -2.93537400 & 0.00015900 & -0.00932000 \\
\hline $\mathrm{H}$ & -3.34216300 & 0.88693500 & 0.48602300 \\
\hline $\mathrm{H}$ & -3.30732600 & 0.00104500 & -1.04156400 \\
\hline $\mathrm{H}$ & -3.34215700 & -0.88747500 & 0.48449400 \\
\hline
\end{tabular}

\section{Pyridine}

\section{Coordinates (from last standard orientation):}

ATOM

01

$\mathrm{C}$
$\mathrm{C}$
$\mathrm{C}$
$\mathrm{C}$
$\mathrm{C}$
$\mathrm{N}$
$\mathrm{H}$
$\mathrm{H}$
$\mathrm{H}$
$\mathrm{H}$
$\mathrm{H}$
$\mathbf{X}$

-1.14457000
-1.19974400
0.00020000
1.19993800
1.14436200
-0.00020300
0.00032400
-2.06068800
-2.15857100
2.15892900
2.06030900

$$
\begin{array}{r}
-0.72277500 \\
0.67379400 \\
1.38666900 \\
0.67346500 \\
-0.72308800 \\
-1.42089600 \\
2.47299900 \\
-1.30979700 \\
1.18284500 \\
1.18220800 \\
-1.31037400
\end{array}
$$
$-0.00021800$
$-0.00012000$
0.00009000
0.00021400
0.00013000
$-0.00009800$
0.00016700
$-0.00037400$
$-0.00023100$
0.00035700
0.00018700 
ENERGY MINIMA FOR PYRAZOLE

\section{$\underline{\text { Pyrazole }}$}

\begin{tabular}{|c|c|c|c|}
\hline ATOM & & Y & \\
\hline 01 & & & \\
\hline $\mathrm{C}$ & -0.17639000 & 0.78964100 & 0.00342200 \\
\hline C & 1.20672300 & 0.78518600 & -0.00385800 \\
\hline $\mathrm{C}$ & 1.56649000 & 2.15426700 & -0.00586300 \\
\hline $\mathrm{N}$ & -0.55073600 & 2.09680600 & 0.00555900 \\
\hline $\mathrm{H}$ & -1.48623900 & 2.47297100 & 0.01028800 \\
\hline $\mathrm{H}$ & -0.90043200 & -0.01206400 & 0.00818400 \\
\hline $\mathrm{H}$ & 1.85757000 & -0.07633300 & -0.00710700 \\
\hline $\mathrm{H}$ & 2.55537300 & 2.59193000 & -0.01051200 \\
\hline $\mathrm{N}$ & 0.49606000 & 2.95007700 & -0.00027200 \\
\hline
\end{tabular}

ENERGY MINIMA FOR BENZALDEHYDE WITH LITHIUM ION

\begin{tabular}{|c|c|c|c|}
\hline \multicolumn{4}{|c|}{ Benzaldehyde with Lithium } \\
\hline \multicolumn{4}{|c|}{$\overline{\text { Coordinates (from last standard orientation): }}$} \\
\hline ATOM & $X$ & 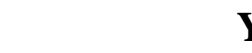 & \\
\hline \multicolumn{4}{|l|}{11} \\
\hline $\mathrm{C}$ & -2.11946300 & 3.14248400 & -0.43642900 \\
\hline $\mathrm{C}$ & -0.75598400 & 2.89047300 & -0.43807600 \\
\hline $\mathrm{C}$ & -0.28846000 & 1.56187200 & -0.31418300 \\
\hline $\mathrm{C}$ & -1.21089800 & 0.49872500 & -0.18919500 \\
\hline $\mathrm{C}$ & -2.57731500 & 0.75908800 & -0.18852400 \\
\hline $\mathrm{C}$ & -3.02818800 & 2.07784300 & -0.31198300 \\
\hline $\mathrm{H}$ & -2.48744500 & 4.15876200 & -0.53079100 \\
\hline $\mathrm{H}$ & -0.04594700 & 3.70513500 & -0.53341600 \\
\hline $\mathrm{H}$ & -0.84852900 & -0.52137100 & -0.09379000 \\
\hline $\mathrm{H}$ & -3.28846600 & -0.05436100 & -0.09300000 \\
\hline $\mathrm{H}$ & -4.09449900 & 2.28262800 & -0.31164100 \\
\hline $\mathrm{C}$ & 1.11383900 & 1.23459400 & -0.30988900 \\
\hline $\mathrm{H}$ & 1.36716100 & 0.16846400 & -0.20686200 \\
\hline 0 & 2.05851400 & 2.04561100 & -0.41142400 \\
\hline $\mathrm{Li}$ & 3.37372100 & 3.17359700 & -0.56165100 \\
\hline
\end{tabular}




\section{ENERGY MINIMA FOR BENZALDEHYDE WITH SODIUM ION}

\section{Benzaldehyde with Sodium}

\section{Coordinates (from last standard orientation):}

\section{ATOM}

11

$\mathrm{C}$
$\mathrm{C}$
$\mathrm{C}$
$\mathrm{C}$
$\mathrm{C}$
$\mathrm{C}$
$\mathrm{H}$
$\mathrm{H}$
$\mathrm{H}$
$\mathrm{H}$
$\mathrm{H}$
$\mathrm{C}$
$\mathrm{H}$
$\mathrm{Na}$
$\mathrm{O}$

\section{$\mathbf{X}$}

$$
\begin{array}{r}
-2.09483800 \\
-0.73879300 \\
-0.31060400 \\
-1.26012900 \\
-2.61873500 \\
-3.03293800 \\
-2.43270000 \\
-0.00230700 \\
-0.92701900 \\
-3.35160100 \\
-4.09231500 \\
1.09111300 \\
1.31213800 \\
3.95287700 \\
2.04189700
\end{array}
$$

3.17938800
2.88582100
1.54358700
0.50832900
0.80930500
2.14226200
4.20817200
3.67630500
-0.52390300
0.01554400
2.37870200
1.18274400
0.10468100
2.87378300
1.97680600

$\mathbf{Y}$

\section{$\mathbf{Z}$}

$-0.37026300$

$-0.40657500$

$-0.31468900$

$-0.18665200$

$-0.15065200$

$-0.24242000$

$-0.44030100$

$-0.50489500$

$-0.11611400$

$-0.05217500$

$-0.21457500$

$-0.34718200$

$-0.26809400$

$-0.60914400$

$-0.45480500$

\section{TRANSITION STATES}

Pyridine attacking Benzaldehyde with Lithium ion Coordinates (from last standard orientation):

ATOM

11

$\mathrm{C}$
$\mathrm{C}$
$\mathrm{C}$
$\mathrm{C}$
$\mathrm{C}$
$\mathrm{C}$
$\mathrm{C}$
$\mathrm{H}$
$\mathrm{H}$
$\mathrm{H}$
$\mathrm{H}$
$\mathrm{H}$
$\mathrm{H}$
$\mathrm{C}$
$\mathrm{H}$
$\mathrm{C}$
$\mathrm{C}$
$\mathrm{C}$
$\mathrm{C}$
$\mathrm{C}$
$\mathrm{C}$
$\mathrm{N}$
$\mathrm{H}$
$\mathrm{H}$
$\mathrm{H}$
$\mathrm{H}$
$\mathrm{H}$
$\mathrm{H}$
$\mathrm{O}$
$\mathrm{C}$

$\mathbf{X}$

$$
\begin{array}{r}
-2.10339800 \\
-0.74295300 \\
-0.27738800 \\
-1.18950400 \\
-2.55272400 \\
-3.00900300 \\
-2.46401500 \\
-0.03837700 \\
-0.83024900 \\
-3.25348700 \\
-4.06855800 \\
1.16061000 \\
1.40852300 \\
0.39869700 \\
0.59695500 \\
1.82265000 \\
2.80629400 \\
2.52462700 \\
1.34870700 \\
2.00817200 \\
-0.53963600 \\
-0.19425200 \\
3.77168900 \\
3.24834900 \\
2.06571100 \\
3.06285300
\end{array}
$$

Y

$-0.36495800$

$-0.20495300$

$-0.10613300$

$-0.17051800$

$-0.33953300$

$-0.43456100$

$-0.43478400$

$-0.14092300$

$-0.10628000$

$-0.40080400$

$-0.56398600$

0.02303500

0.07702900

3.00930300

4.38917700

4.88472400

3.98552400

2. 62133300

2.15416900

5.95388900

2.57263300

5.05359000

4.32958100

1.87191200

$-0.36482000$

$-1.39866400$ 


\begin{tabular}{|c|c|c|c|}
\hline \multicolumn{4}{|c|}{ Coordinates (from last standard orientation } \\
\hline ATOM & X & 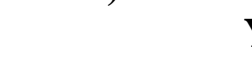 & \\
\hline \multicolumn{4}{|l|}{11} \\
\hline $\mathrm{C}$ & -2.02002800 & 3.19517300 & -0.25592100 \\
\hline $\mathrm{C}$ & -0.67982100 & 2.88121700 & -0.03701600 \\
\hline $\mathrm{C}$ & -0.26648400 & 1.54058900 & -0.00304800 \\
\hline C & -1.20972300 & 0.52224200 & -0.19472800 \\
\hline C & -2.55326100 & 0.83744600 & -0.42147100 \\
\hline $\mathrm{C}$ & -2.95876100 & 2.17323100 & -0.44969700 \\
\hline $\mathrm{H}$ & -2.33841900 & 4.23316300 & -0.27228000 \\
\hline $\mathrm{H}$ & -3.27709500 & 0.04376900 & -0.57841100 \\
\hline $\mathrm{H}$ & -4.00158800 & 2.42067000 & -0.62260000 \\
\hline $\mathrm{C}$ & 1.17335300 & 1.17710400 & 0.19306300 \\
\hline $\mathrm{H}$ & 1.34921100 & 0.09064700 & 0.20595500 \\
\hline $\mathrm{C}$ & 0.45733400 & 0.74034800 & 2.98236200 \\
\hline $\mathrm{C}$ & 0.65279600 & 0.80255700 & 4.35924200 \\
\hline $\mathrm{C}$ & 1.78948500 & 1.45231500 & 4.84749400 \\
\hline $\mathrm{C}$ & 2.69219000 & 2.01636900 & 3.94403100 \\
\hline $\mathrm{C}$ & 2.42756300 & 1.90720600 & 2.58033500 \\
\hline $\mathrm{N}$ & 1.33588000 & 1.28423700 & 2.12866000 \\
\hline $\mathrm{Na}$ & 3.03814200 & 2.77082500 & -1.91169700 \\
\hline 0 & 2.10267600 & 1.92466500 & -0.26294800 \\
\hline $\mathrm{H}$ & -0.89253200 & -0.51831600 & -0.18411200 \\
\hline $\mathrm{H}$ & 0.05045600 & 3.66933200 & 0.12294300 \\
\hline $\mathrm{H}$ & 3.58531000 & 2.52879700 & 4.28433400 \\
\hline $\mathrm{H}$ & 3.07813600 & 2.31430200 & 1.81148700 \\
\hline $\mathrm{H}$ & -0.41373000 & 0.26221300 & 2.54468300 \\
\hline $\mathrm{H}$ & 1.96749100 & 1.51812800 & 5.91614900 \\
\hline $\mathrm{H}$ & -0.07331900 & 0.35449000 & 5.02852800 \\
\hline
\end{tabular}




\begin{tabular}{lrrr}
\multicolumn{4}{l}{ 2,6-Lutidine attacking Benzaldehyde with Lithium ion } \\
Coordinates (from last standard orientation): \\
ATOM \\
I 1 & X & \\
C & -1.78722400 & 3.29781600 & -1.02579900 \\
C & -0.49610900 & 2.84181300 & -0.75555200 \\
C & -0.29702300 & 1.71417400 & 0.05180000 \\
C & -1.41247800 & 1.03811500 & 0.57015800 \\
C & -2.70273500 & 1.49597600 & 0.30687900 \\
C & -2.89222800 & 2.62913400 & -0.49135400 \\
H & -1.93194300 & 4.16978700 & -1.65617900 \\
H & 0.36236100 & 3.35521200 & -1.17556100 \\
H & -1.27452900 & 0.15570400 & 1.18923100 \\
H & -3.55853700 & 0.96877200 & 0.71661600 \\
H & -3.89655400 & 2.98326900 & -0.70202000 \\
C & 1.08105600 & 1.16235400 & 0.26806200 \\
H & 1.09665400 & 0.07295700 & 0.27028000 \\
C & 1.52737600 & 0.03064200 & 2.86832200 \\
C & 1.77186100 & 0.03282100 & 4.24389500 \\
C & 1.81426800 & 1.23680400 & 4.94023400 \\
C & 1.61442500 & 2.42007400 & 4.24005900 \\
C & 1.36442300 & 2.38538700 & 2.86404800 \\
N & 1.32060700 & 1.19949000 & 2.20612500 \\
H & 2.00445000 & 1.25064700 & 6.00884100 \\
H & 1.93043800 & -0.91070400 & 4.75393900 \\
H & 1.64498600 & 3.37944600 & 4.74467800 \\
O & 2.10186900 & 1.74785700 & -0.24902100 \\
Li & 3.47553200 & 2.11544600 & -1.15969500 \\
C & 1.12593200 & 3.67411600 & 2.12385900 \\
H & 0.06059300 & 3.80920700 & 1.91342100 \\
H & 1.45737300 & 4.51592000 & 2.73464900 \\
H & 1.65423000 & 3.69630800 & 1.17068600 \\
C & 1.50600800 & -1.28833300 & 2.13113400 \\
H & 0.53763700 & -1.48858100 & 1.66233200 \\
H & 2.27572500 & -1.33534900 & 1.35381400 \\
H & 1.70147700 & -2.10013400 & 2.83365000
\end{tabular}




\begin{tabular}{|c|c|c|c|}
\hline \multicolumn{4}{|c|}{ Coordinates (from last standard orientation): } \\
\hline ATOM & $\mathbf{X}$ & & \\
\hline \multicolumn{4}{|l|}{11} \\
\hline $\mathrm{C}$ & -2.21886000 & 3.09018600 & -0.38707000 \\
\hline C & -0.84256000 & 2.92785900 & -0.25852100 \\
\hline $\mathrm{C}$ & -0.29350700 & 1.63805200 & -0.15800900 \\
\hline $\mathrm{C}$ & -1.13979200 & 0.51696500 & -0.18756900 \\
\hline C & -2.51887400 & 0.68187100 & -0.32555700 \\
\hline $\mathrm{C}$ & -3.05760900 & 1.96763300 & -0.42348600 \\
\hline $\mathrm{H}$ & -2.64394100 & 4.08634100 & -0.45952900 \\
\hline $\mathrm{H}$ & -0.18864300 & 3.79340400 & -0.22255800 \\
\hline $\mathrm{H}$ & -0.71558300 & -0.48163500 & -0.11692000 \\
\hline $\mathrm{H}$ & -3.16890900 & -0.18654600 & -0.36015300 \\
\hline $\mathrm{H}$ & -4.13017200 & 2.09828500 & -0.52949000 \\
\hline C & 1.15775000 & 1.41874600 & -0.06697000 \\
\hline $\mathrm{H}$ & 1.47983000 & 0.37362900 & -0.01520100 \\
\hline C & 0.38597700 & 1.03843500 & 3.00745500 \\
\hline $\mathrm{C}$ & 0.57259000 & 0.99316600 & 4.39482700 \\
\hline $\mathrm{C}$ & 1.84374300 & 1.33303100 & 4.87283300 \\
\hline $\mathrm{C}$ & 2.87414600 & 1.70122700 & 3.99866100 \\
\hline C & 2.56665600 & 1.70992200 & 2.63341100 \\
\hline $\mathrm{N}$ & 1.35952600 & 1.38758500 & 2.16076200 \\
\hline 0 & 2.00757600 & 2.28789300 & -0.41279300 \\
\hline $\mathrm{Li}$ & 3.04066200 & 3.35285000 & -1.24958700 \\
\hline $\mathrm{C}$ & 4.24899600 & 2.06668900 & 4.49925800 \\
\hline $\mathrm{H}$ & 4.20697700 & 2.92886600 & 5.17342200 \\
\hline $\mathrm{H}$ & 4.69752500 & 1.23820500 & 5.05762500 \\
\hline $\mathrm{H}$ & 4.92228000 & 2.31680300 & 3.67489300 \\
\hline $\mathrm{C}$ & -0.54769300 & 0.59756900 & 5.32347600 \\
\hline $\mathrm{H}$ & -1.46263800 & 0.36834600 & 4.77140800 \\
\hline $\mathrm{H}$ & -0.27764000 & -0.28704100 & 5.91004700 \\
\hline $\mathrm{H}$ & -0.77422700 & 1.40192200 & 6.03142300 \\
\hline $\mathrm{H}$ & 3.31487700 & 1.98290000 & 1.89163600 \\
\hline $\mathrm{H}$ & -0.57648500 & 0.79270700 & 2.56487300 \\
\hline $\mathrm{H}$ & 2.03501900 & 1.31040800 & 5.94374300 \\
\hline
\end{tabular}




\section{$\underline{\text { 2,4,6-Collidine attacking Benzaldehyde with Lithium ion }}$}

\begin{tabular}{|c|c|c|c|}
\hline \multicolumn{4}{|c|}{ ATOM $\quad \mathrm{X}$} \\
\hline 11 & & & \\
\hline $\mathrm{C}$ & -1.31753400 & 3.74564500 & -0.47421600 \\
\hline $\mathrm{C}$ & -0.21680600 & 2.96540600 & -0.12418200 \\
\hline $\mathrm{C}$ & -0.34054200 & 1.57178100 & -0.01591700 \\
\hline $\mathrm{C}$ & -1.58019200 & 0.97454200 & -0.28958500 \\
\hline $\mathrm{C}$ & -2.67984900 & 1.75468000 & -0.65735400 \\
\hline $\mathrm{C}$ & -2.55183400 & 3.14212100 & -0.74201000 \\
\hline $\mathrm{H}$ & -1.21506600 & 4.82445800 & -0.54020000 \\
\hline $\mathrm{H}$ & 0.73522000 & 3.44502500 & 0.07457200 \\
\hline $\mathrm{H}$ & -1.68499300 & -0.10621100 & -0.23724500 \\
\hline $\mathrm{H}$ & -3.63069700 & 1.27888500 & -0.87611000 \\
\hline $\mathrm{H}$ & -3.40630200 & 3.75201500 & -1.01820900 \\
\hline $\mathrm{C}$ & 0.83000400 & 0.67409000 & 0.25843100 \\
\hline $\mathrm{H}$ & 0.54904500 & -0.35548900 & 0.49430700 \\
\hline $\mathrm{C}$ & 0.49070000 & 0.40676500 & 3.12029700 \\
\hline $\mathrm{C}$ & 0.73391500 & 0.61445200 & 4.47847100 \\
\hline $\mathrm{C}$ & 1.75927000 & 1.45929300 & 4.90767100 \\
\hline $\mathrm{C}$ & 2.52834100 & 2.05938300 & 3.90866400 \\
\hline $\mathrm{C}$ & 2.26907800 & 1.83128900 & 2.55571900 \\
\hline $\mathrm{N}$ & 1.24748800 & 1.02245400 & 2.17318700 \\
\hline 0 & 1.90774400 & 0.82993600 & -0.41533900 \\
\hline $\mathrm{Li}$ & 2.87548600 & 0.85622700 & -1.80610200 \\
\hline $\mathrm{C}$ & -0.62598400 & -0.52422700 & 2.72028300 \\
\hline $\mathrm{H}$ & -0.25748700 & -1.38293300 & 2.14913300 \\
\hline $\mathrm{H}$ & -1.11525500 & -0.91409800 & 3.61409500 \\
\hline $\mathrm{H}$ & -1.38209000 & -0.01351600 & 2.12015600 \\
\hline $\mathrm{C}$ & 3.17732400 & 2.48429700 & 1.54151000 \\
\hline $\mathrm{H}$ & 2.62760200 & 2.99253700 & 0.75024500 \\
\hline $\mathrm{H}$ & 3.81198400 & 3.21548600 & 2.04520800 \\
\hline $\mathrm{H}$ & 3.82544800 & 1.73943200 & 1.07021500 \\
\hline $\mathrm{C}$ & 2.00970000 & 1.72248800 & 6.36754100 \\
\hline $\mathrm{H}$ & 1.75415500 & 0.85352500 & 6.97962900 \\
\hline $\mathrm{H}$ & 3.05362700 & 1.98692200 & 6.55412900 \\
\hline $\mathrm{H}$ & 1.39008800 & 2.55991200 & 6.71108000 \\
\hline $\mathrm{H}$ & 3.35126400 & 2.71491500 & 4.17500500 \\
\hline $\mathrm{H}$ & 0.10773600 & 0.10328000 & 5.20246600 \\
\hline
\end{tabular}




\begin{tabular}{|c|c|c|c|}
\hline \multicolumn{4}{|c|}{ Coordinates (from last standard orientation): } \\
\hline ATOM & $Y$ & 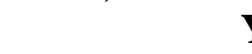 & \\
\hline \multicolumn{4}{|l|}{11} \\
\hline $\mathrm{C}$ & -2.57042000 & 3.02439400 & -0.32296800 \\
\hline $\mathrm{C}$ & -1.17868900 & 2.97470500 & -0.32452300 \\
\hline $\mathrm{C}$ & -0.51975100 & 1.75116200 & -0.52833600 \\
\hline $\mathrm{C}$ & -1.26839700 & 0.58050400 & -0.72124600 \\
\hline $\mathrm{C}$ & -2.66390100 & 0.63324800 & -0.72559600 \\
\hline $\mathrm{C}$ & -3.31373600 & 1.85388600 & -0.52664400 \\
\hline $\mathrm{H}$ & -3.08027900 & 3.96816500 & -0.15658200 \\
\hline $\mathrm{H}$ & -0.59914900 & 3.87510400 & -0.14131500 \\
\hline $\mathrm{H}$ & -0.76008000 & -0.36752300 & -0.87966200 \\
\hline $\mathrm{H}$ & -3.24022300 & -0.27239500 & -0.88522700 \\
\hline $\mathrm{H}$ & -4.39851500 & 1.89614000 & -0.52673900 \\
\hline $\mathrm{C}$ & 0.95732600 & 1.66463900 & -0.58097900 \\
\hline $\mathrm{H}$ & 1.35443400 & 0.65895000 & -0.75544200 \\
\hline $\mathrm{C}$ & 0.84643400 & 0.87974600 & 2.47829400 \\
\hline $\mathrm{N}$ & 1.39087700 & 1.45879400 & 1.40586600 \\
\hline 0 & 1.68422100 & 2.65547300 & -0.92769300 \\
\hline $\mathrm{Li}$ & 1.96338700 & 3.92192000 & -2.05489200 \\
\hline $\mathrm{H}$ & -0.13968600 & 0.44148000 & 2.41784300 \\
\hline $\mathrm{C}$ & 2.84210800 & 1.62826700 & 3.09467800 \\
\hline $\mathrm{H}$ & 3.76512400 & 1.92041000 & 3.57373500 \\
\hline $\mathrm{C}$ & 1.72565000 & 0.96349600 & 3.57581300 \\
\hline $\mathrm{H}$ & 1.56665200 & 0.59491200 & 4.57764700 \\
\hline $\mathrm{N}$ & 2.60184200 & 1.90284100 & 1.78925200 \\
\hline $\mathrm{H}$ & 3.17987000 & 2.38594000 & 1.11530000 \\
\hline
\end{tabular}




\section{ENERGY MINIMA FOR PRODUCTS}

\section{Pyridine-Benzaldehyde with Lithium ion Product}

\section{Coordinates (from last standard orientation):}

\section{ATOM}

11

C

C

C

$\mathrm{C}$

C

C

$\mathrm{H}$

$\mathrm{H}$

$\mathrm{H}$

$\mathrm{H}$

$\mathrm{H}$

C

$\mathrm{H}$

C

C

C

C

C

N

$\mathrm{H}$

$\mathrm{H}$

$\mathrm{H}$

$\mathrm{H}$

$\mathrm{H}$

O

Li
X

$-1.95953200$

$-0.66029500$

$-0.22090200$

$-1.09498600$

$-2.39684300$

$-2.83007900$

$-2.29658200$

0.01434900

$-0.75805000$

$-3.06586100$

$-3.84019000$

1.20791000

1.34783400

0.45743000

0.58798700

1.64876200

2.54815800

2.36807100

1.33949800

1.76968900

$-0.34805800$

$-0.13272700$

3. 38422800

3. 01528700

2.16169000

2.71350800
Y

Z

3.25153900

2.89900300

1.57431800

0.61310700

0.96775700

2. 28571800

4.27656100

3.65094200

$-0.41321300$

0.21699000

2.56280500

1.18712100

0.10267000

0.60878900

0.62714700

1.33759600

2.00402600

1.94327900

1.26074000

1.36803000

0.09098300

0.09608000

2.56329500

2. 41665900

1.93770500

2. 64954300
$-0.09755900$

0.26716800

0.12130600

$-0.39946000$

$-0.77117000$

$-0.61809600$

0.02400900

0.66827000

$-0.52898800$

$-1.18019900$

$-0.90324900$

0.46968800

0.31975700

2.87476200

4.25437200

4.82665900

3.99475400

2. 61677200

2.08999100

5.90466100

2.36707000

4.86546900

4.39879600

1.88853100

$-0.06332500$

$-1.49500900$ 


\begin{tabular}{|c|c|c|c|}
\hline \multicolumn{4}{|c|}{$\begin{array}{l}\text { Coordinates (from last standard orientation): } \\
\text {. }\end{array}$} \\
\hline ATOM & $\mathbf{X}$ & Y & \\
\hline \multicolumn{4}{|l|}{11} \\
\hline $\mathrm{C}$ & -1.95180800 & 3.26769800 & -0.11549000 \\
\hline $\mathrm{C}$ & -0.64663200 & 2.92363800 & 0.23627100 \\
\hline $\mathrm{C}$ & -0.20111500 & 1.60047300 & 0.09475700 \\
\hline $\mathrm{C}$ & -1.07732400 & 0.63231200 & -0.40955200 \\
\hline $\mathrm{C}$ & -2.38524500 & 0.97773500 & -0.76946900 \\
\hline $\mathrm{C}$ & -2.82380000 & 2.29441200 & -0.62009300 \\
\hline $\mathrm{H}$ & -2.29373200 & 4.29131900 & 0.00574800 \\
\hline $\mathrm{H}$ & 0.03048000 & 3.67849800 & 0.62752300 \\
\hline $\mathrm{H}$ & -0.73655100 & -0.39342500 & -0.53474000 \\
\hline $\mathrm{H}$ & -3.05539500 & 0.22080500 & -1.16540100 \\
\hline $\mathrm{H}$ & -3.83881200 & 2.56441500 & -0.89472000 \\
\hline $\mathrm{C}$ & 1.23422000 & 1.22494900 & 0.42745300 \\
\hline $\mathrm{H}$ & 1.38485000 & 0.13865500 & 0.28512600 \\
\hline $\mathrm{C}$ & 0.45375100 & 0.58049000 & 2.87363500 \\
\hline $\mathrm{C}$ & 0.57499000 & 0.57380100 & 4.25565200 \\
\hline $\mathrm{C}$ & 1.62517100 & 1.28471900 & 4.84630000 \\
\hline $\mathrm{C}$ & 2.52203400 & 1.97659600 & 4.03213500 \\
\hline $\mathrm{C}$ & 2.34747600 & 1.93803900 & 2.65182900 \\
\hline $\mathrm{N}$ & 1.33156500 & 1.25512400 & 2.10825200 \\
\hline $\mathrm{H}$ & 1.74005200 & 1.29629500 & 5.92539200 \\
\hline $\mathrm{H}$ & -0.34354100 & 0.06278400 & 2.35207900 \\
\hline $\mathrm{H}$ & -0.14306200 & 0.02433600 & 4.85366900 \\
\hline $\mathrm{H}$ & 3.34957100 & 2.53691700 & 4.45227400 \\
\hline $\mathrm{H}$ & 2.99033300 & 2.43138100 & 1.93084500 \\
\hline 0 & 2.17900700 & 1.99602700 & -0.04803000 \\
\hline $\mathrm{Na}$ & 2.81405700 & 2.85630800 & -1.81843500 \\
\hline
\end{tabular}




\begin{tabular}{lrrr}
\multicolumn{4}{l}{ 2,6-Lutidine-Benzaldehyde with Lithium ion Product } \\
Coordinates (from last standard orientation): \\
ATOM \\
I 1 & X & \\
C & -1.64279900 & 3.14647500 & -1.23580100 \\
C & -0.37070400 & 2.73344600 & -0.82937100 \\
C & -0.22233300 & 1.78264100 & 0.18424200 \\
C & -1.37045100 & 1.23434200 & 0.77478700 \\
C & -2.64218400 & 1.64756400 & 0.37388900 \\
C & -2.78153200 & 2.60731900 & -0.63340700 \\
H & -1.74320900 & 3.88710000 & -2.02349500 \\
H & 0.51398200 & 3.15226600 & -1.29674900 \\
H & -1.28212300 & 0.48530500 & 1.55819100 \\
H & -3.52205100 & 1.21882000 & 0.84377200 \\
H & -3.76982500 & 2.92715300 & -0.94845300 \\
C & 1.17246100 & 1.26385100 & 0.54969800 \\
H & 1.19083300 & 0.19997500 & 0.30029200 \\
C & 1.50986900 & -0.01041000 & 2.75891300 \\
C & 1.68147700 & -0.06835800 & 4.14067100 \\
C & 1.63844300 & 1.09263700 & 4.90528100 \\
C & 1.42422000 & 2.30656800 & 4.26540900 \\
C & 1.24508700 & 2.35892100 & 2.88277300 \\
N & 1.28716200 & 1.19963000 & 2.15698000 \\
H & 1.77376600 & 1.05038200 & 5.98133100 \\
H & 1.85218700 & -1.03280500 & 4.60420500 \\
H & 1.38758500 & 3.23332800 & 4.82620500 \\
O & 2.21947800 & 1.92067500 & 0.05653000 \\
Li & 3.50889300 & 2.34942900 & -0.90640400 \\
C & 0.99034900 & 3.68407900 & 2.22251200 \\
H & -0.07163200 & 3.79656900 & 1.98304300 \\
H & 1.27099300 & 4.48495900 & 2.90880500 \\
H & 1.55104200 & 3.78265100 & 1.29424600 \\
C & 1.57839000 & -1.28820800 & 1.95906500 \\
H & 0.64254500 & -1.50454000 & 1.43516500 \\
H & 2.38546800 & -1.26695600 & 1.22026900 \\
H & 1.77453000 & -2.11866100 & 2.63893600
\end{tabular}




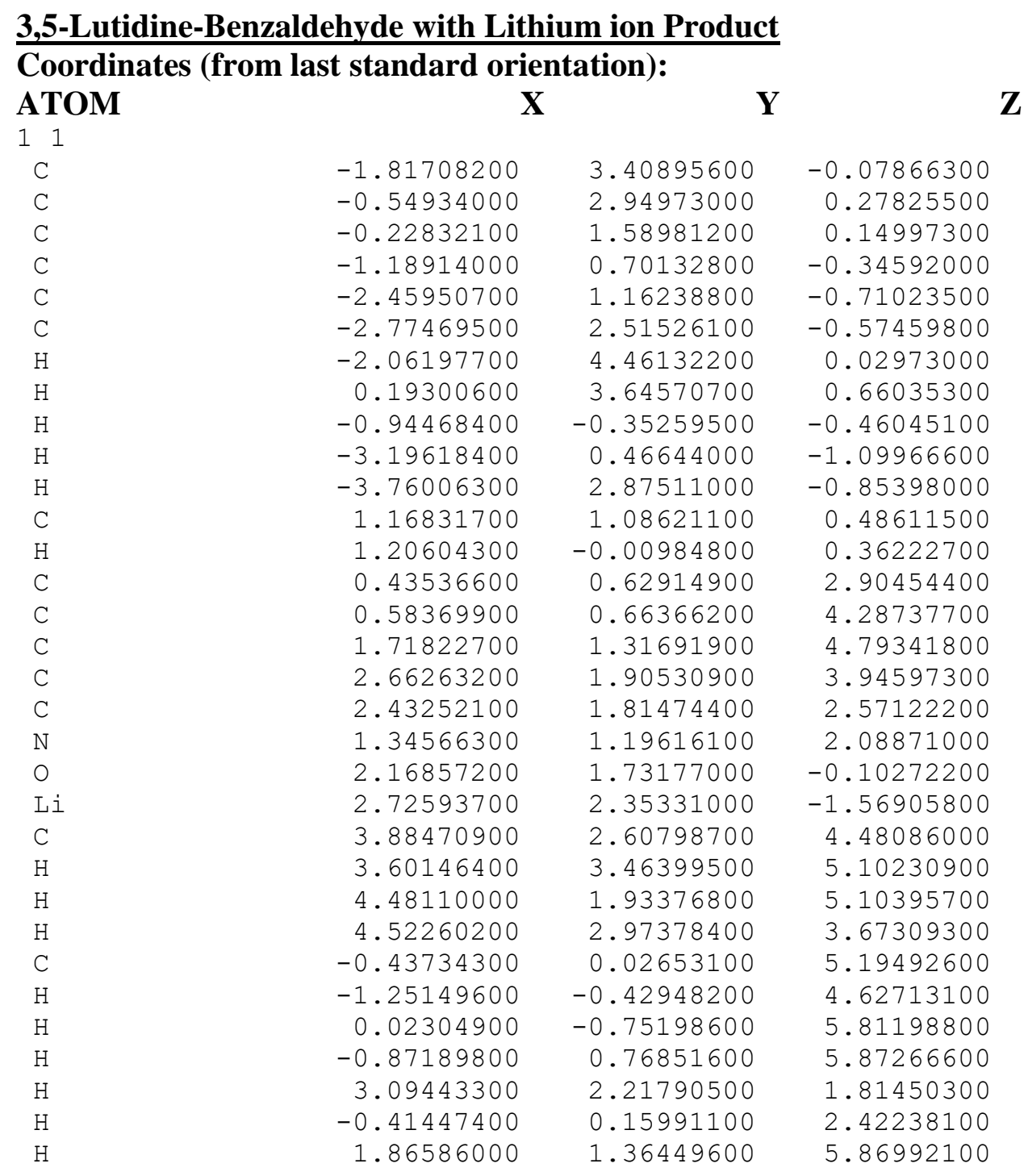




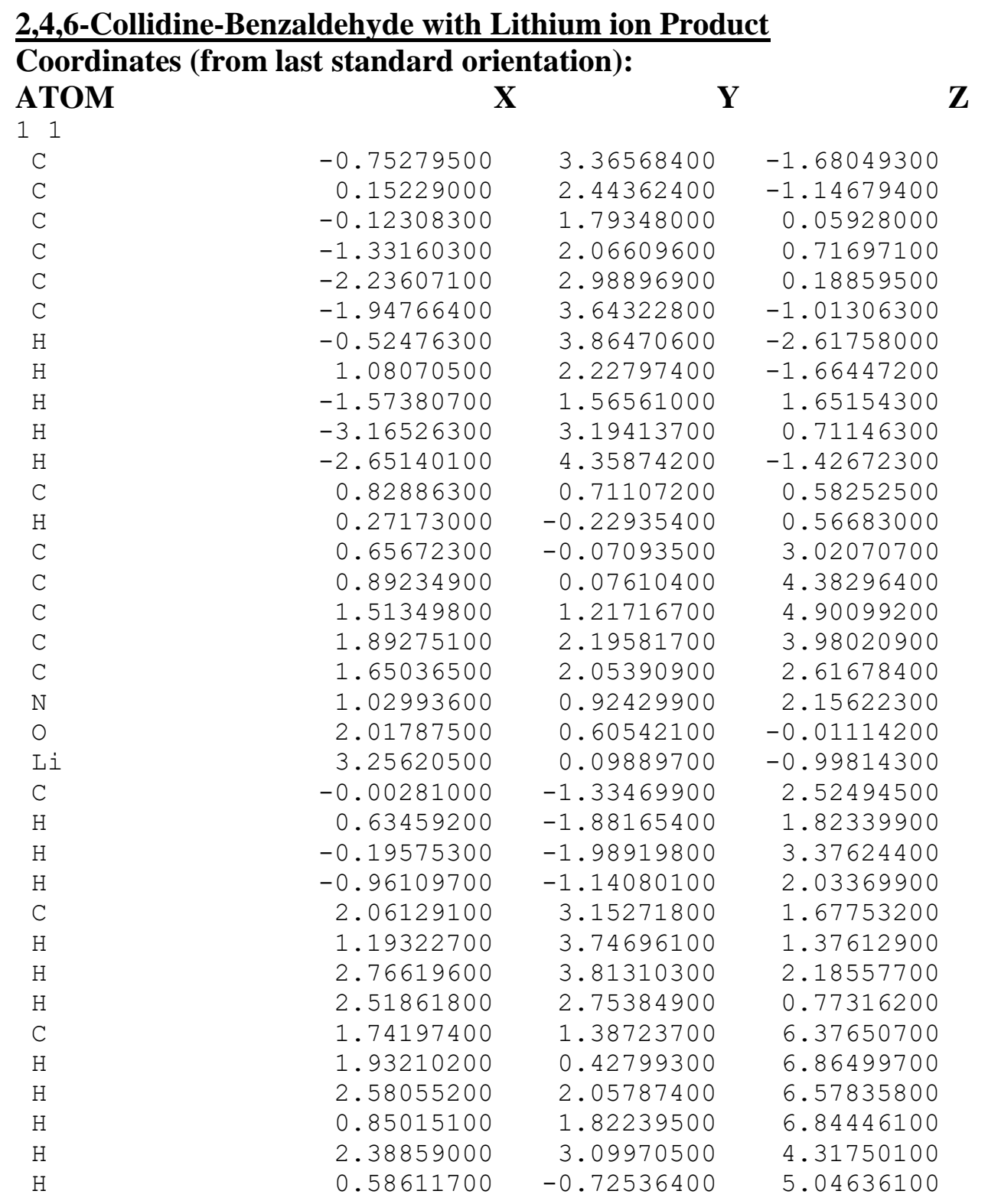




\begin{tabular}{lrrr}
\multicolumn{4}{l}{ Pyrazole-Benzaldehyde with Lithium ion Product } \\
Coordinates (from last standard orientation): \\
ATOM \\
I 1
\end{tabular}




\section{${ }^{1} \mathrm{H},{ }^{13} \mathrm{C}$, AND ${ }^{19} \mathrm{~F}$ NMR SPECTRA OF SYNTHESIZED COMPOUNDS}

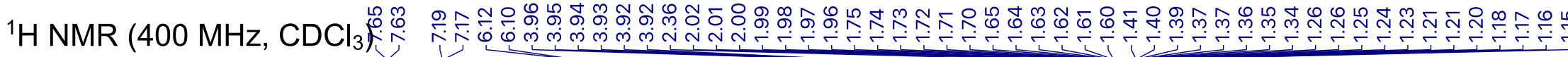
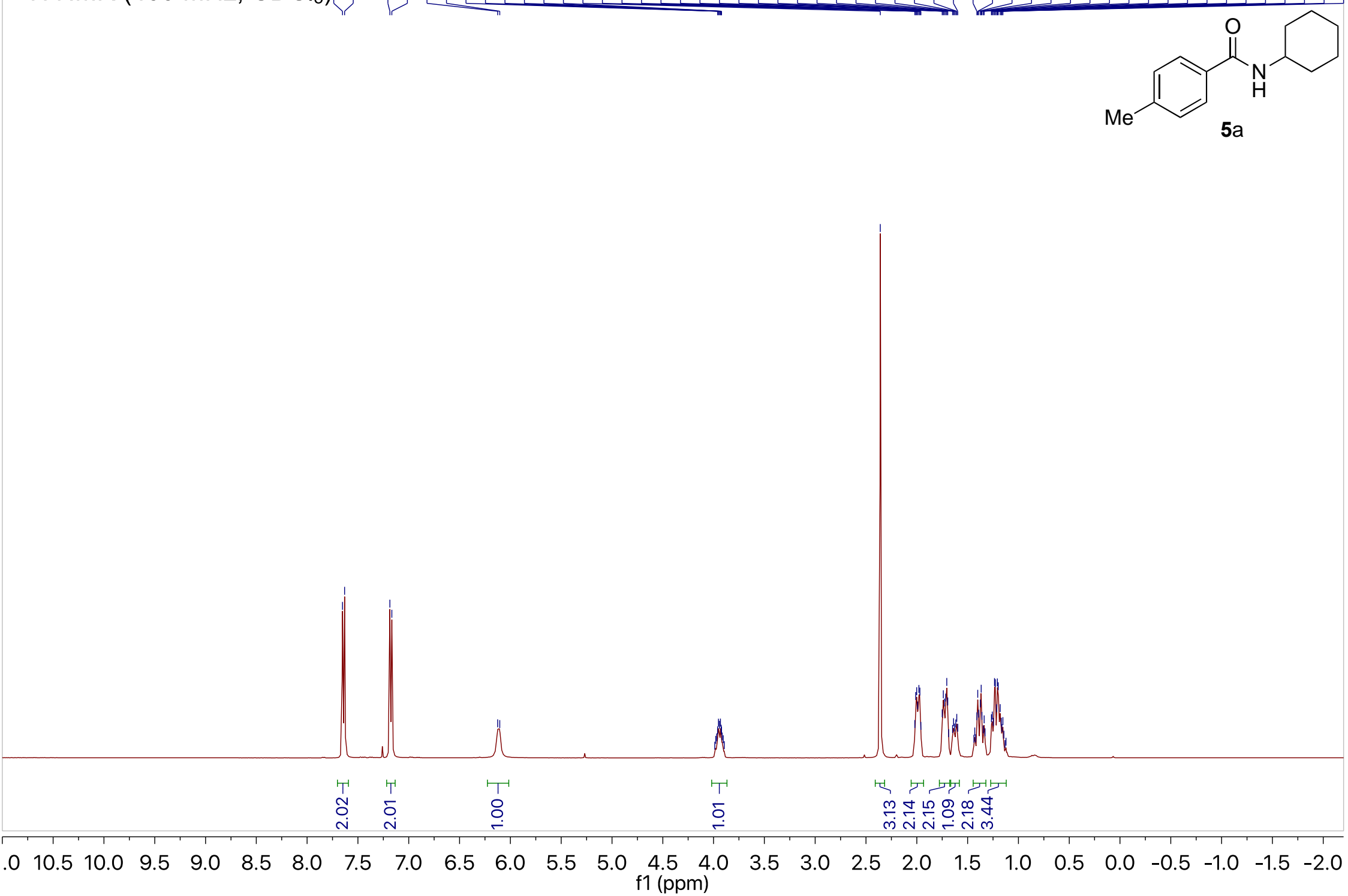


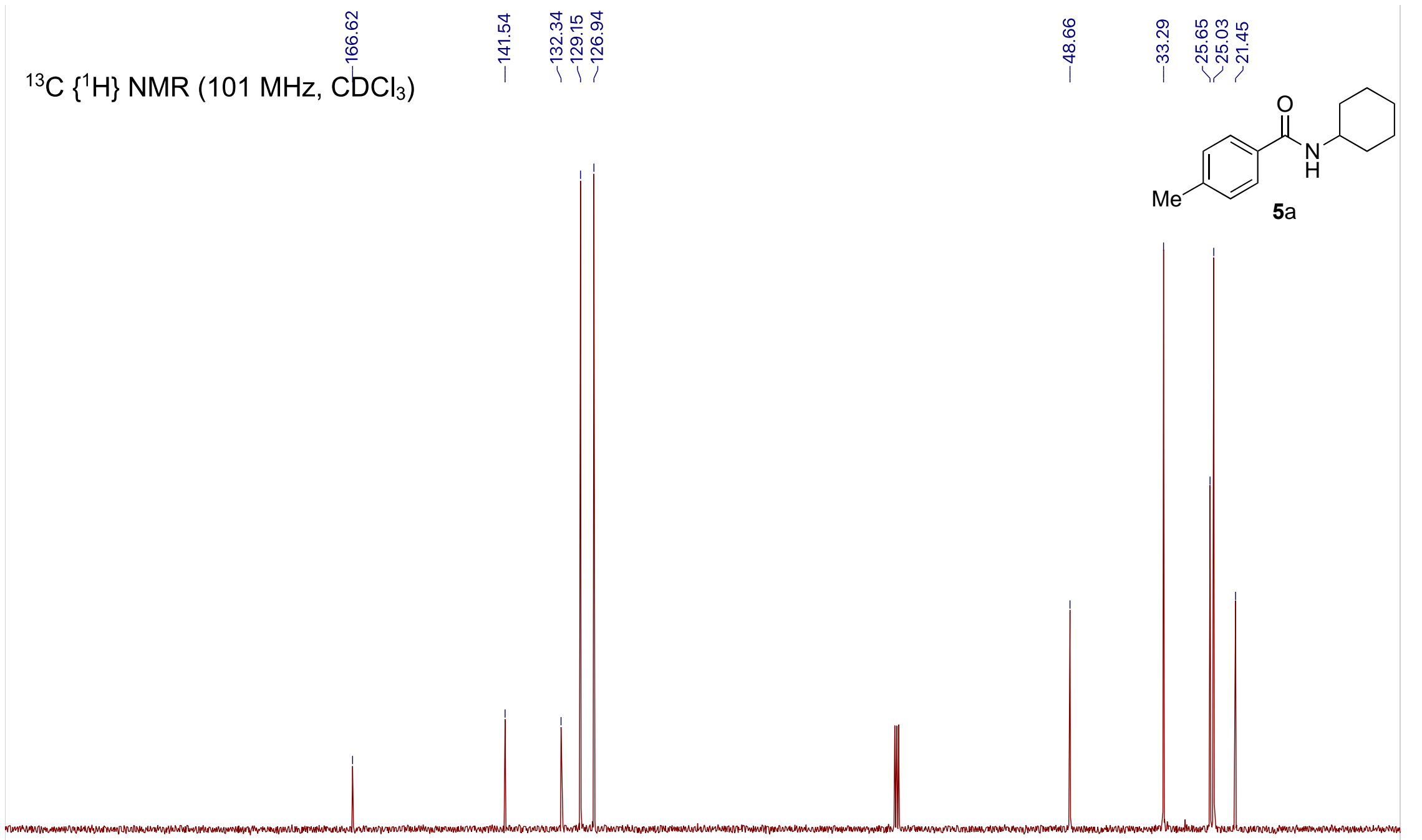

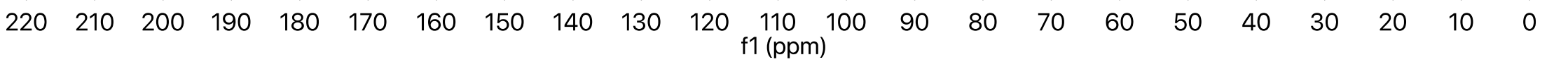




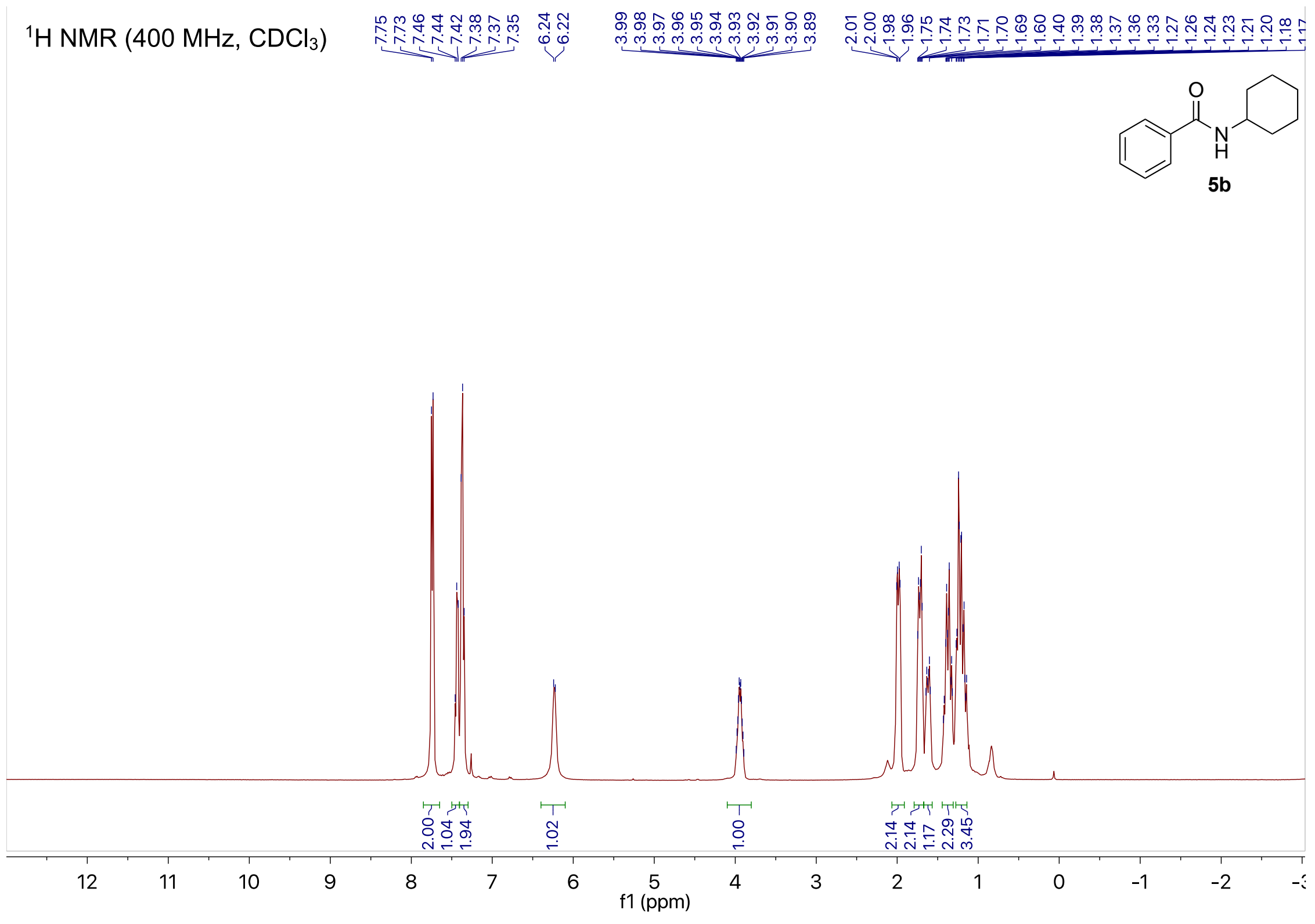




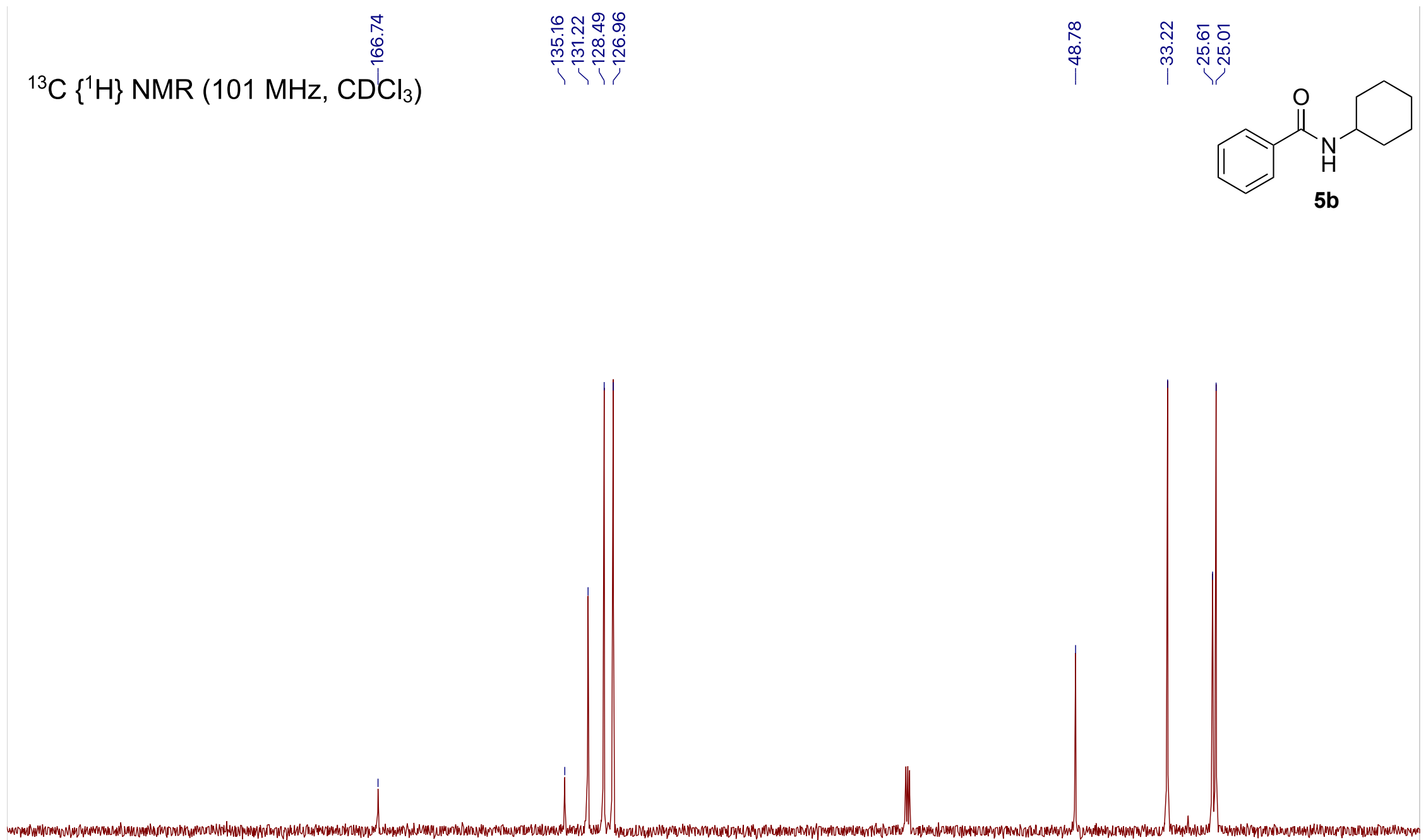

$\begin{array}{lllllllllllllllllllllll}220 & 210 & 200 & 190 & 180 & 170 & 160 & 150 & 140 & 130 & 120 \\ \mathrm{f} 1(\mathrm{ppm}) & 110 & 100 & 90 & 80 & 70 & 60 & 50 & 40 & 30 & 20 & 10 & 0\end{array}$ 


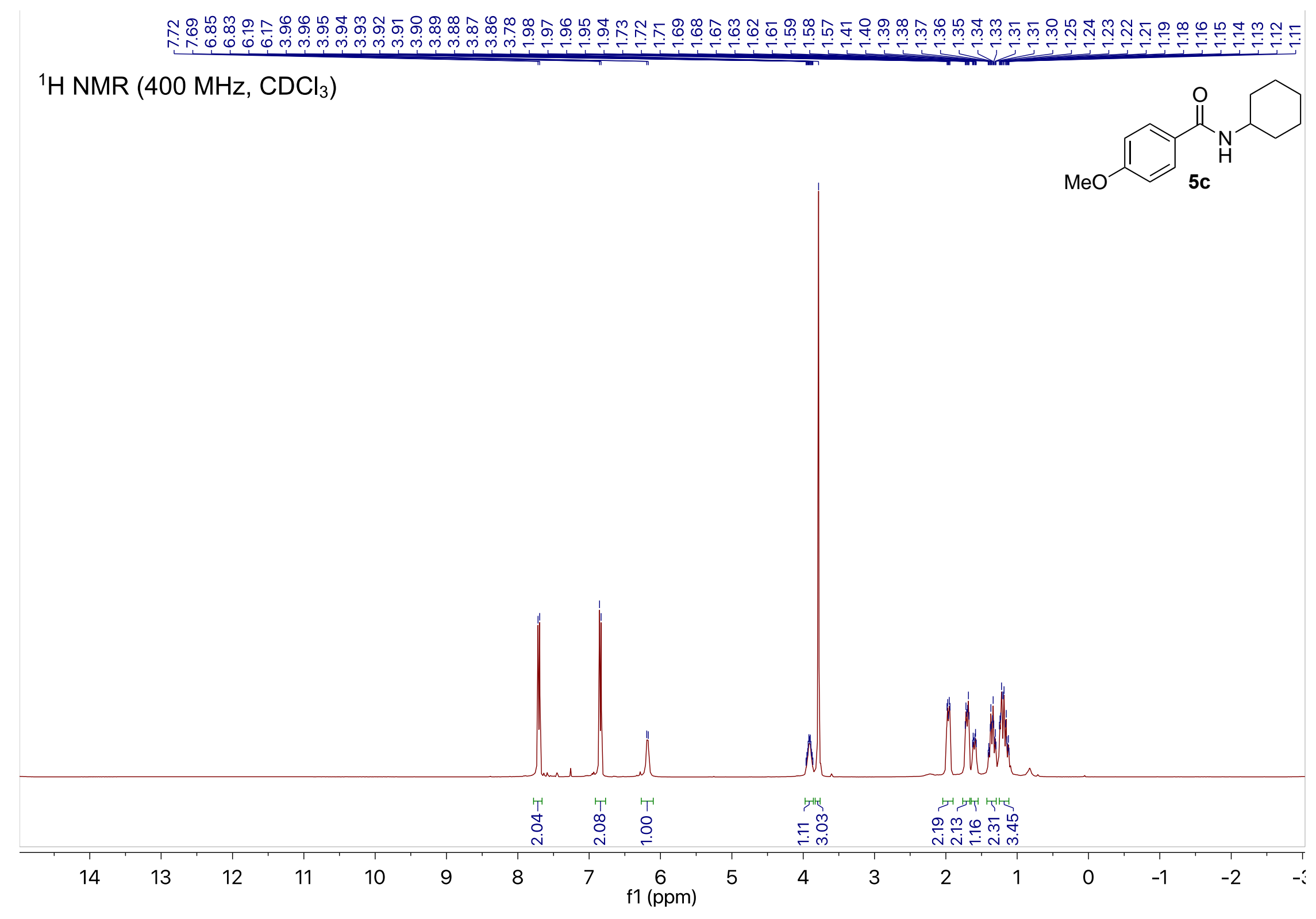




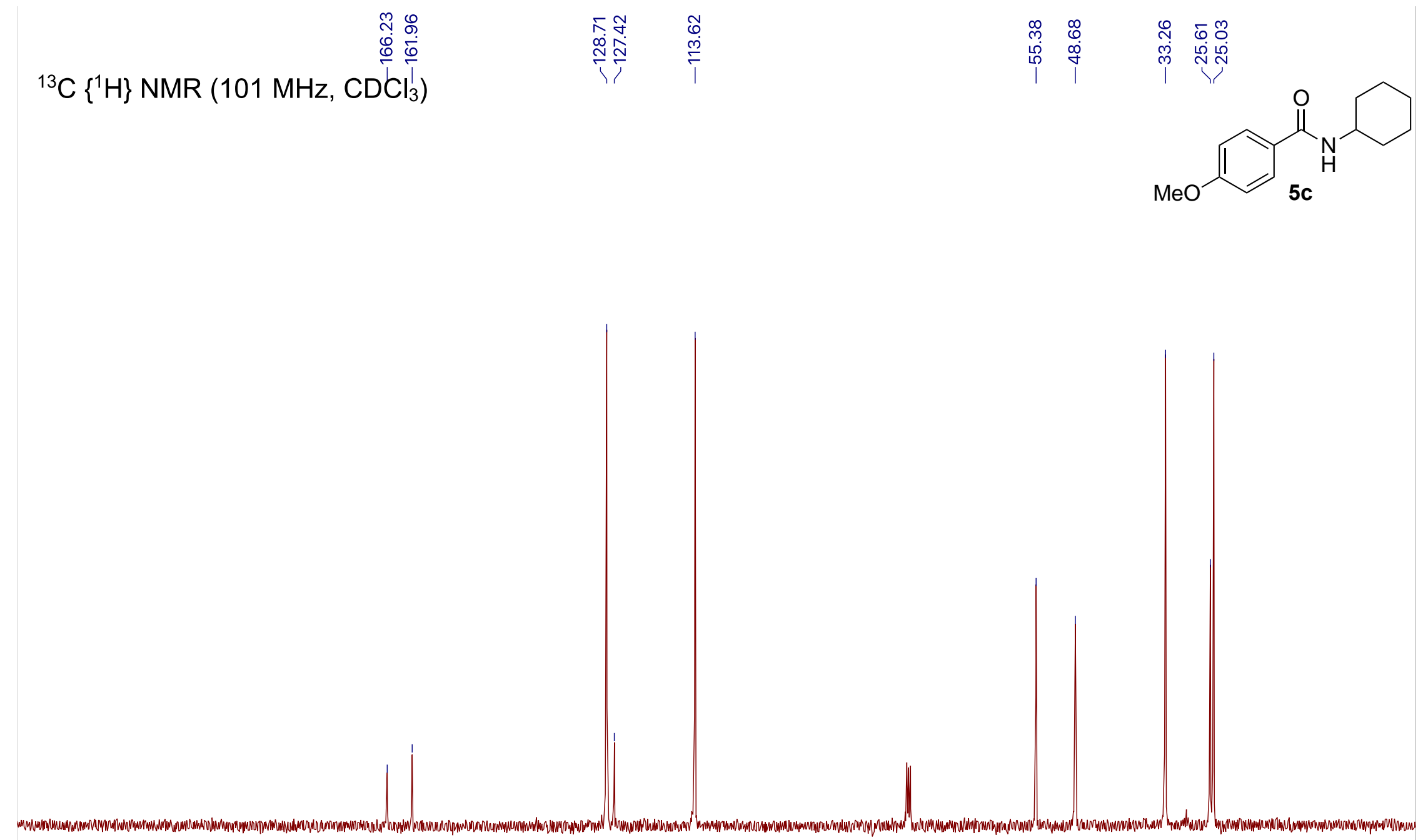

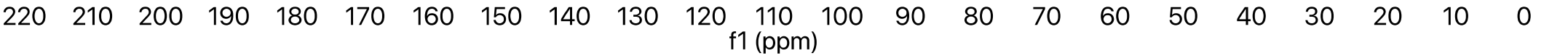




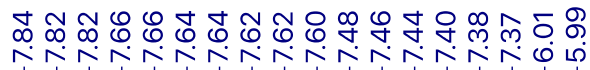

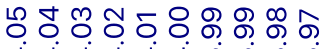

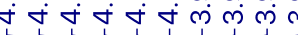

${ }^{1} \mathrm{H}$ NMR (400 MHz, $\mathrm{CDCl}_{3}$ )

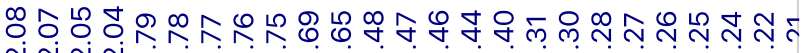
ninn

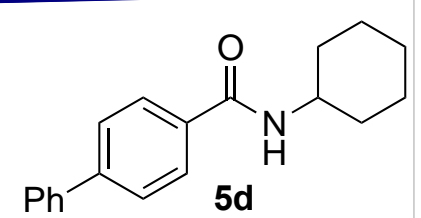

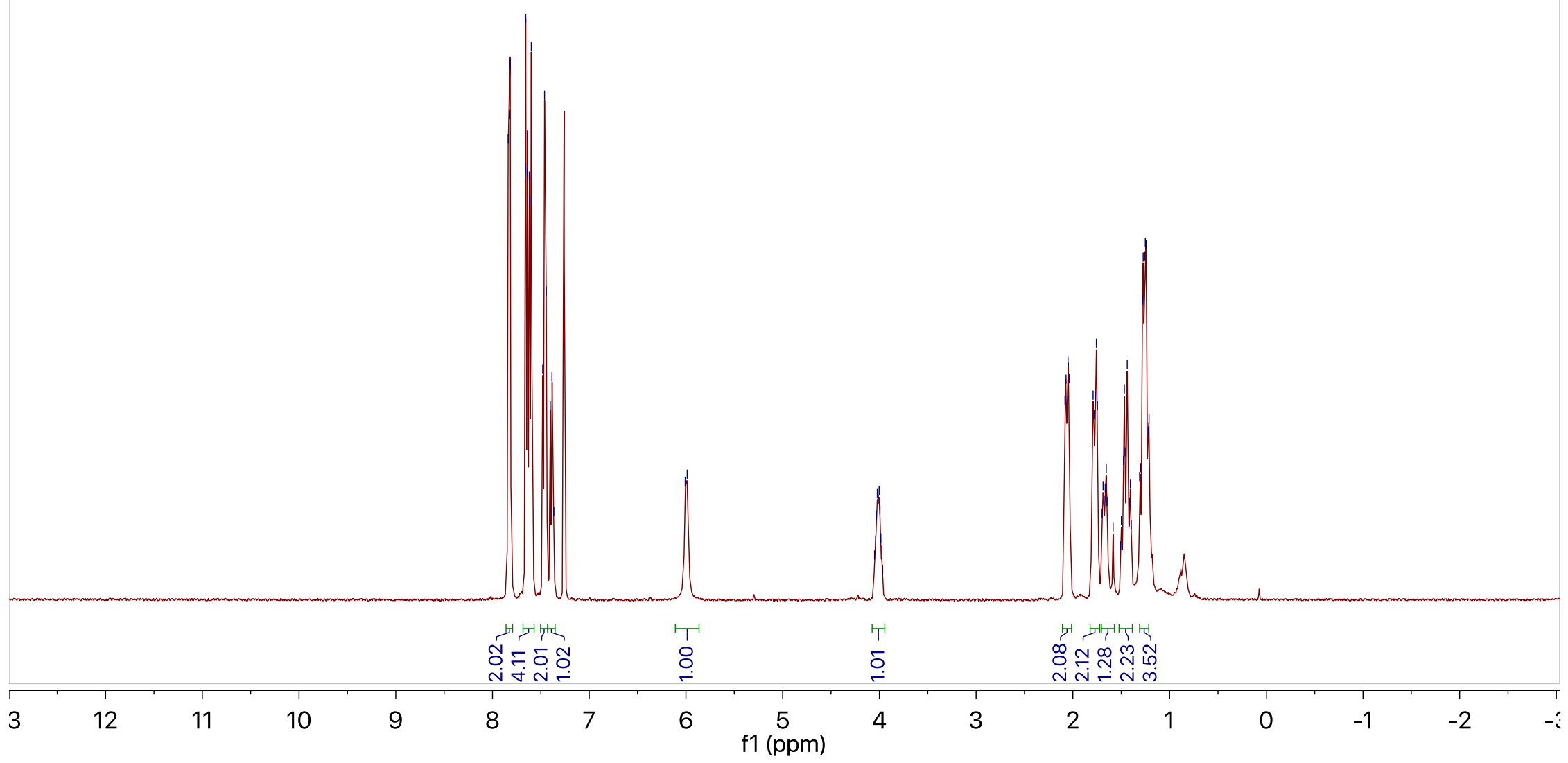



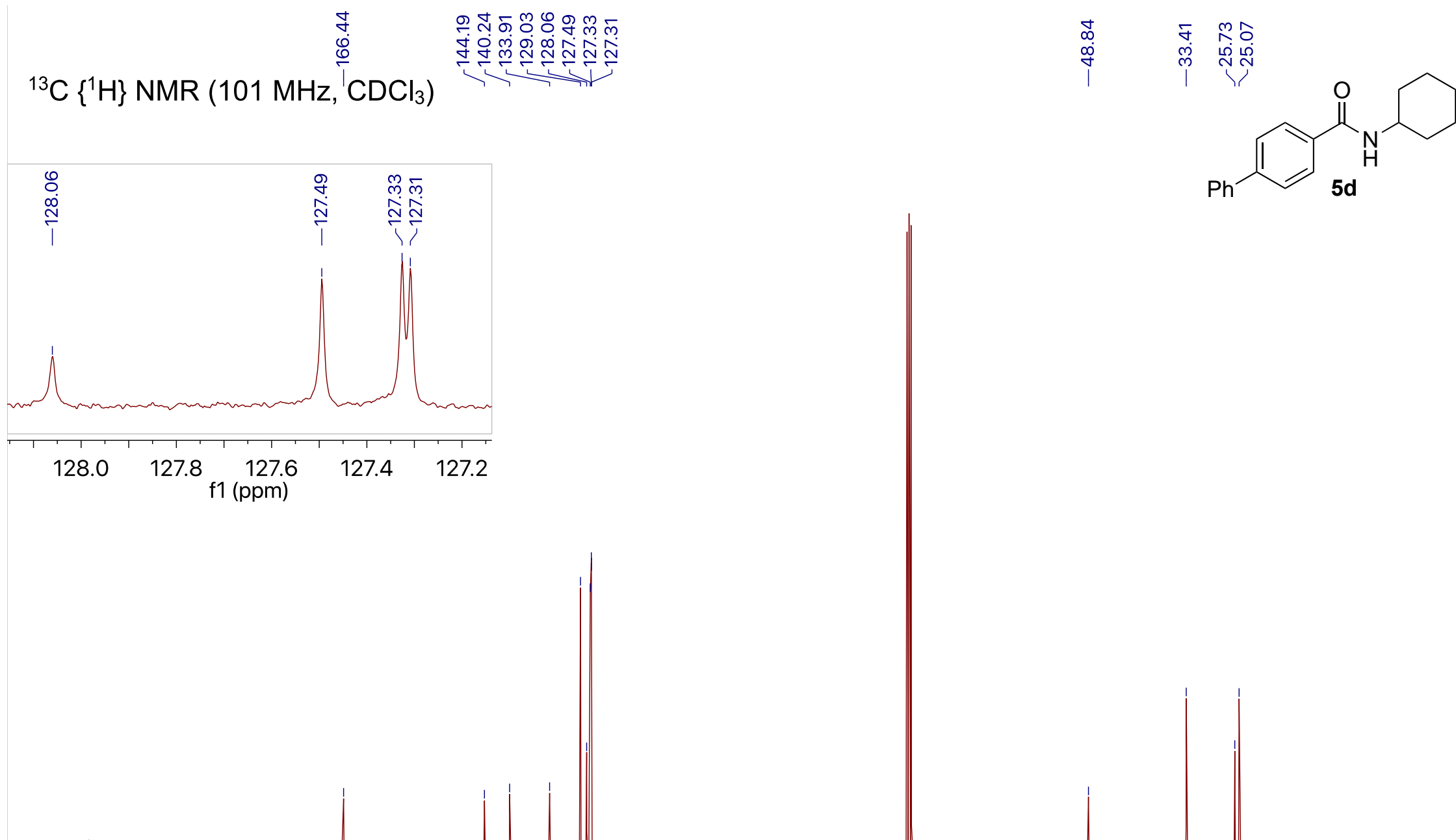

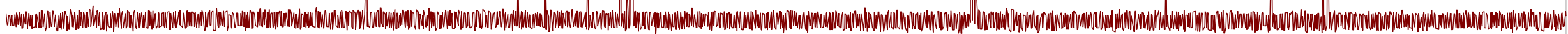

$\begin{array}{llllllllllllllllllllll}210 & 200 & 190 & 180 & 170 & 160 & 150 & 140 & 130 & 120 & \begin{array}{c}110 \\ \mathrm{f} 1(\mathrm{ppm})\end{array} & 90 & 80 & 70 & 60 & 50 & 40 & 30 & 20 & 10 & 0\end{array}$




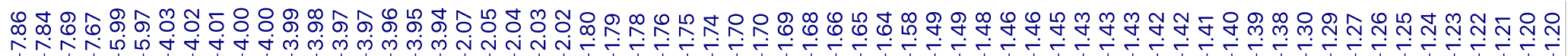
${ }^{1} \mathrm{H}$ NMR $\left(400 \mathrm{MHz}, \mathrm{CDCl}_{3}\right)$
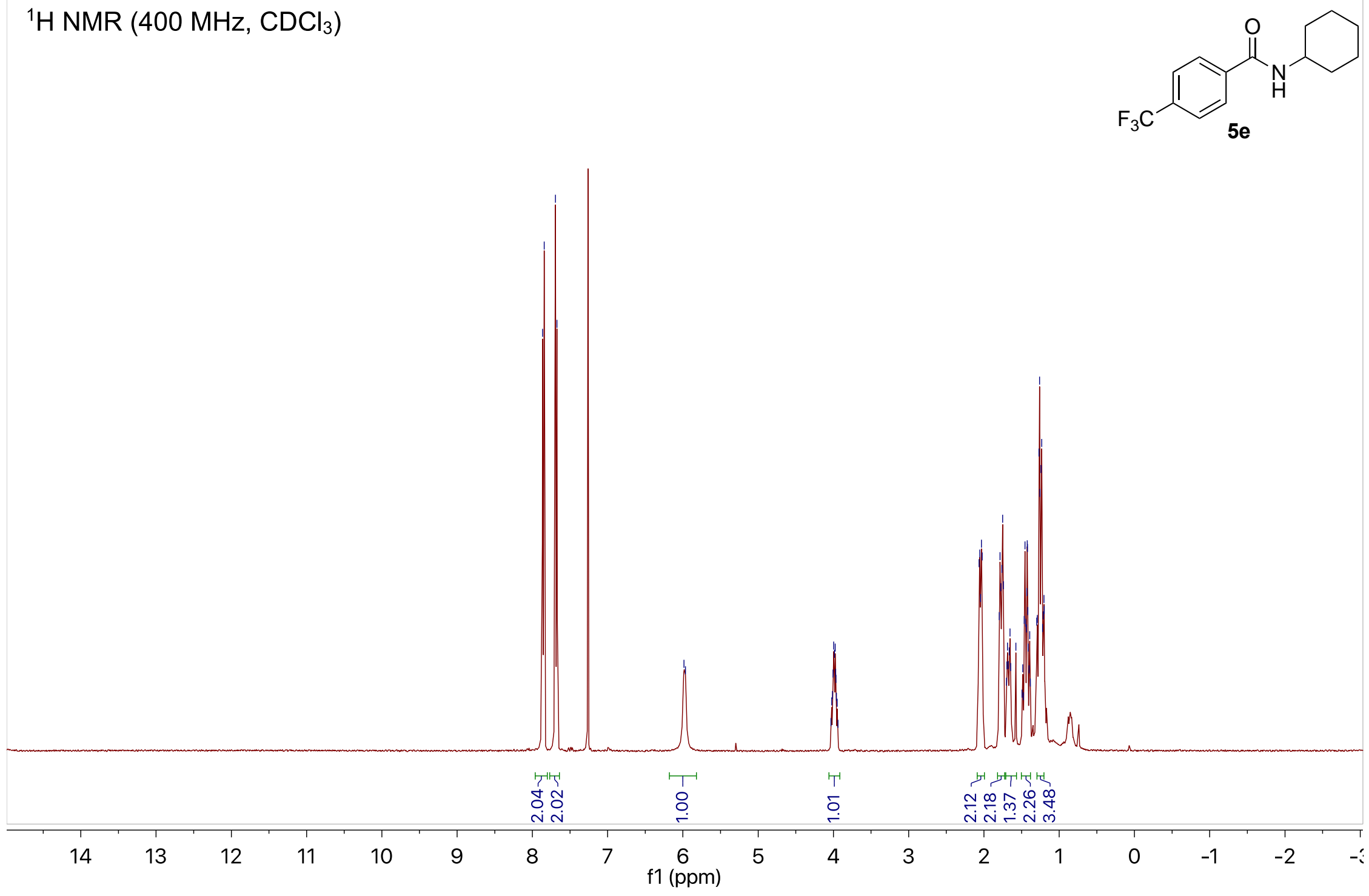


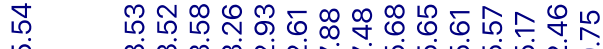

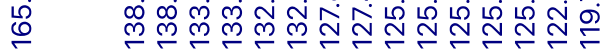
${ }^{13} \mathrm{C}\left\{{ }^{1} \mathrm{H}\right\}$ NMR $\left(101 \mathrm{MHz}, \mathrm{CDCl}_{3}\right)$

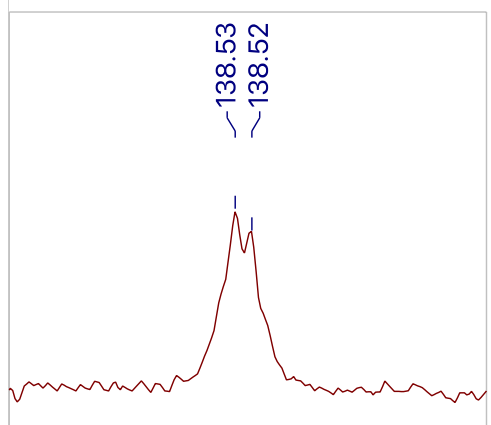

$\begin{array}{lllll}8.7 & 138.6 & 138.5 & 138.4\end{array}$

f1 (ppm)
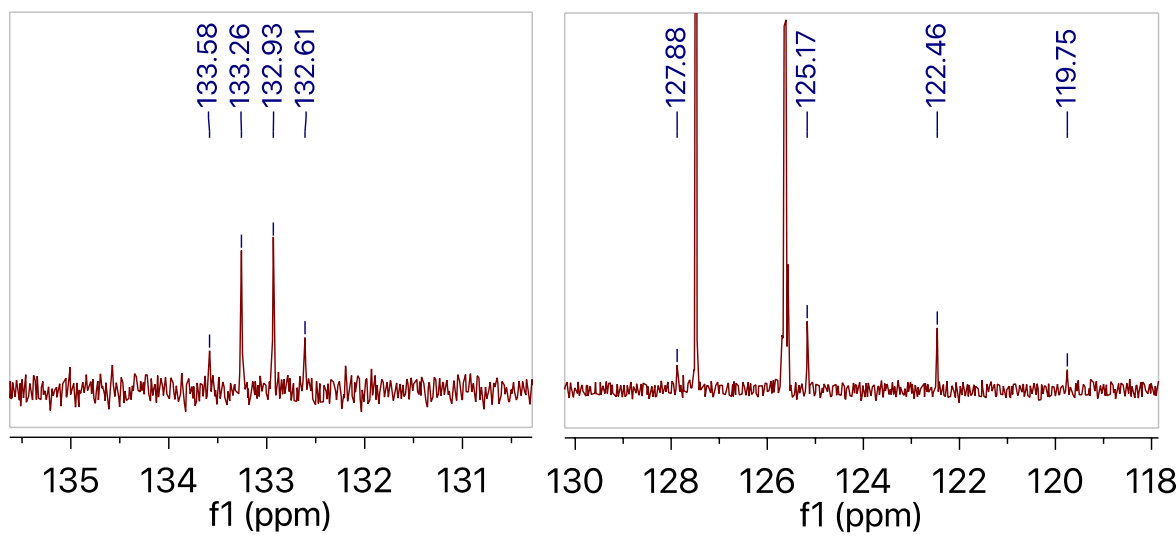

$\stackrel{0}{\stackrel{9}{\zeta}}$
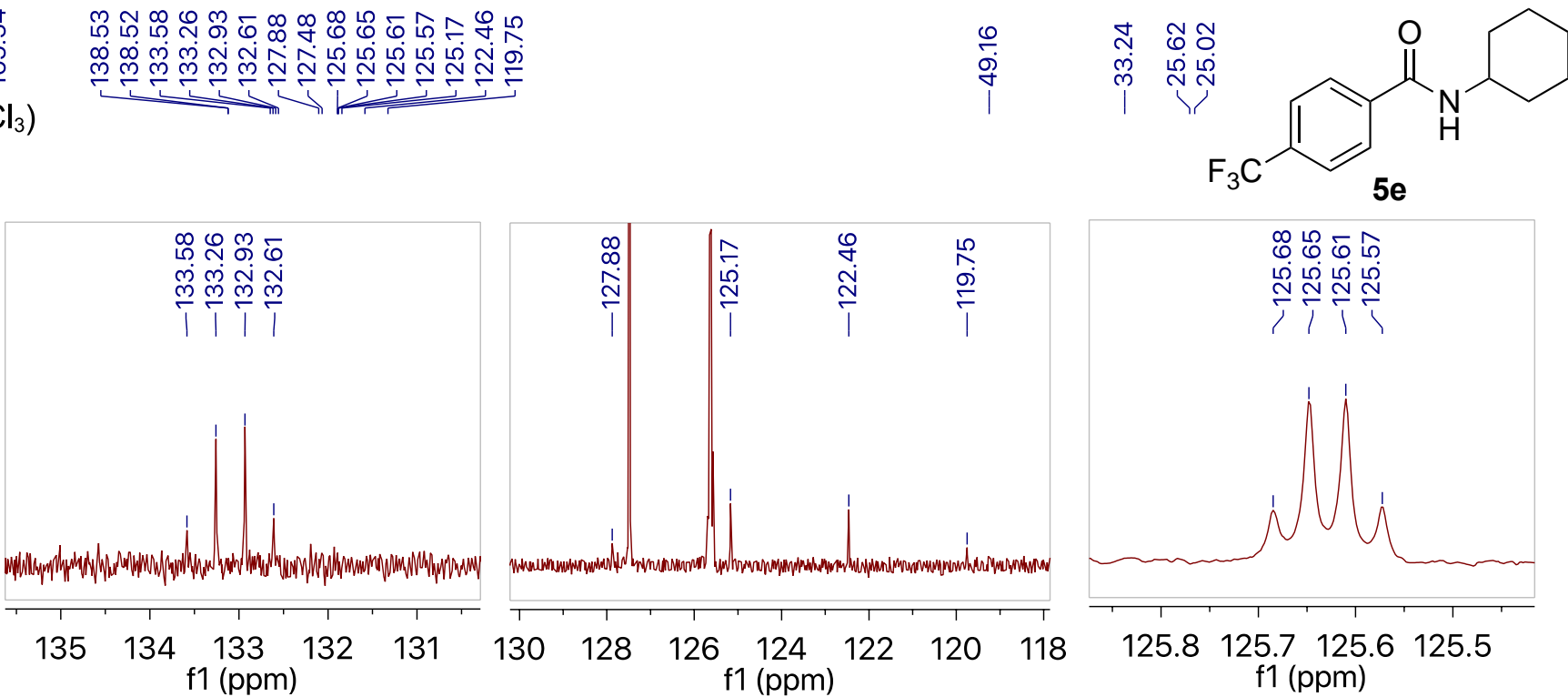

f1 (ppm)

f1 (ppm)
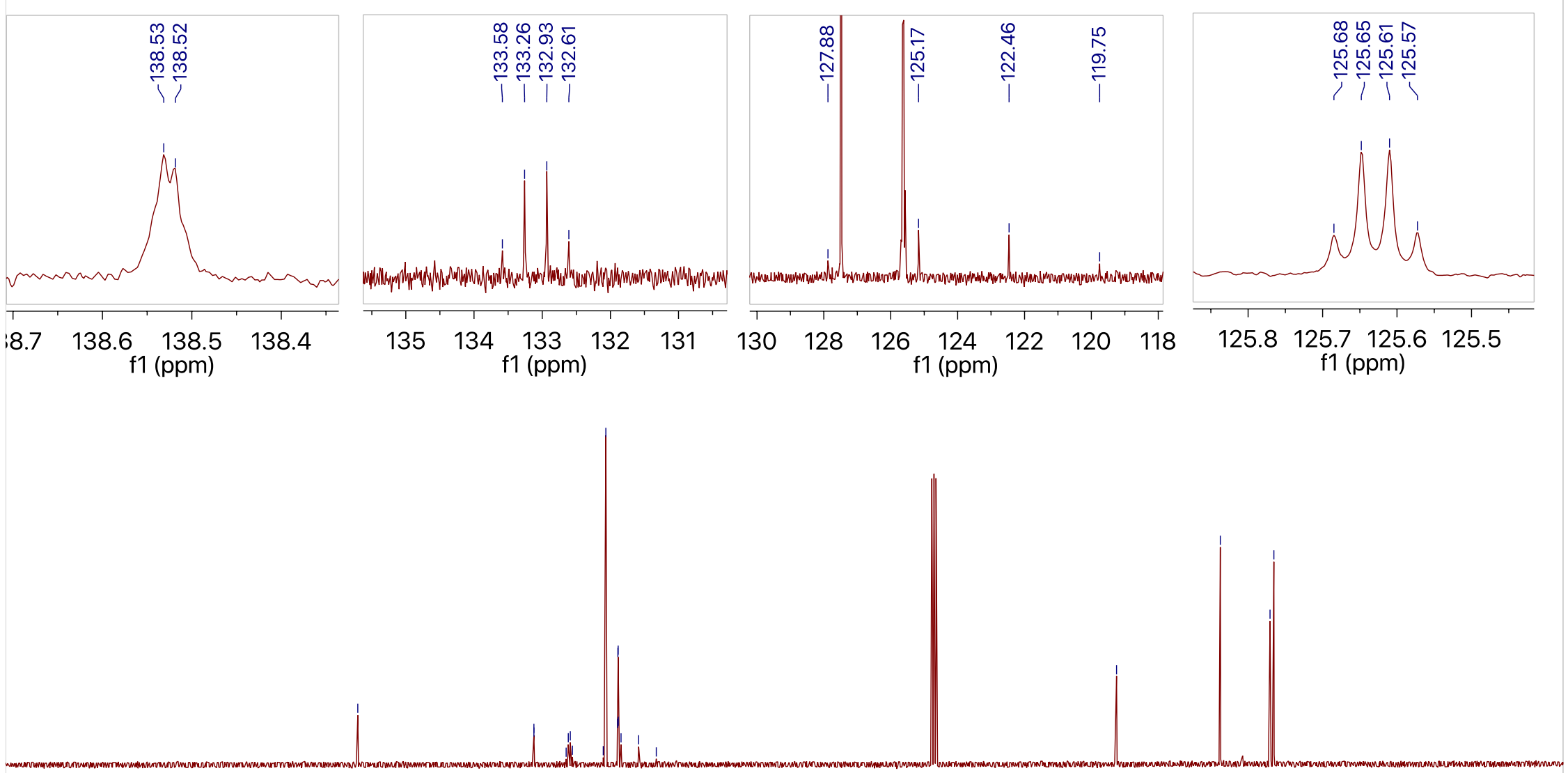

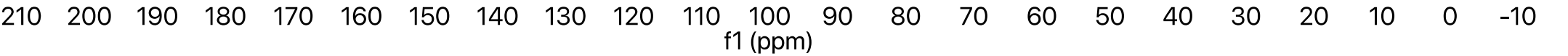


$\left.{ }^{19} \mathrm{~F} \mathrm{NMR} \mathrm{(376} \mathrm{MHz,} \mathrm{CDCl}_{3}\right)$

$$
\begin{aligned}
& \hat{o} \\
& 0 \\
& 0 \\
& 1
\end{aligned}
$$

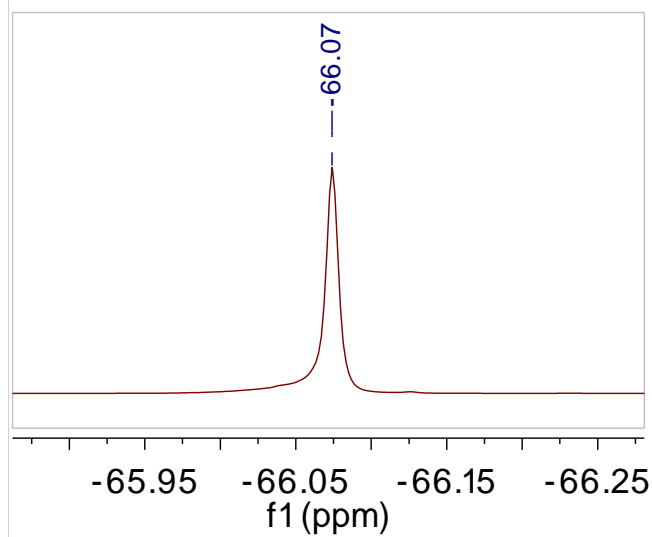

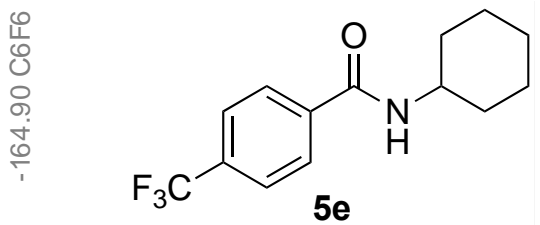

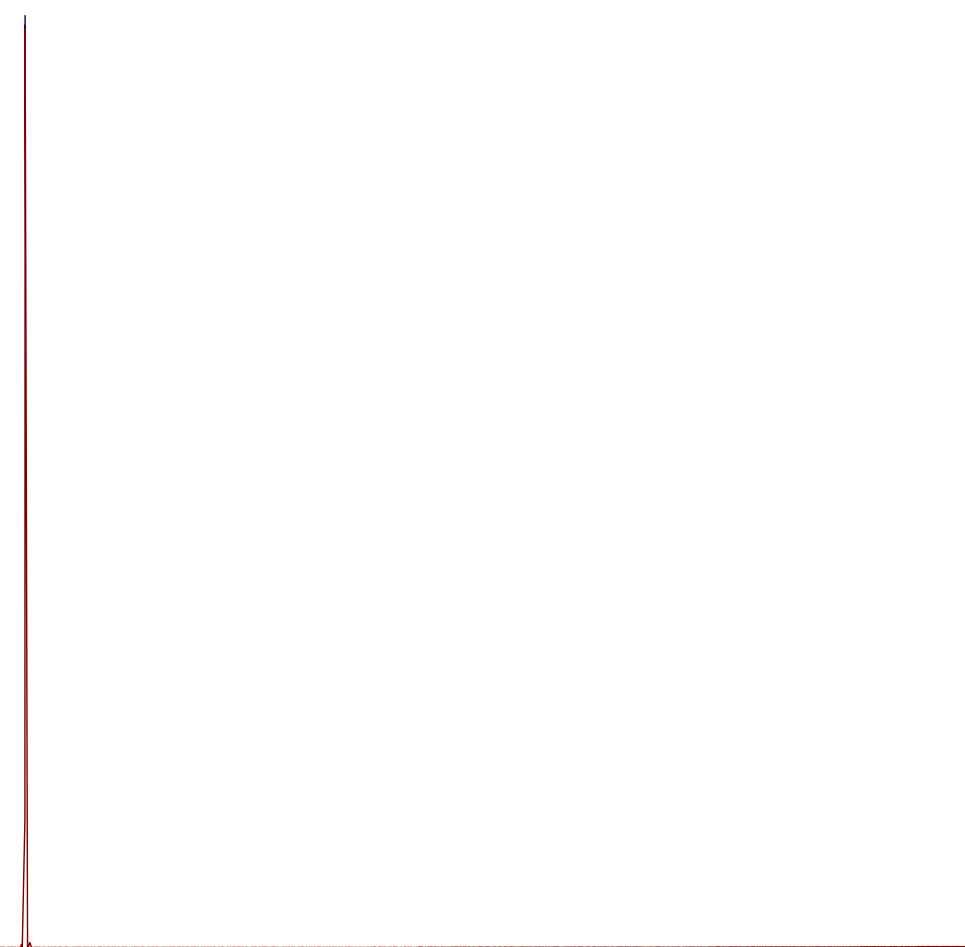

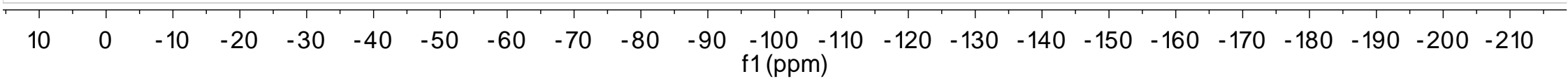




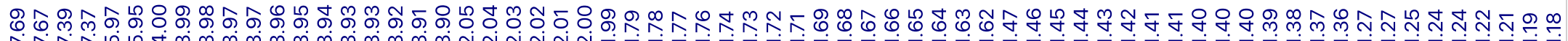

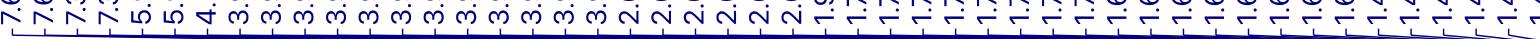
${ }^{1} \mathrm{H}$ NMR $\left(400 \mathrm{MHz}, \mathrm{CDCl}_{3}\right)$
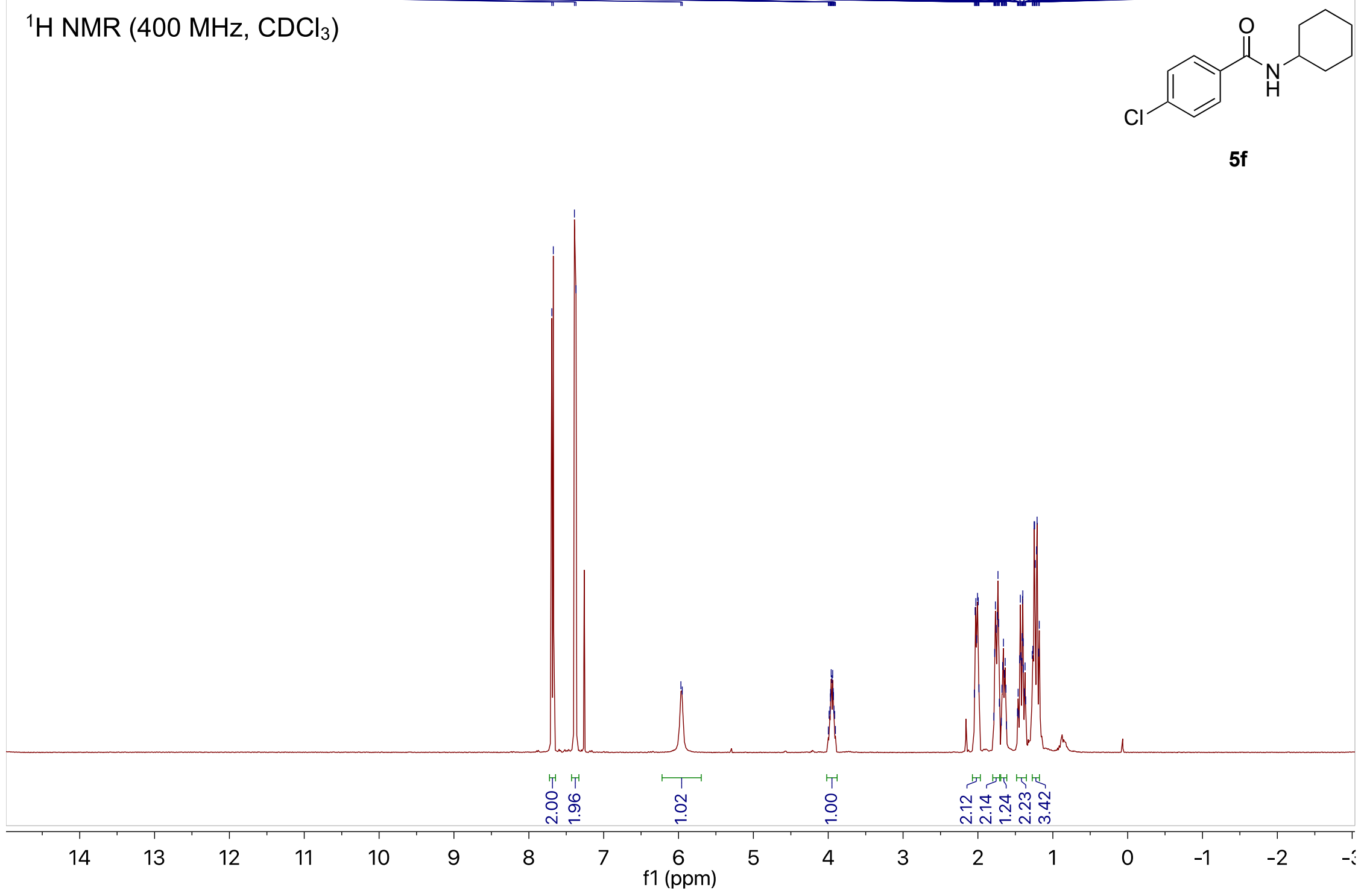


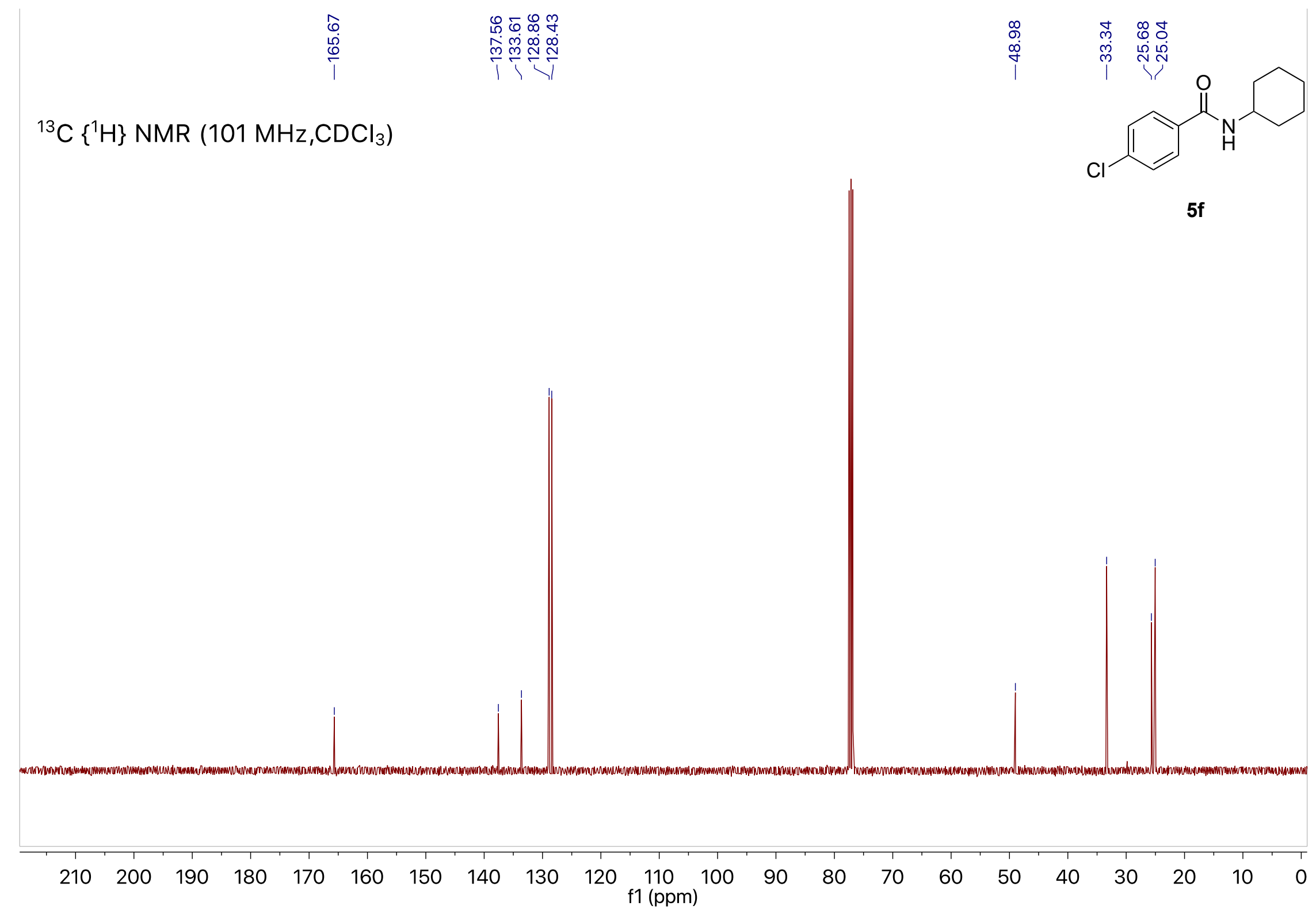




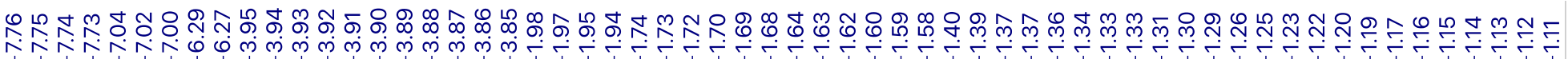
${ }^{1} \mathrm{H}$ NMR $\left(400 \mathrm{MHz}, \mathrm{CDCl}_{3}\right)$

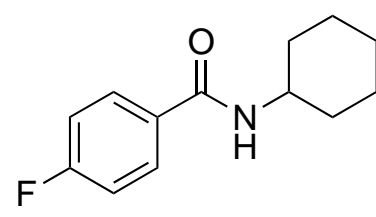

$5 g$

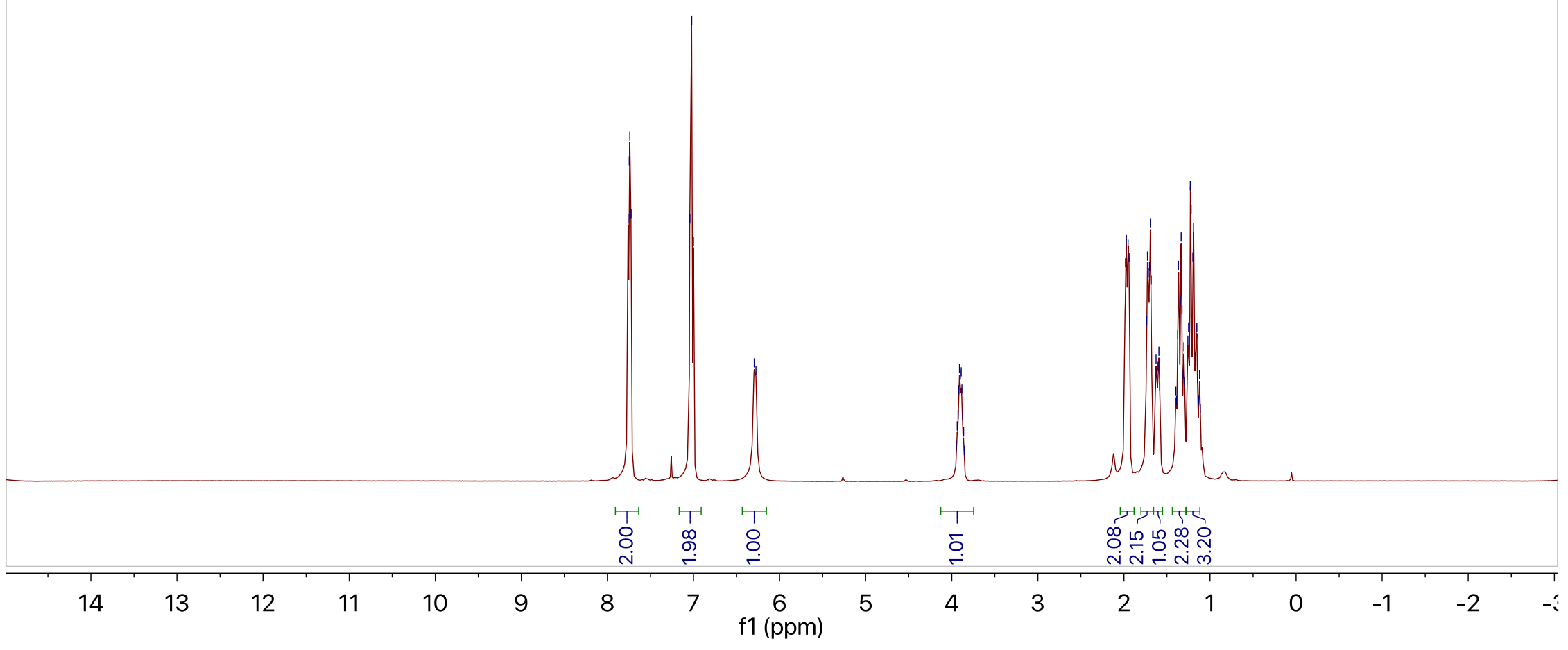




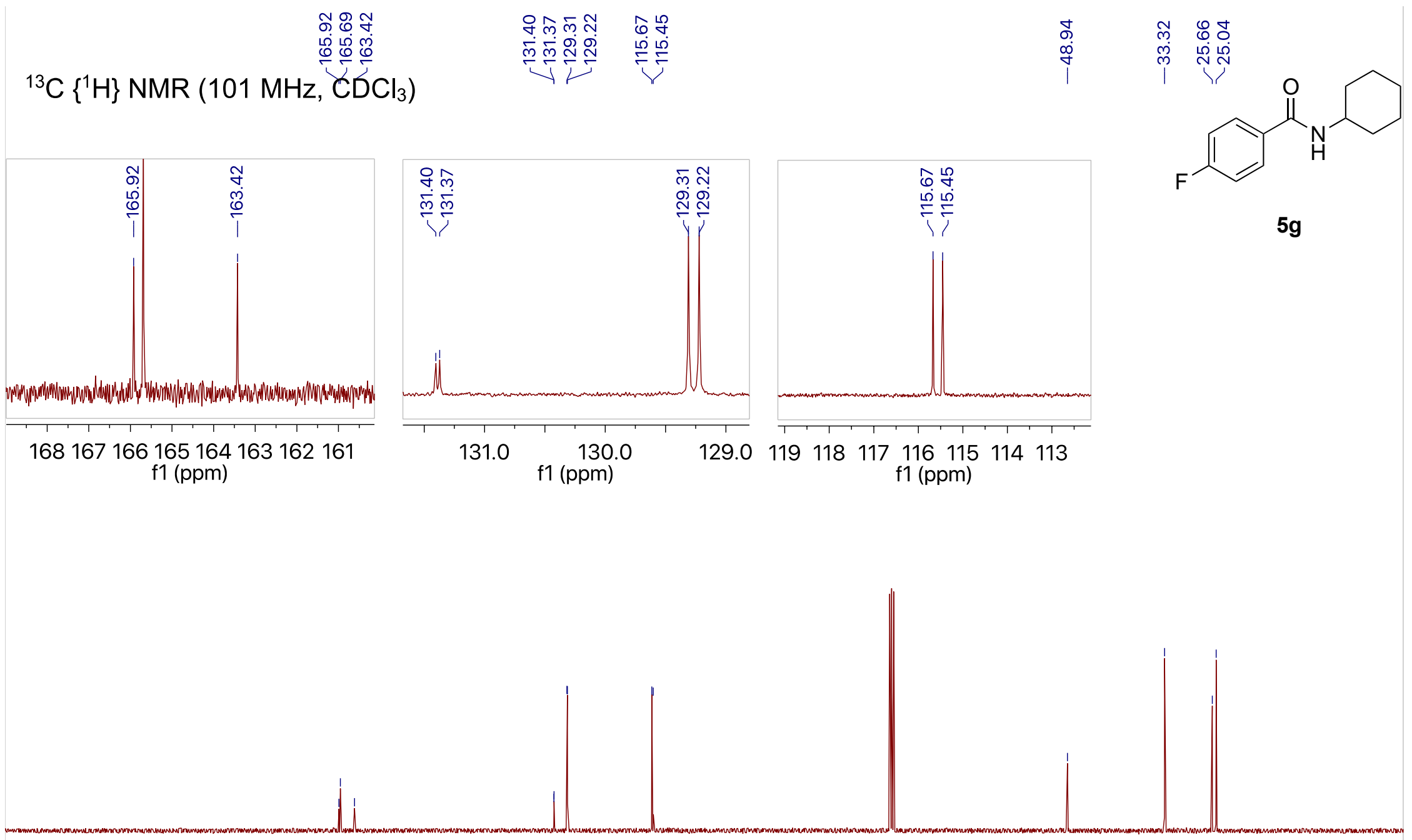

$\begin{array}{llllllllllllllllllllll}210 & 200 & 190 & 180 & 170 & 160 & 150 & 140 & 130 & 120 & \begin{array}{c}110 \\ f 1(\mathrm{ppm})\end{array} & 100 & 90 & 80 & 70 & 60 & 50 & 40 & 30 & 20 & 10 & 0\end{array}$ 
${ }^{19} \mathrm{~F} \mathrm{NMR} \mathrm{(376} \mathrm{MHz,} \mathrm{CDCl}_{3}$ )

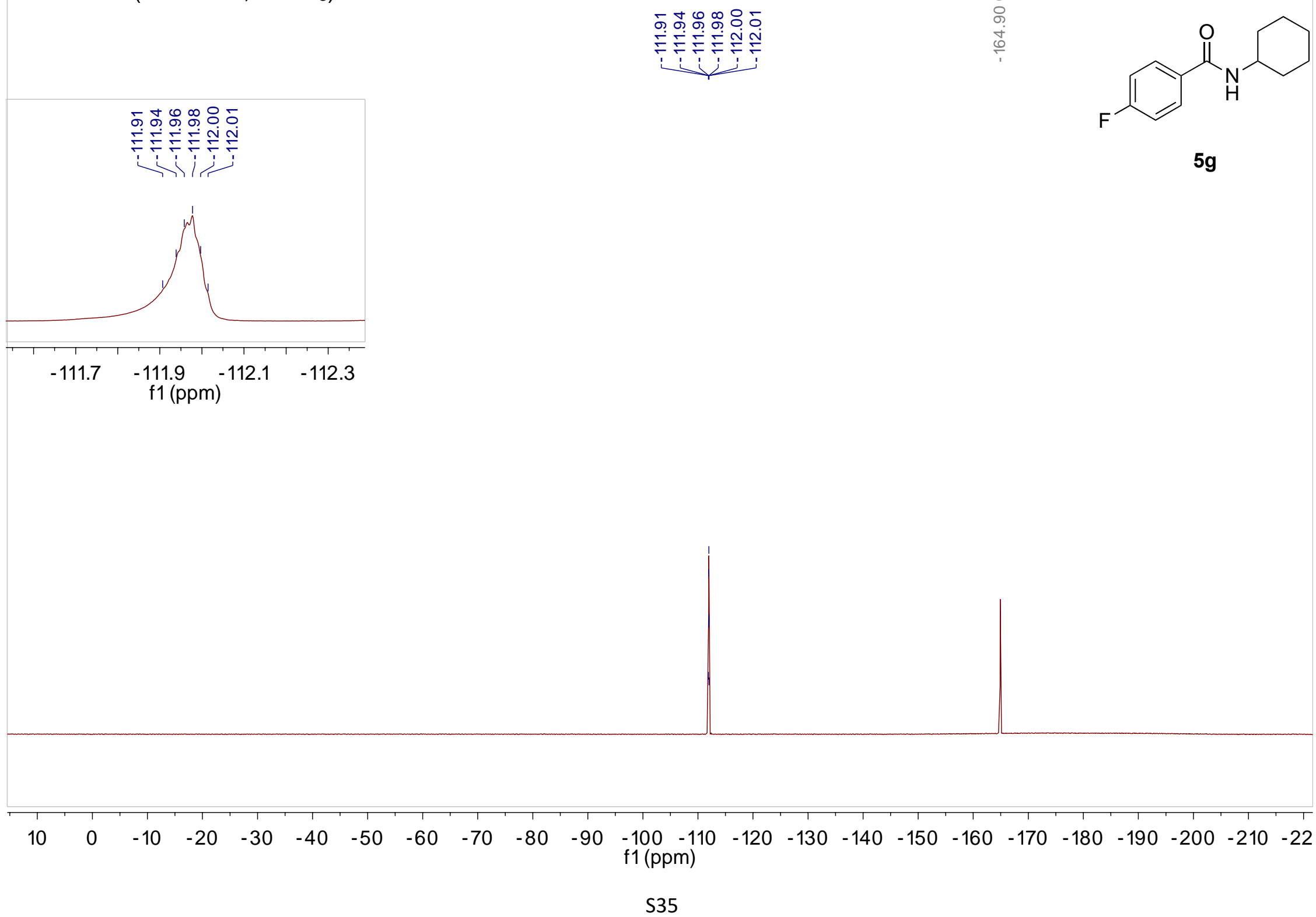




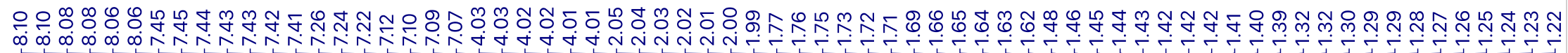
${ }^{1} \mathrm{H}$ NMR $\left(400 \mathrm{MHz}, \mathrm{CDCl}_{3}\right)$
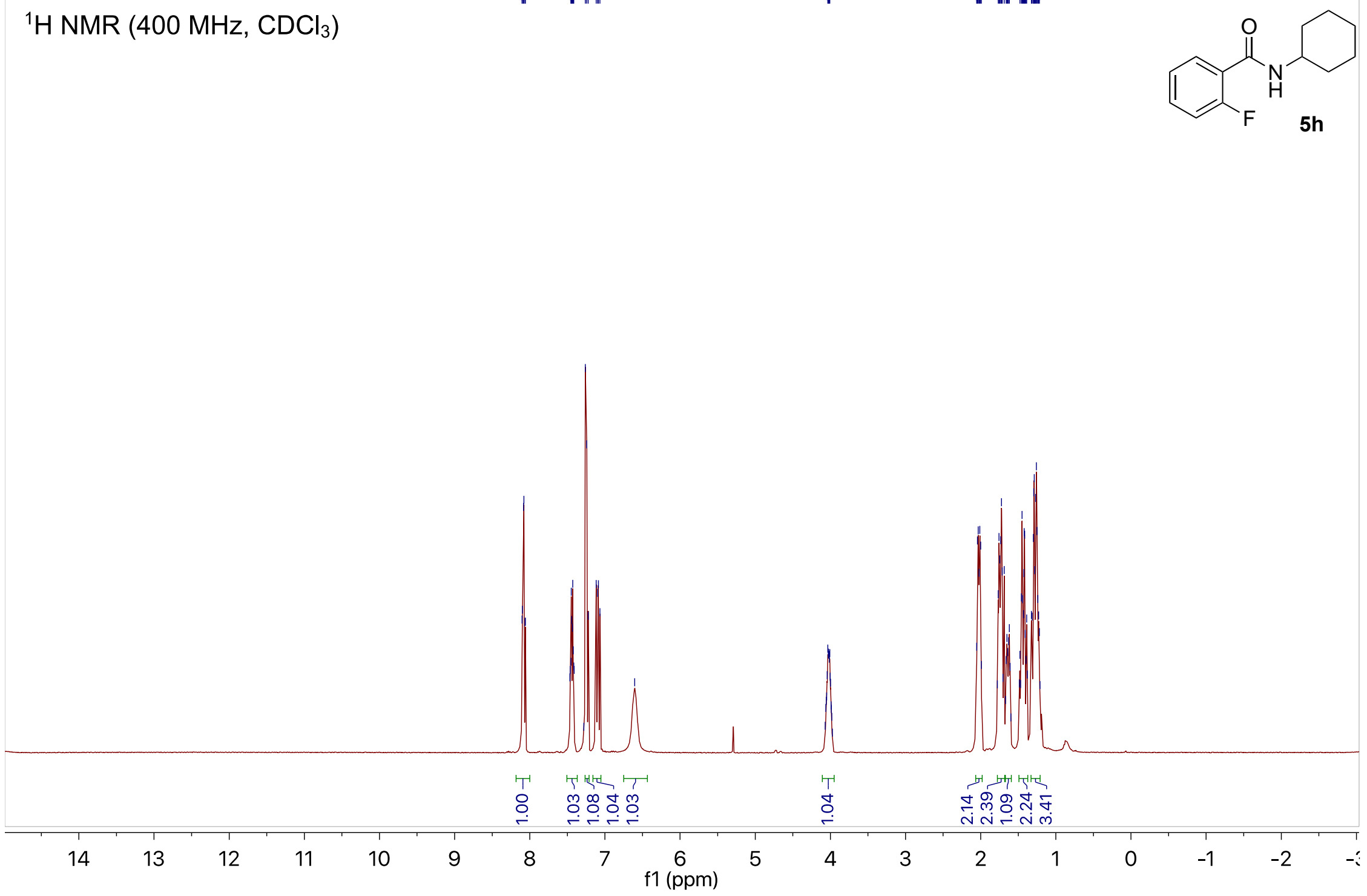

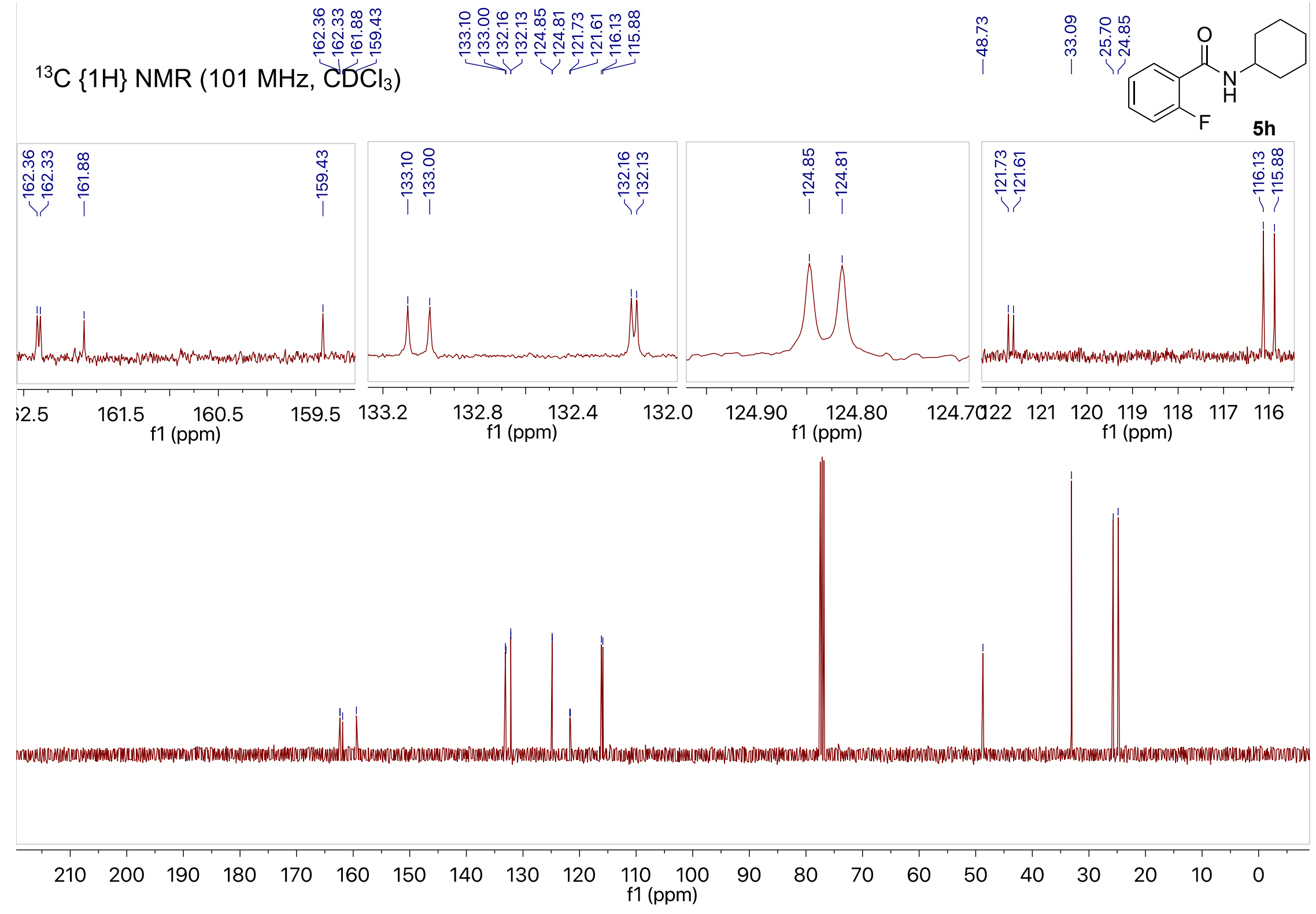
${ }^{19} \mathrm{~F} \mathrm{NMR}\left(376 \mathrm{MHz}, \mathrm{CDCl}_{3}\right)$

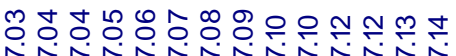

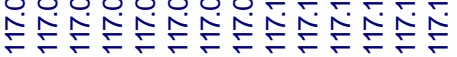

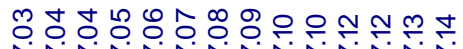

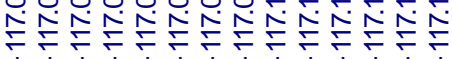

닌

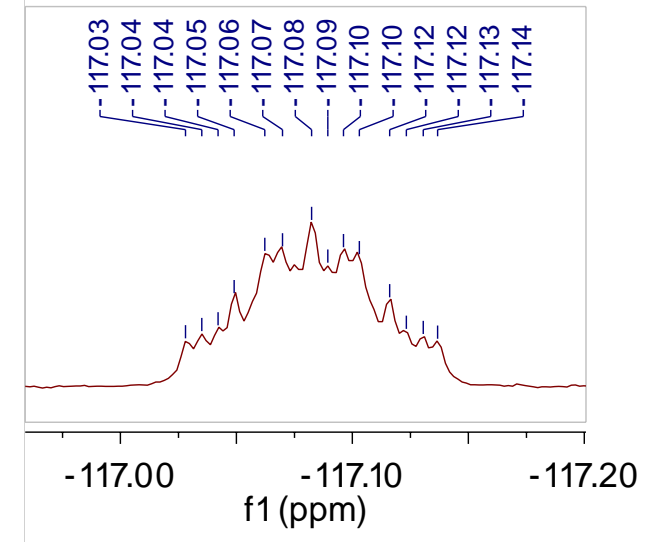

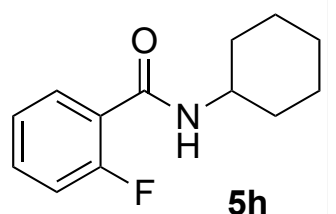

$5 \mathrm{~h}$

$\begin{array}{lllllllllllllllllllllllllll}10 & 0 & -10 & -20 & -30 & -40 & -50 & -60 & -70 & -80 & -90 & -100 & -110 & -120 & -130 & -140 & -150 & -160 & -170 & -180 & -190 & -200 & -210 & -22\end{array}$ 


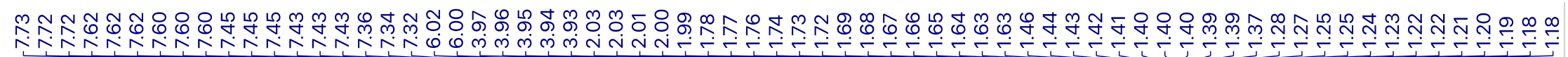
${ }^{1} \mathrm{H}$ NMR $\left(400 \mathrm{MHz}, \mathrm{CDCl}_{3}\right)$

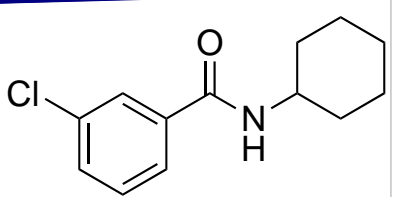

$5 i$

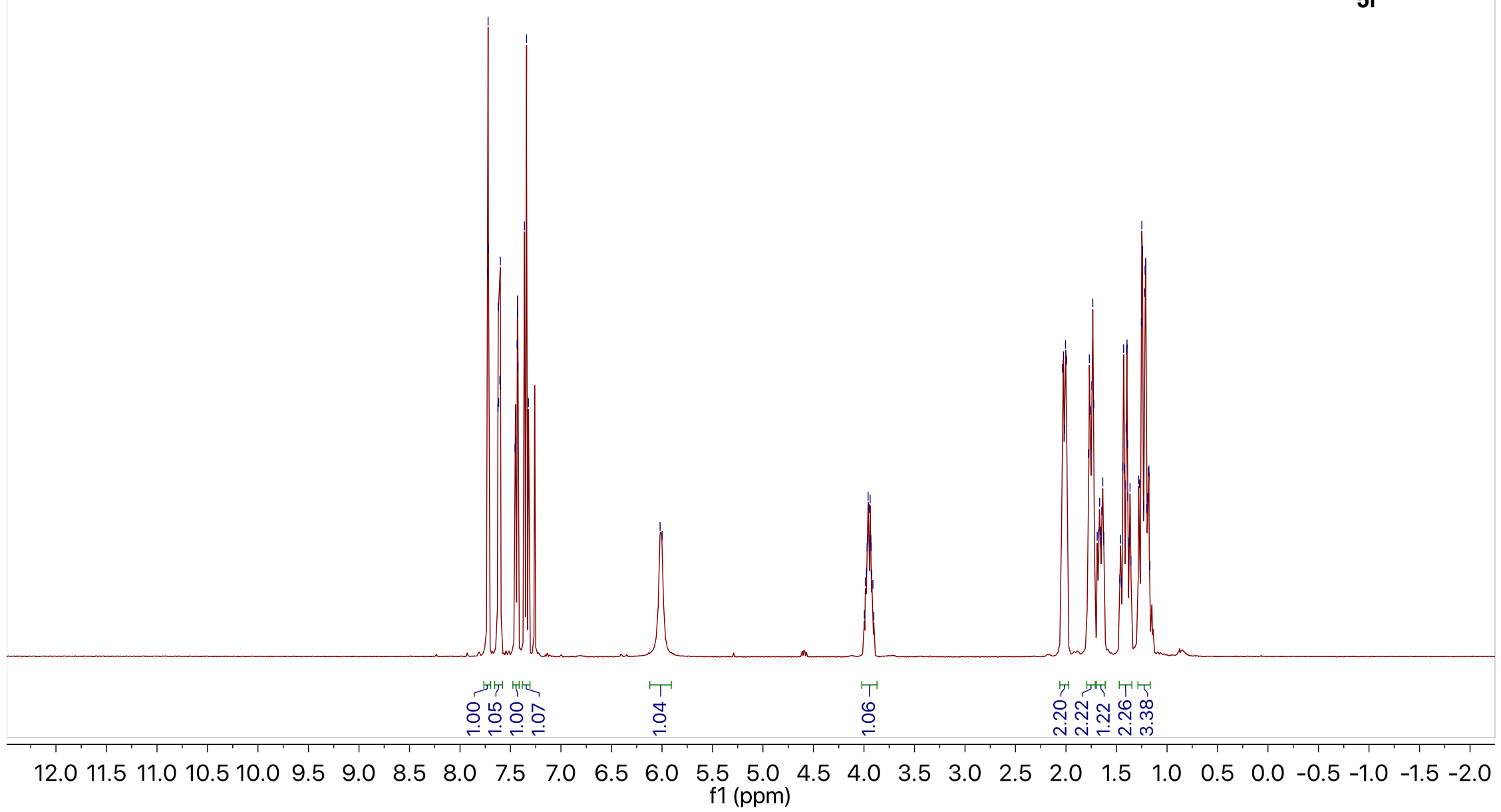




$$
{ }^{13} \mathrm{C}\{1 \mathrm{H}\} \mathrm{NMR}(101 \mathrm{MHz}, \mathrm{CDCl})
$$$$
\text { ग) }
$$

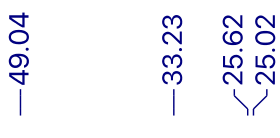<smiles>O=C(NC1CCCCC1)c1cccc(Cl)c1</smiles>

$5 i$

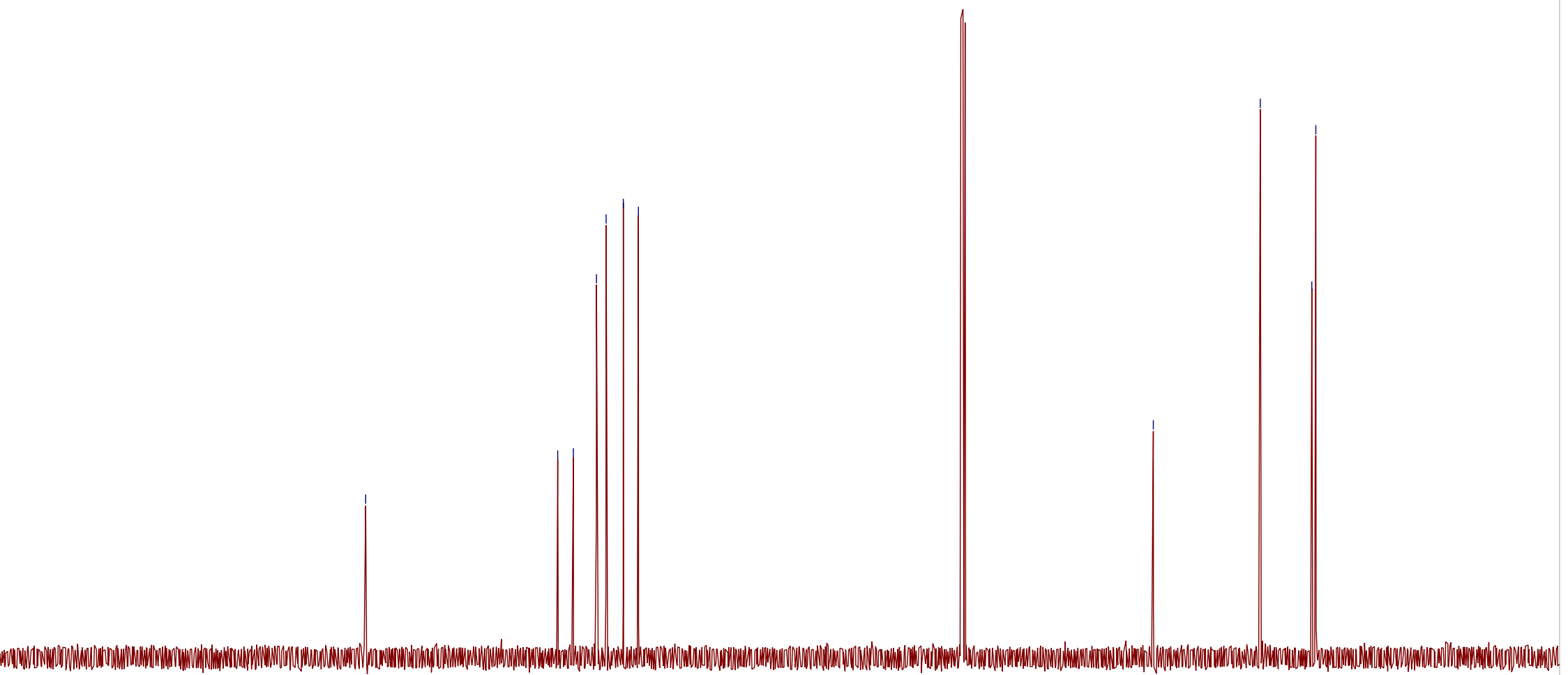

$210 \quad 200$

190180

$170 \quad 160$

150

130

$120 \quad 110$ f1 (ppm) 


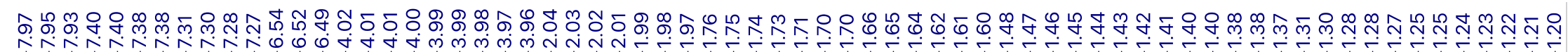
${ }^{1} \mathrm{H}$ NMR (400 $\left.\mathrm{MHz}, \mathrm{CDCl}_{3}\right)$
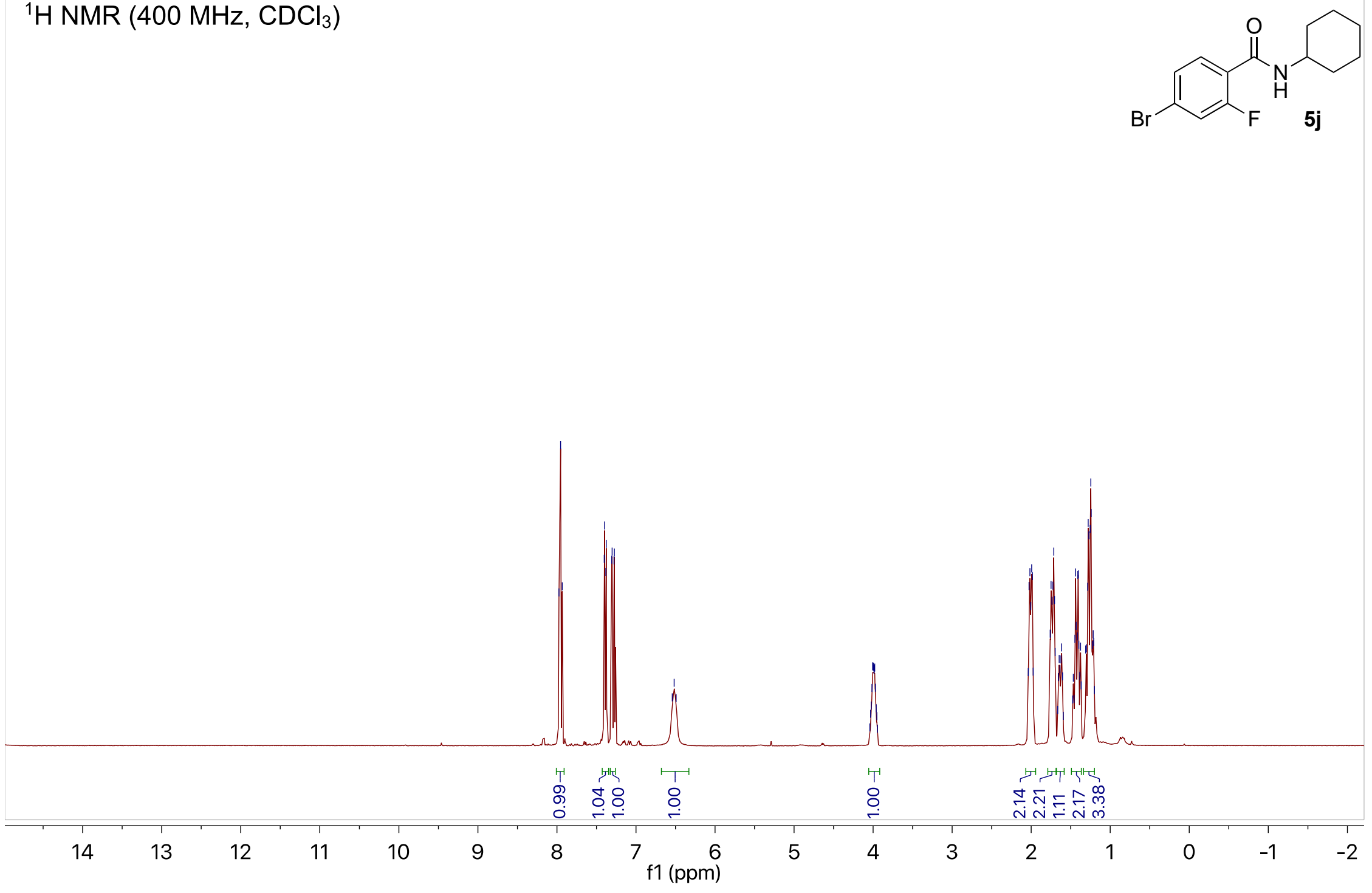


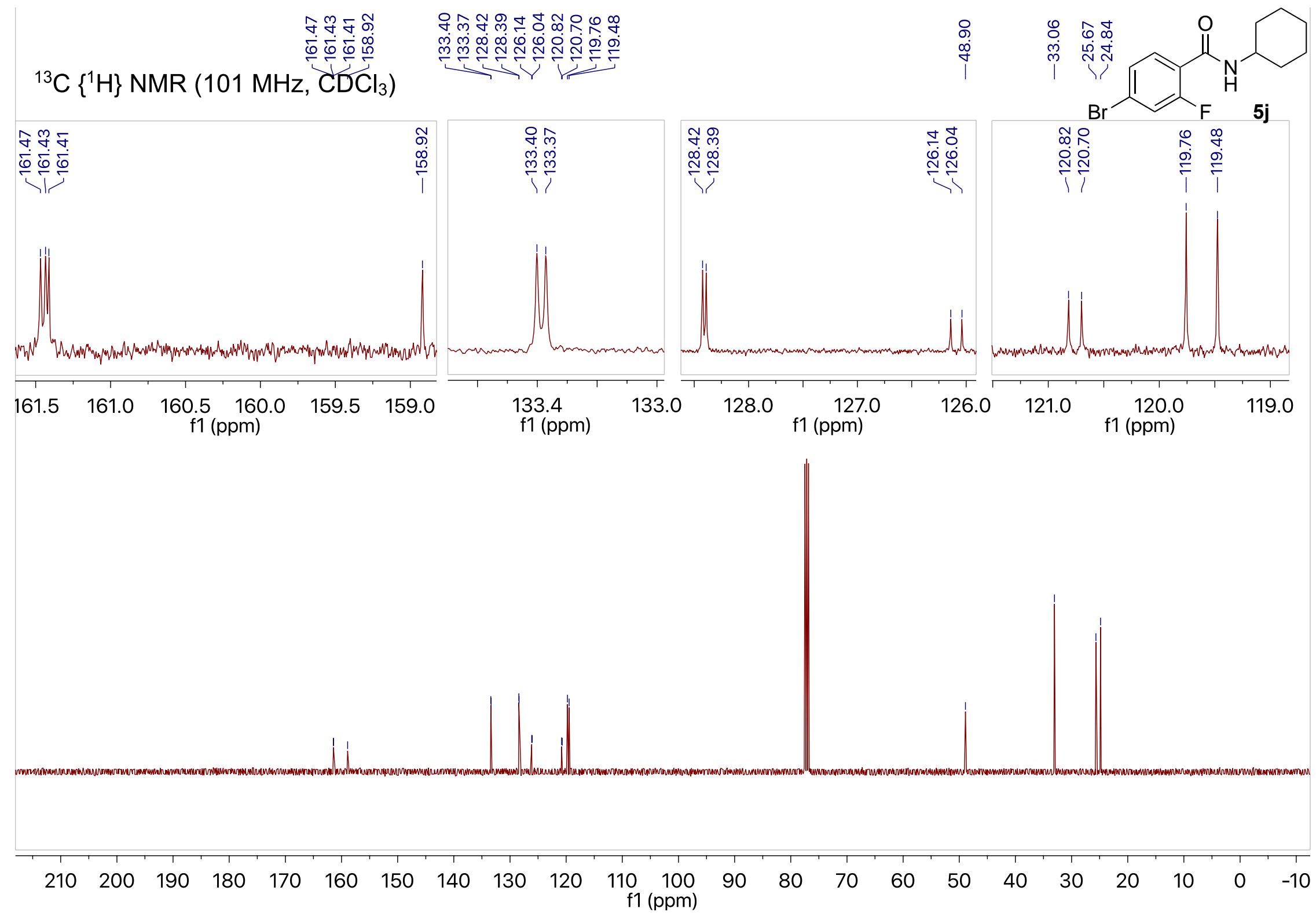


${ }^{19} \mathrm{~F}$ NMR $\left(376 \mathrm{MHz}, \mathrm{CDCl}_{3}\right.$ )

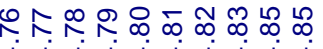

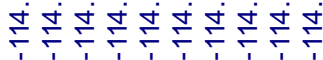

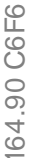

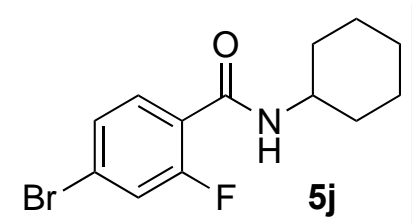

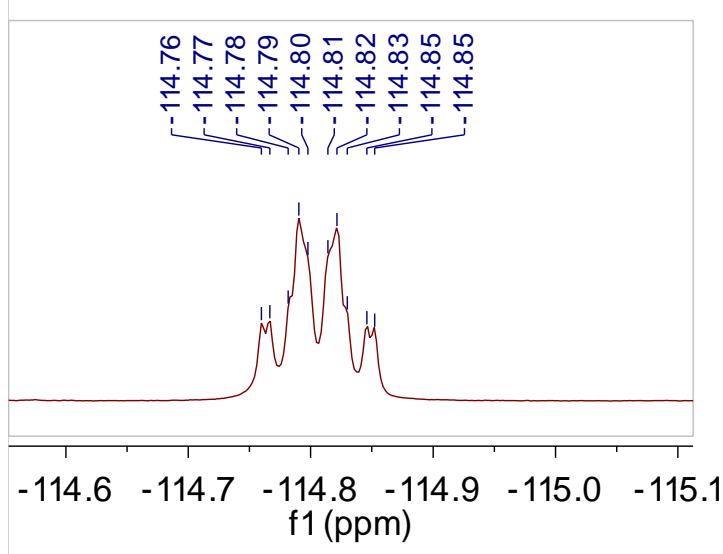

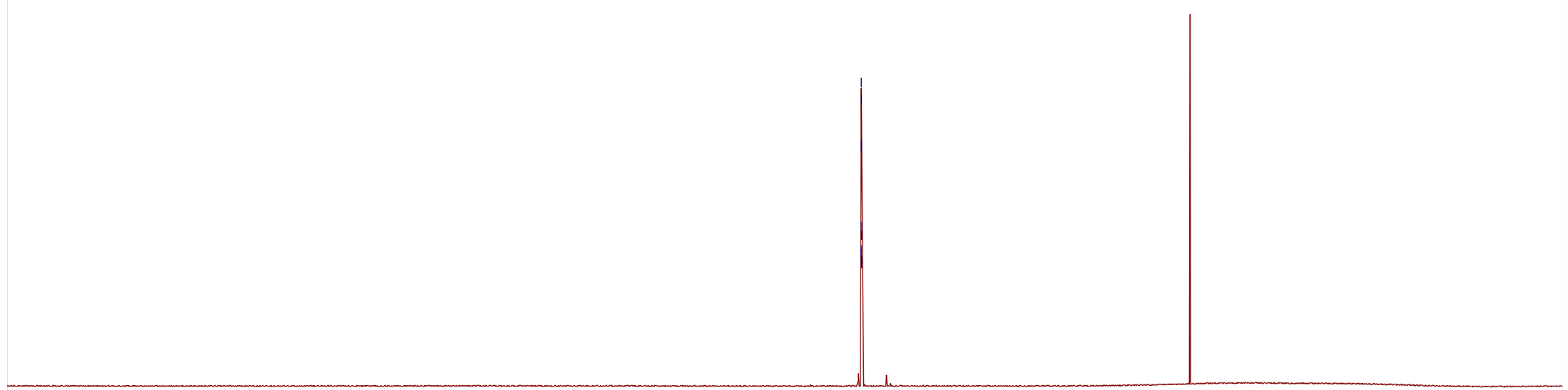

\begin{tabular}{|c|c|c|c|c|c|c|c|c|c|c|c|c|c|c|c|c|c|c|}
\hline 10 & 0 & -10 & -20 & -30 & -40 & -50 & -60 & - 70 & -80 & -90 & $\begin{array}{l}-100-110 \\
\text { f1 (ppm) }\end{array}$ & -120 & -130 & -140 & -150 & -160 & -170 & $\begin{array}{llll}-180 & -190 & -200 & -210\end{array}$ \\
\hline
\end{tabular}




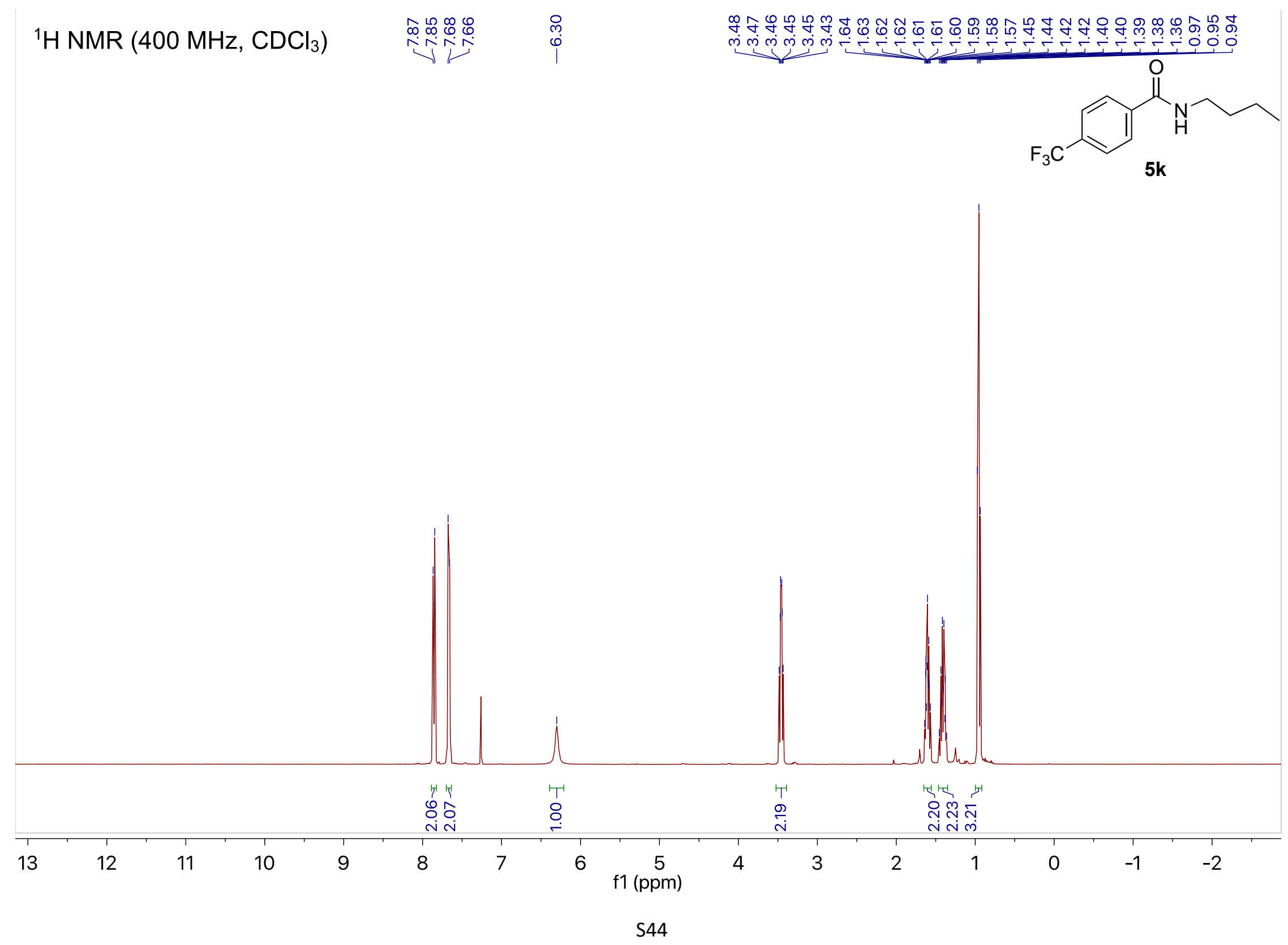



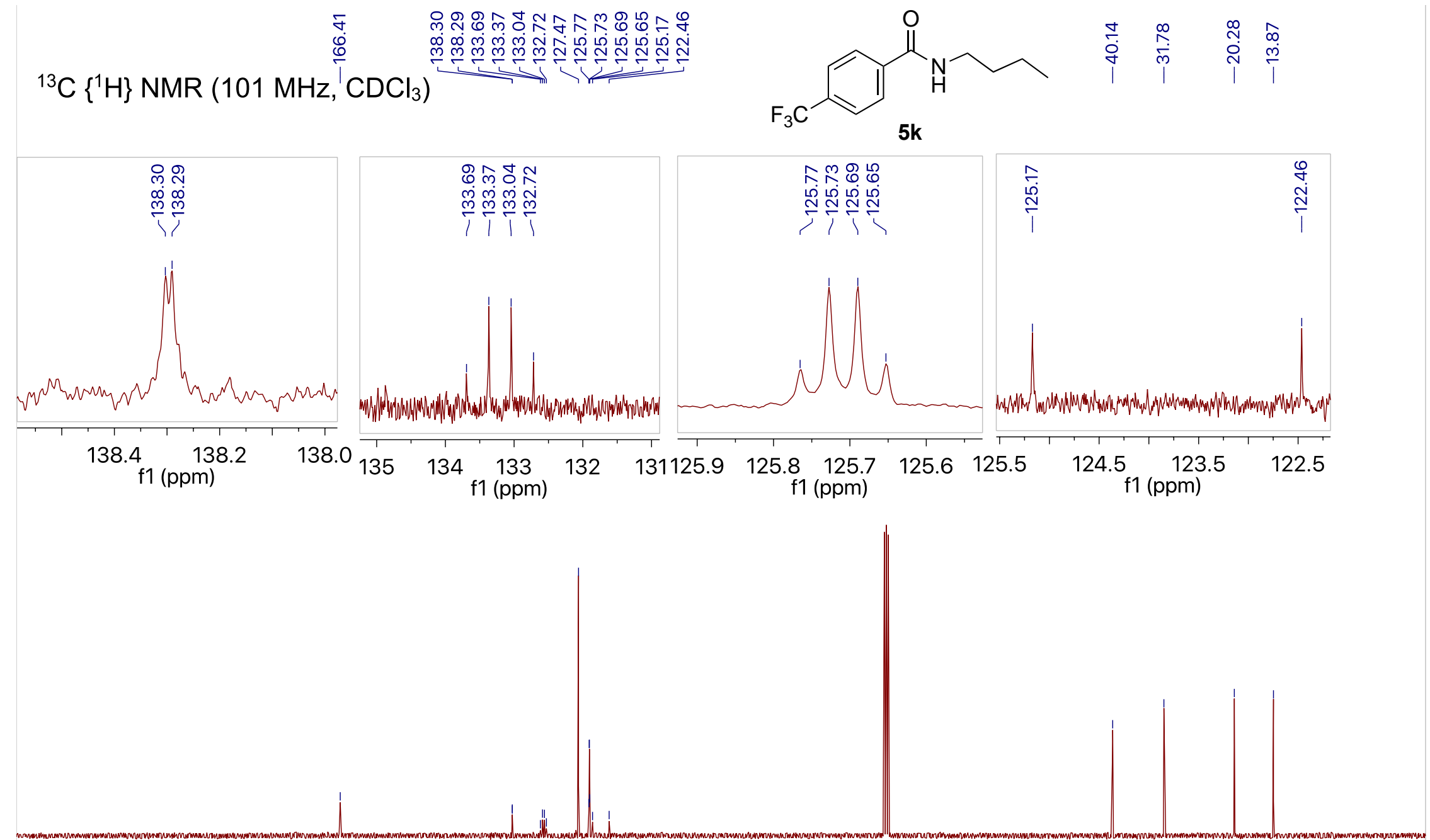

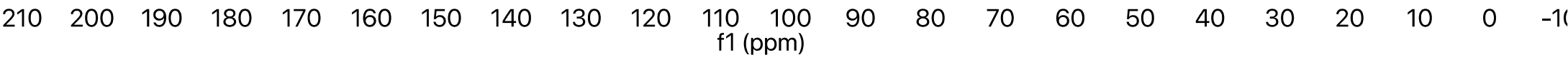


${ }^{19} \mathrm{~F} \mathrm{NMR}\left(376 \mathrm{MHz}, \mathrm{CDCl}_{3}\right)$

$$
\begin{aligned}
& \text { 우울 } \\
& 0.0 \\
& 0 \\
& \text { Y }
\end{aligned}
$$

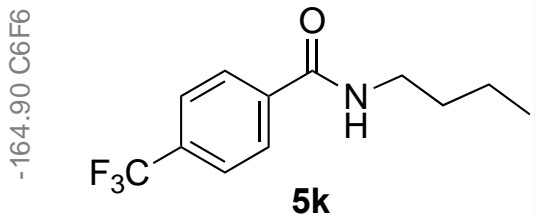

5
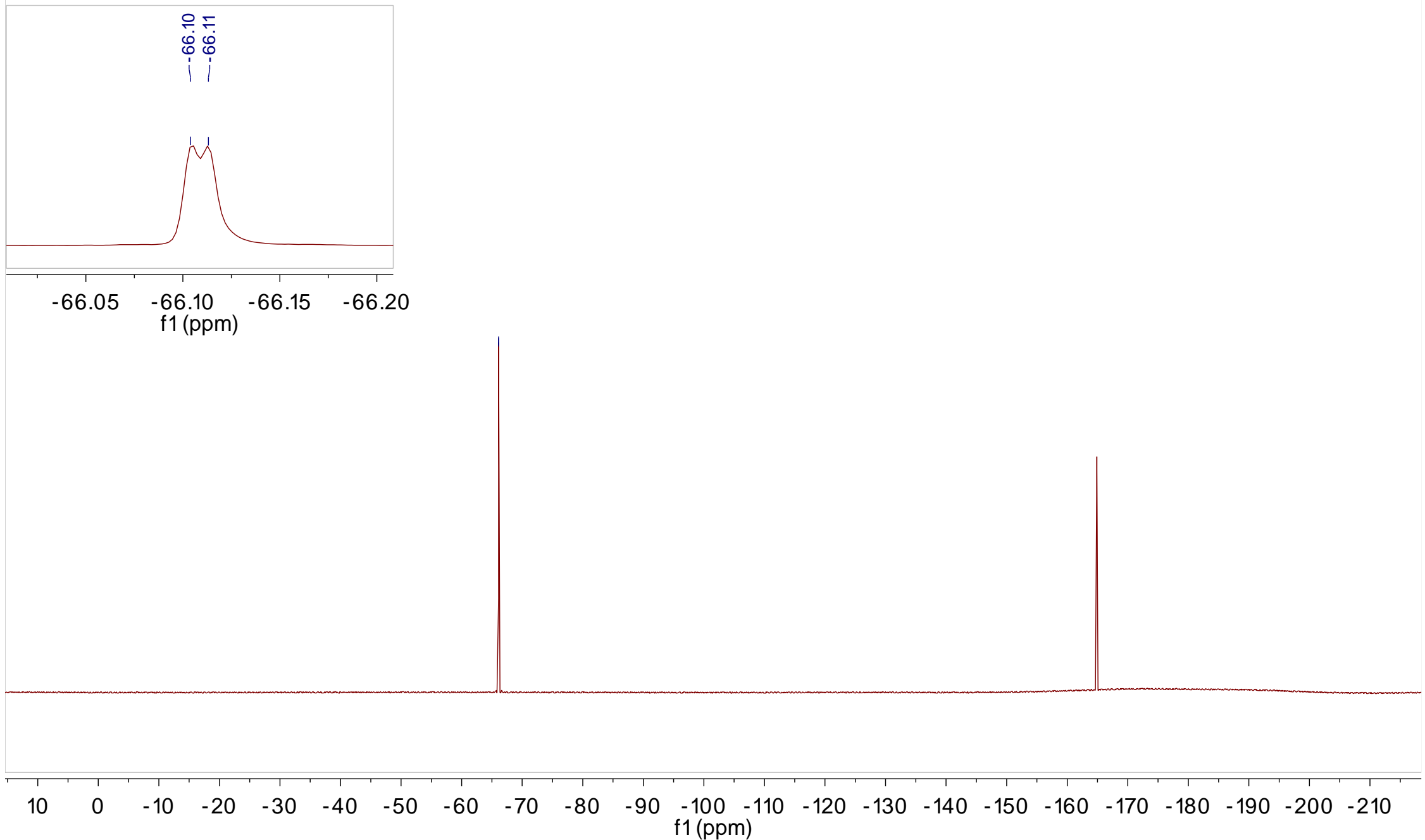


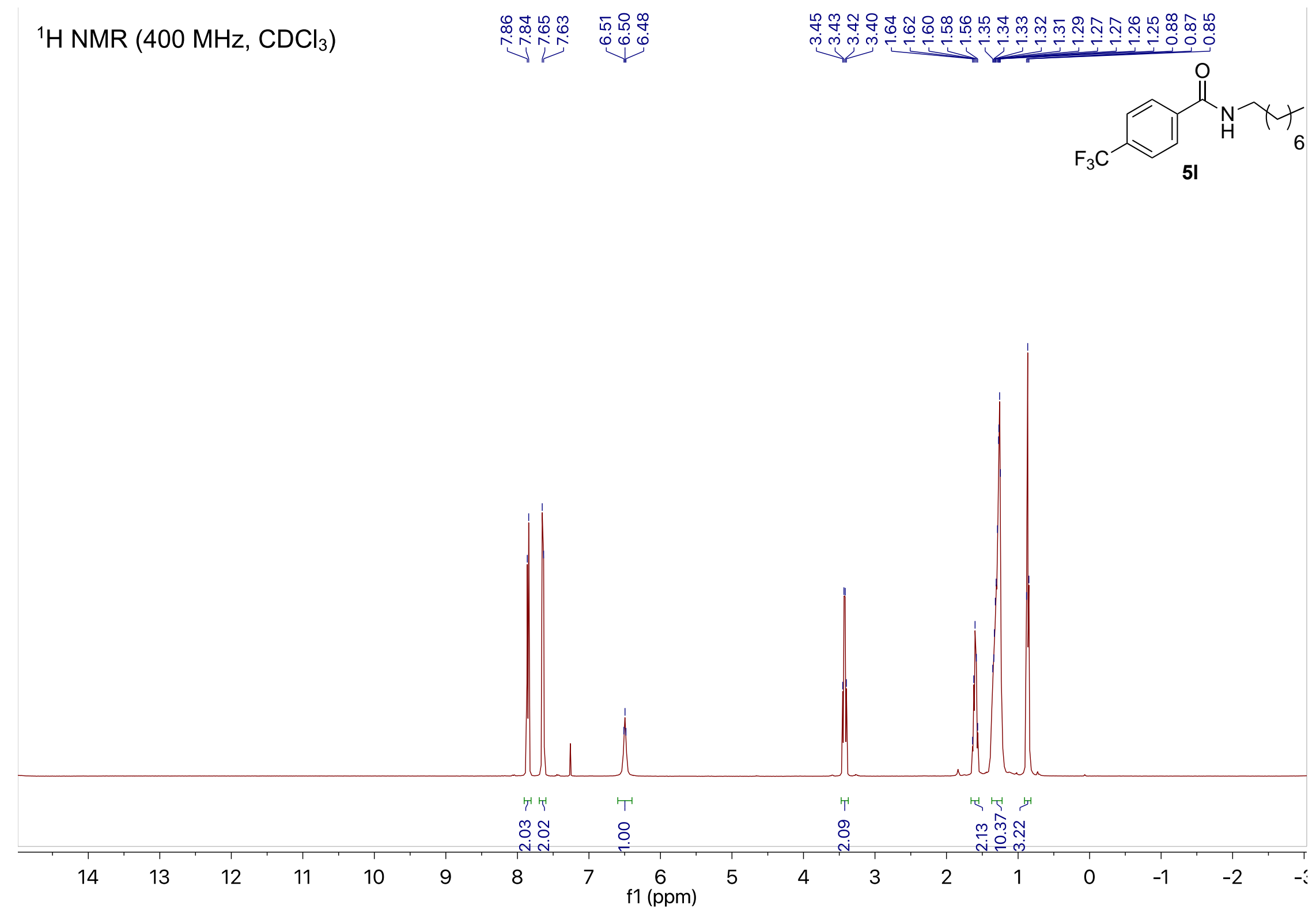



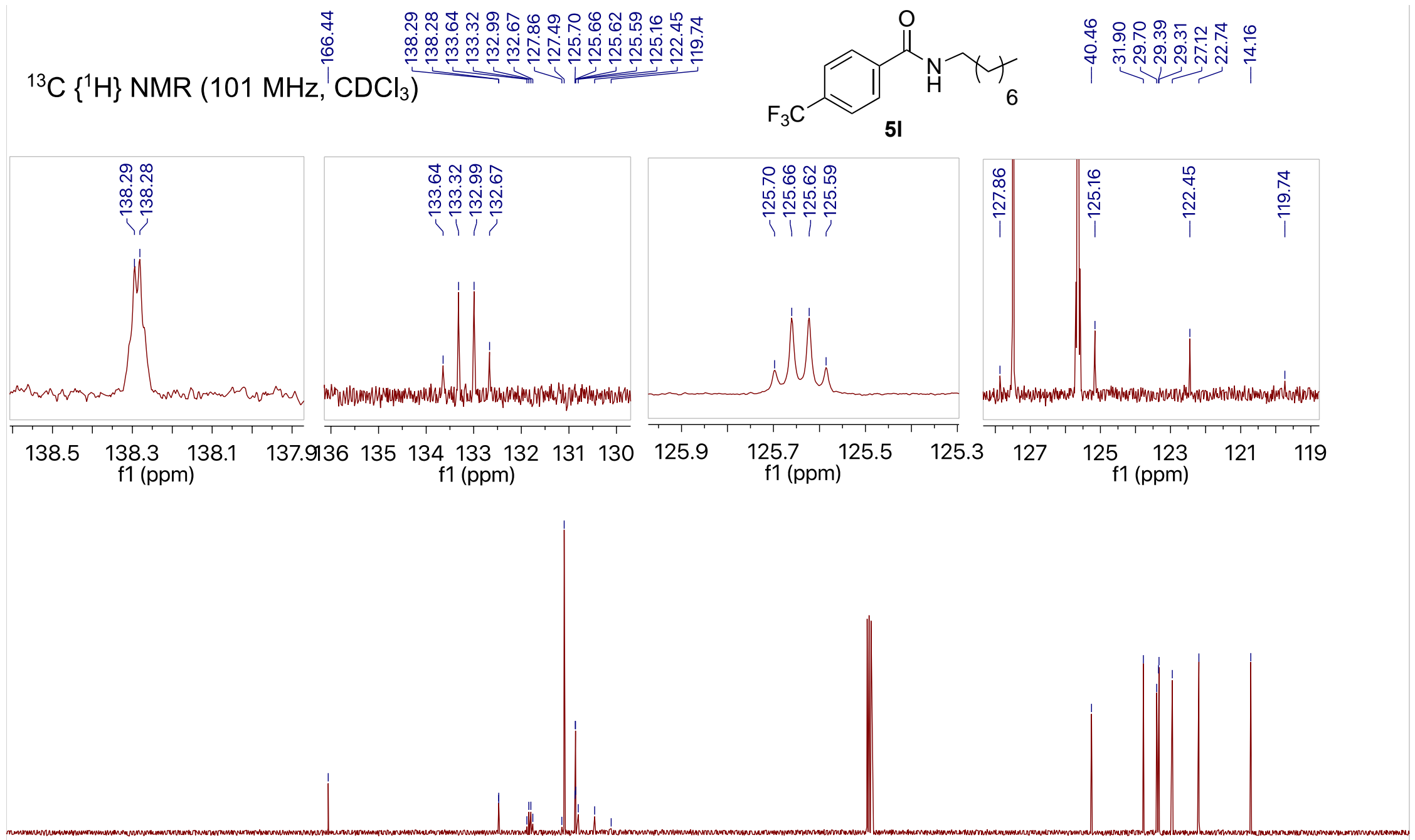

$\begin{array}{lllllllllllllllllllllll}210 & 200 & 190 & 180 & 170 & 160 & 150 & 140 & 130 & 120 & \begin{array}{r}110 \\ \mathrm{f} 1(\mathrm{ppm})\end{array} & 90 & 80 & 70 & 60 & 50 & 40 & 30 & 20 & 10 & 0 & -10\end{array}$ 
$\left.{ }^{19} \mathrm{~F} \mathrm{NMR} \mathrm{(376} \mathrm{MHz,} \mathrm{CDCl}_{3}\right)$

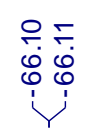

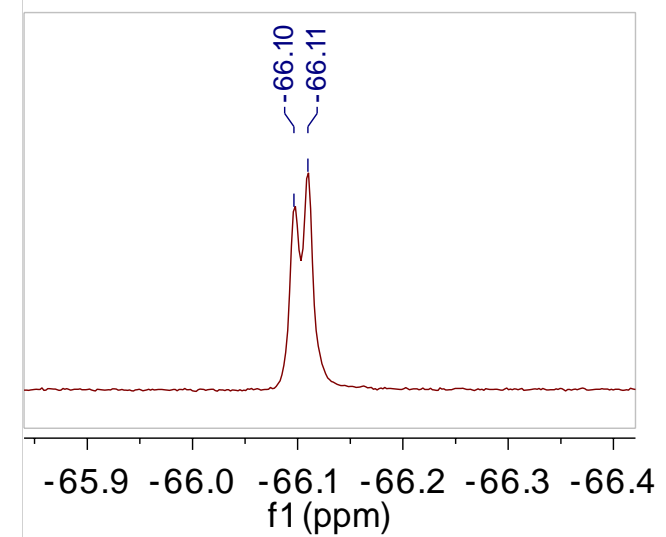

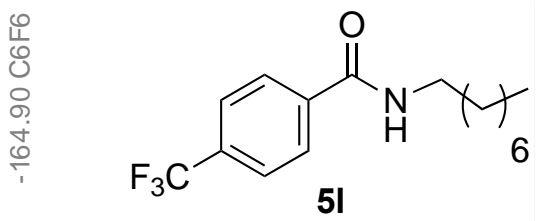

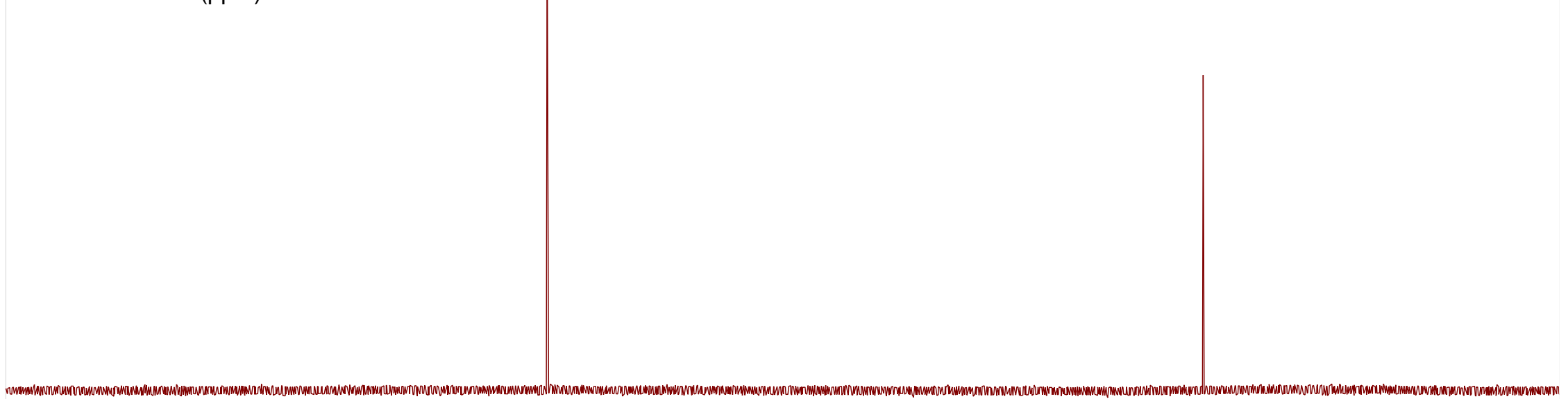

$\begin{array}{llllllllllllllllllllllllll}10 & 0 & -10 & -20 & -30 & -40 & -50 & -60 & -70 & -80 & -90 & -100 & -110 & -120 & -130 & -140 & -150 & -160 & -170 & -180 & -190 & -200 & -210\end{array}$



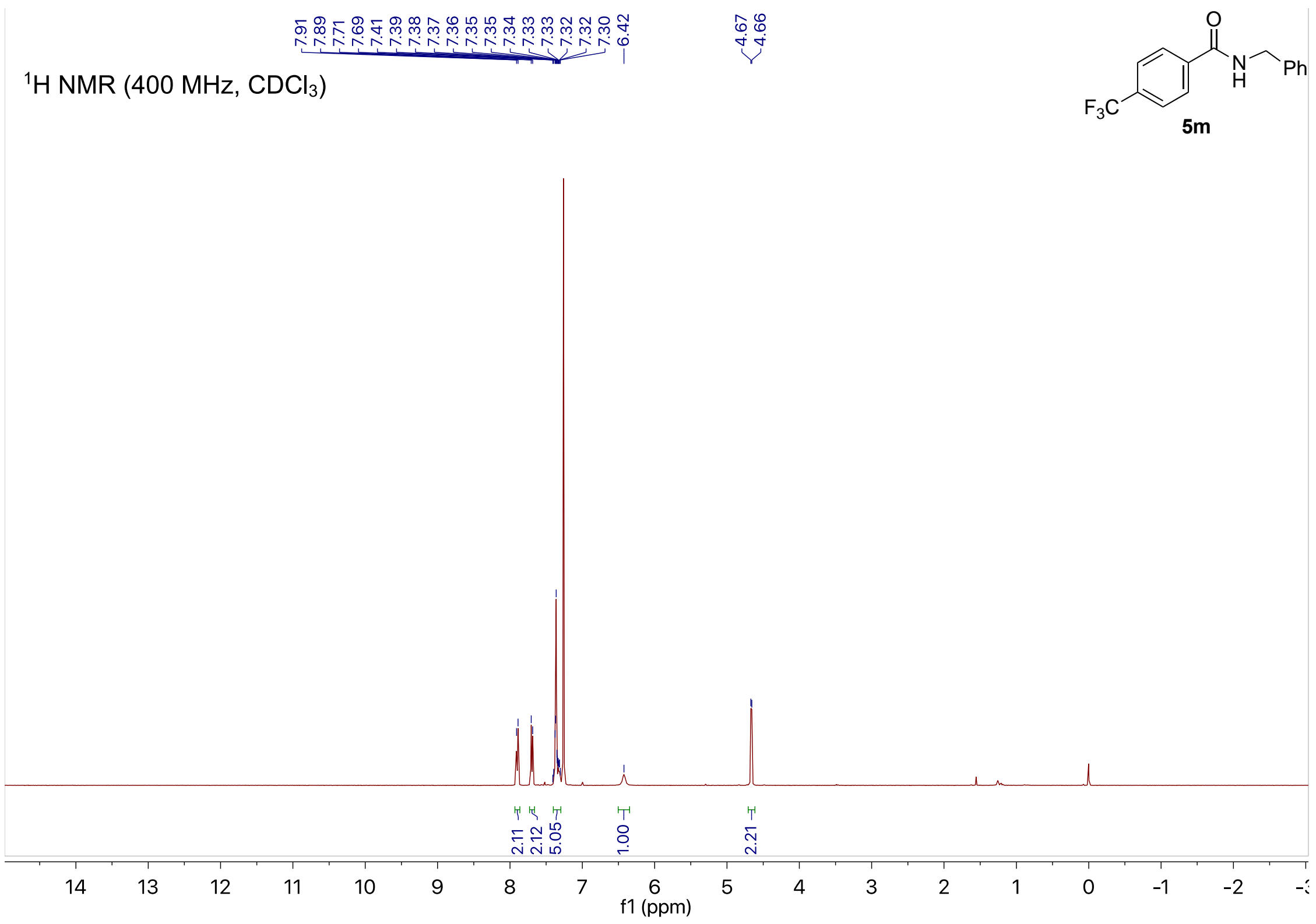
${ }^{13} \mathrm{C}\left\{{ }^{1} \mathrm{H}\right\}$ NMR $\left(101 \mathrm{MHz}, \mathrm{CDCl}_{3}\right)$
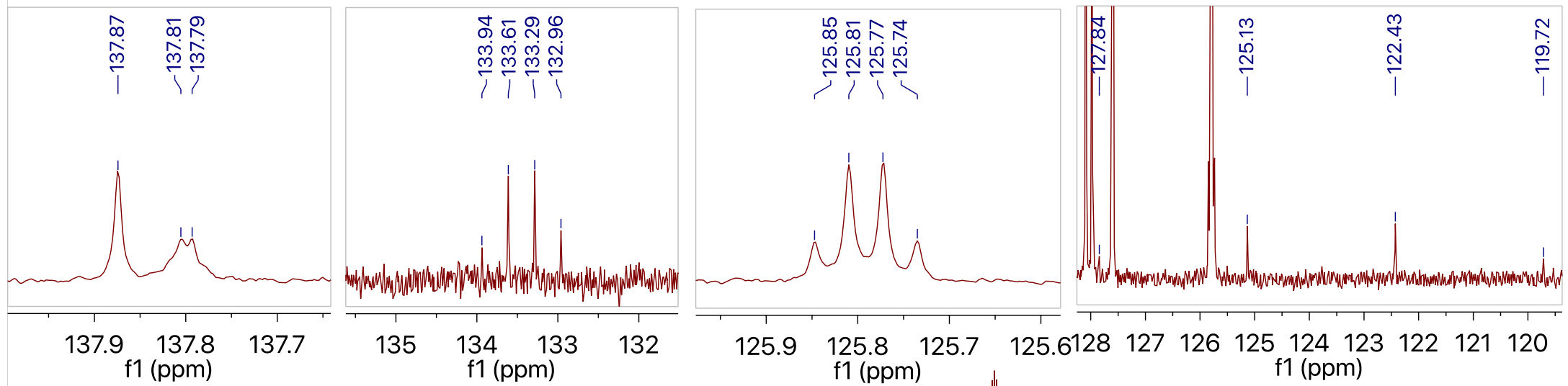

f1 (ppm)

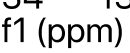

f1 (ppm)

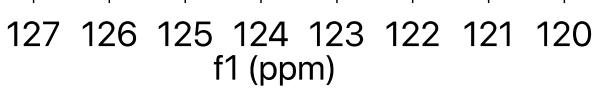

$\stackrel{+10}{+\infty}$

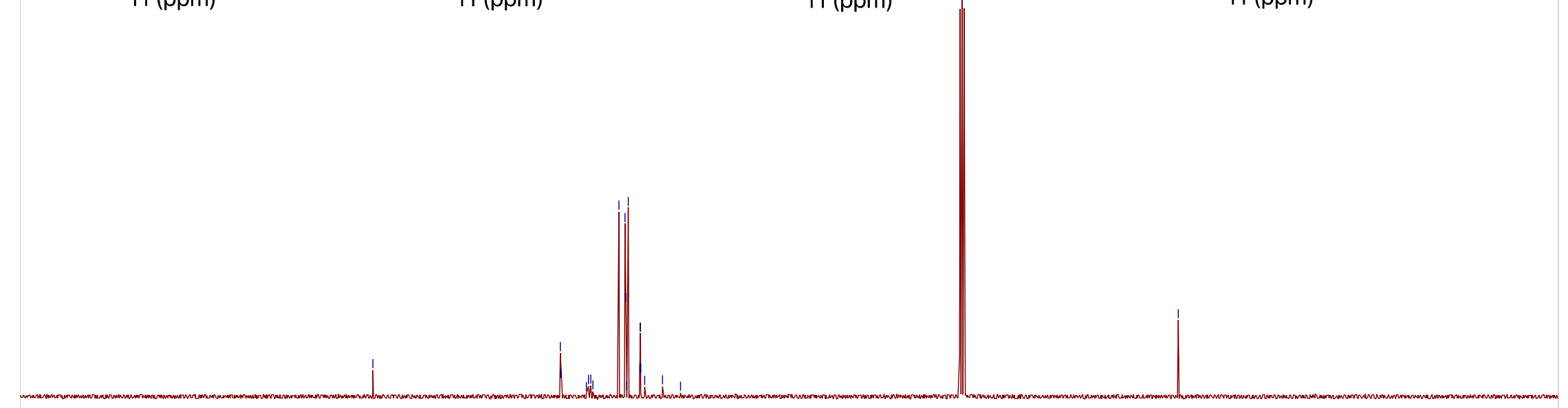

$\begin{array}{rrrrrrrrrrrrrrrrrrrrrrr}210 & 200 & 190 & 180 & 170 & 160 & 150 & 140 & 130 & 120 & \begin{array}{r}110 \\ \mathrm{f} 1(\mathrm{ppm})\end{array} & 90 & 80 & 70 & 60 & 50 & 40 & 30 & 20 & 10 & 0 & -10\end{array}$ 
${ }^{19} \mathrm{~F} \mathrm{NMR}\left(376 \mathrm{MHz}, \mathrm{CDCl}_{3}\right)$

$\stackrel{\substack{0 \\ \dot{0}}}{\underline{i}}$

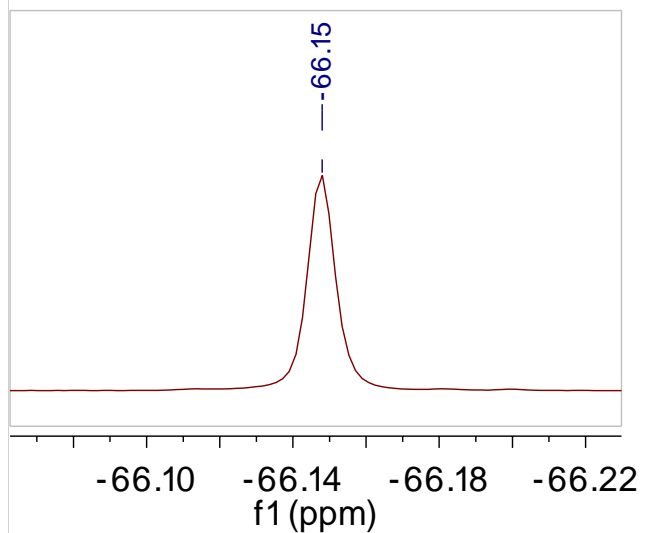

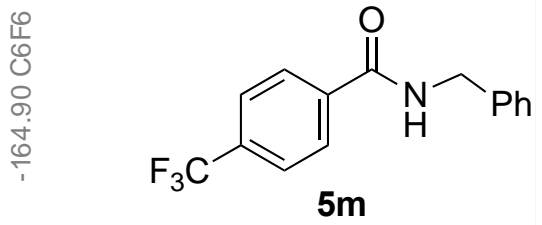

$5 \mathrm{~m}$

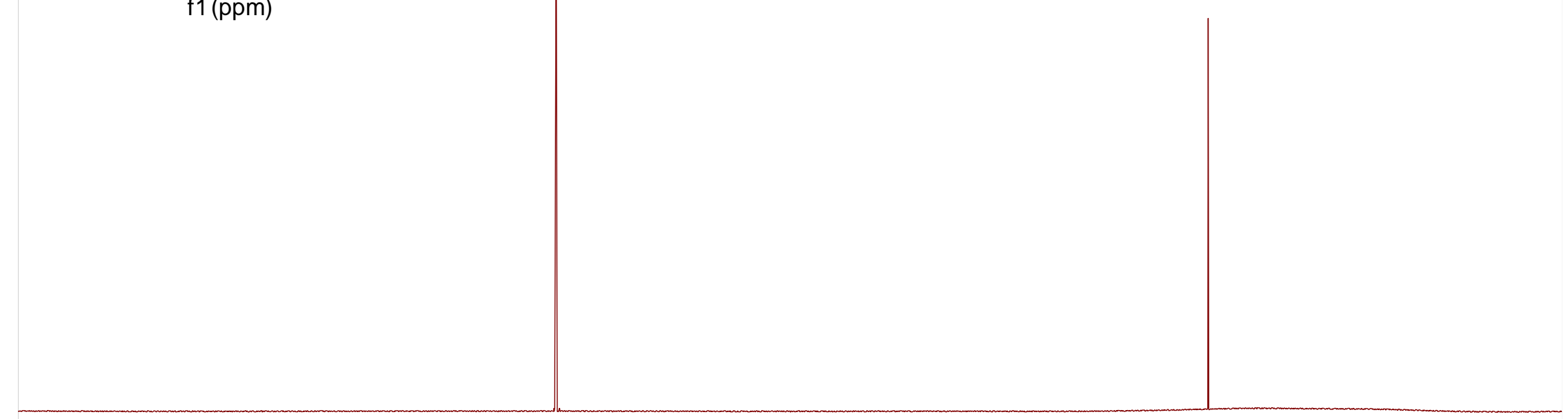

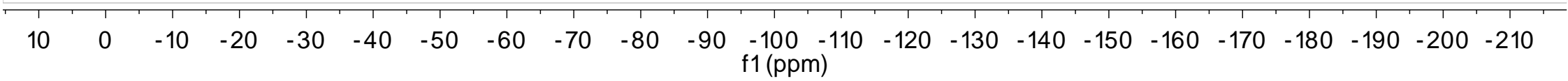




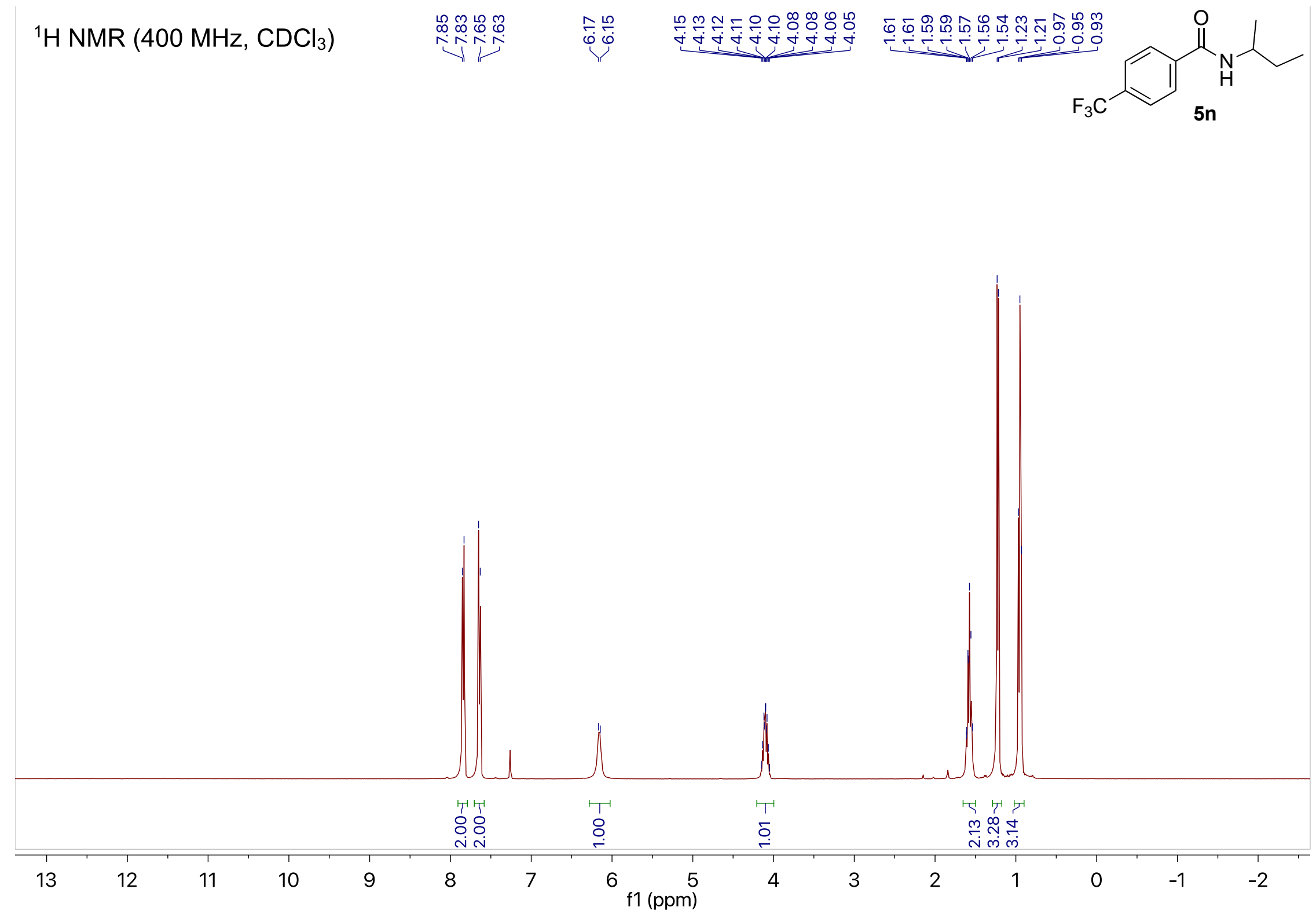


${ }^{13} \mathrm{C}\left\{{ }^{1} \mathrm{H}\right\}$ NMR $\left(101 \mathrm{MHz}, \mathrm{CDCl}_{3}\right)$

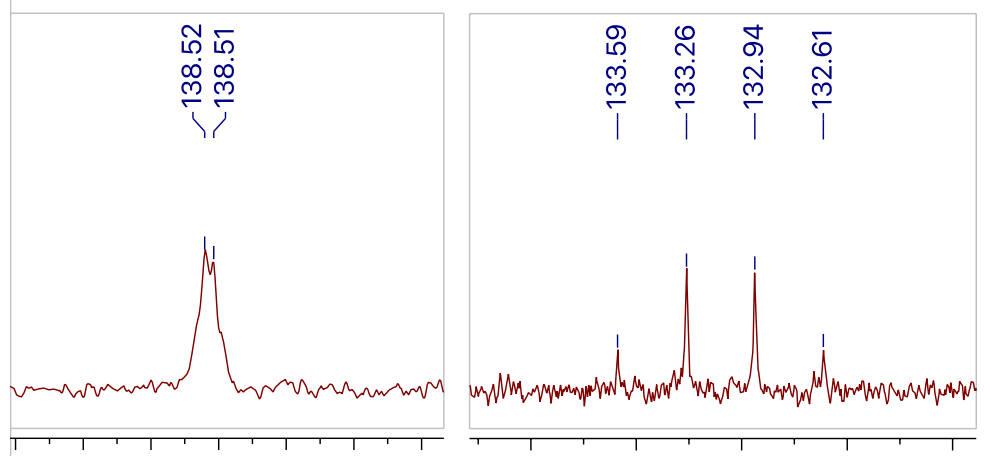

$\begin{array}{lllllllll}8.8 & 138.6 & 138.4 & 138.2 & 134.0 & 133.5 & 133.0 & 132.5 & 132.0\end{array}$ f1 (ppm)
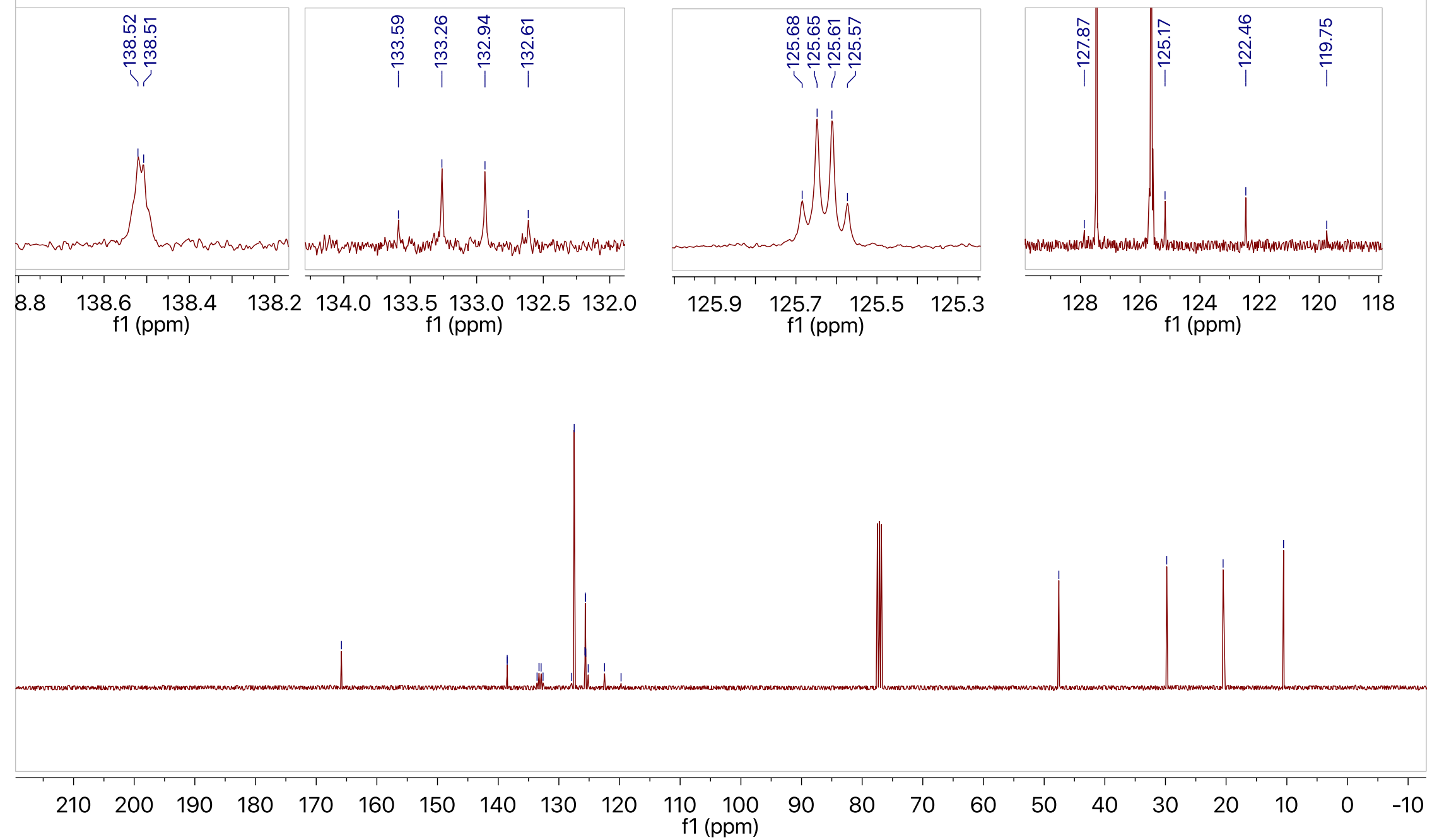


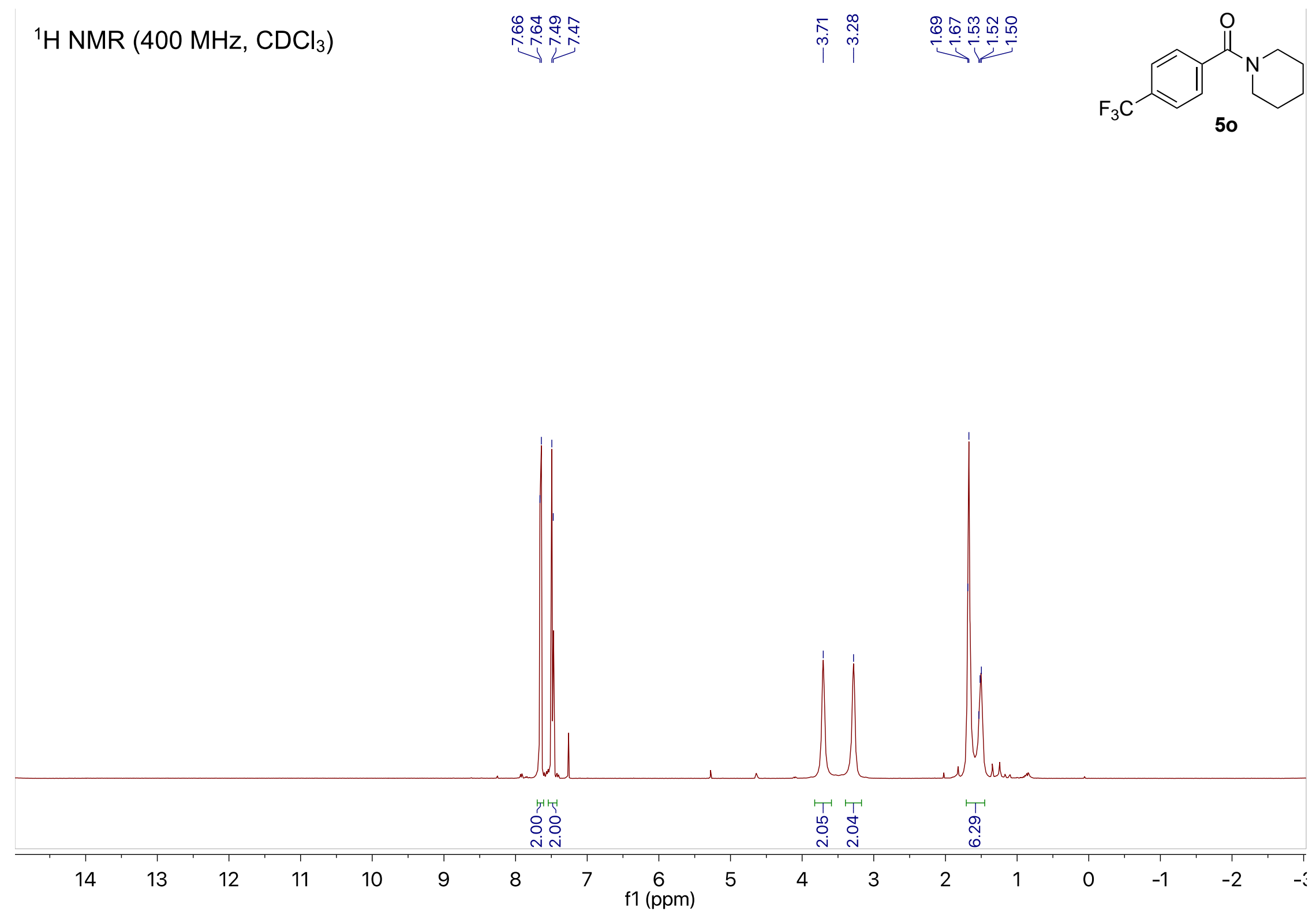




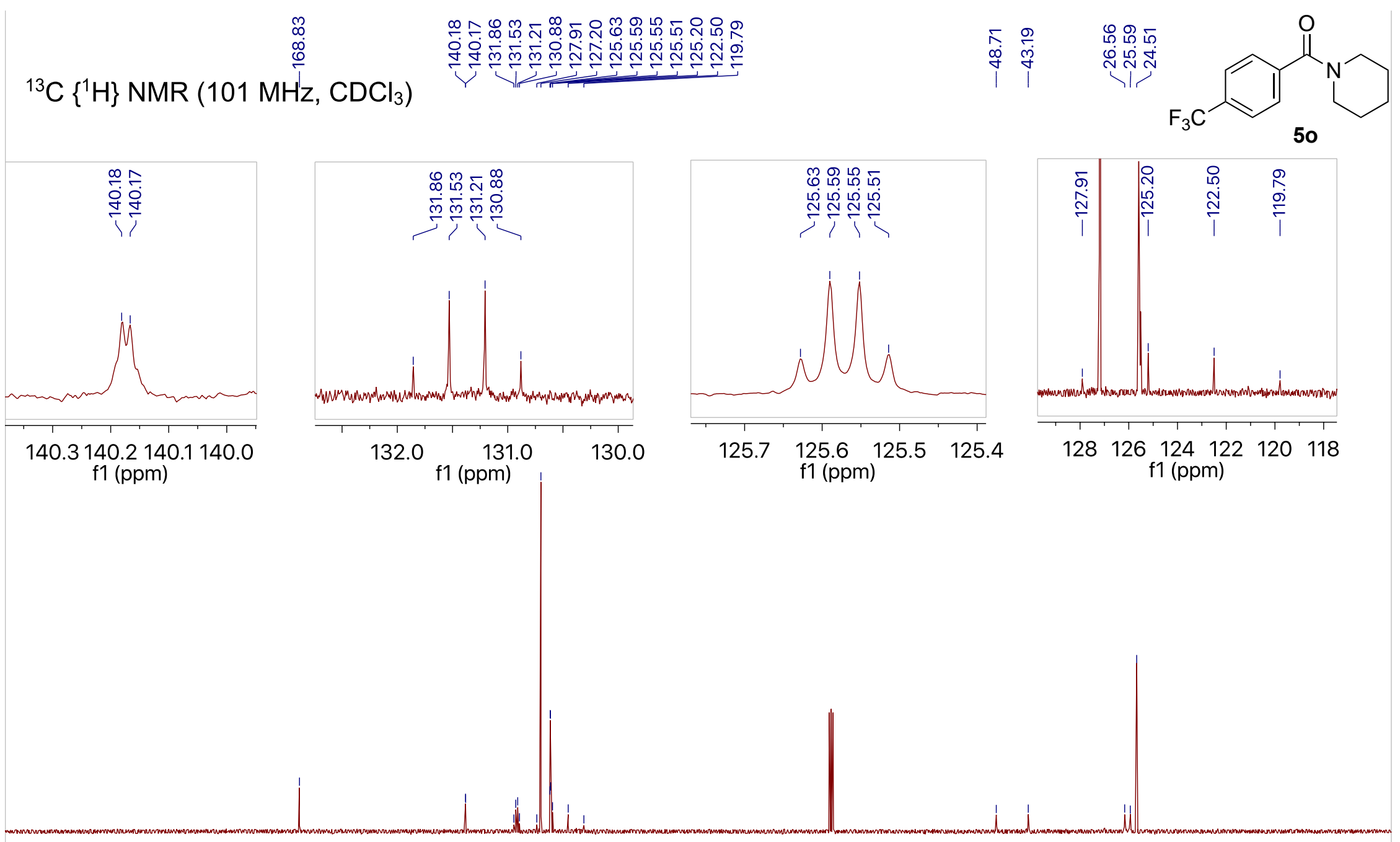

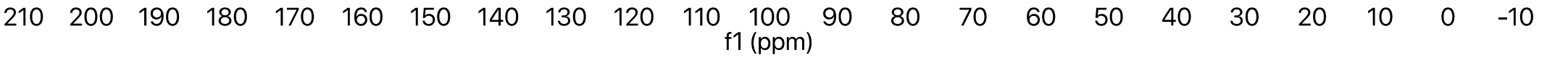


$\left.{ }^{19} \mathrm{~F} \mathrm{NMR} \mathrm{(376} \mathrm{MHz,} \mathrm{CDCl}_{3}\right)$
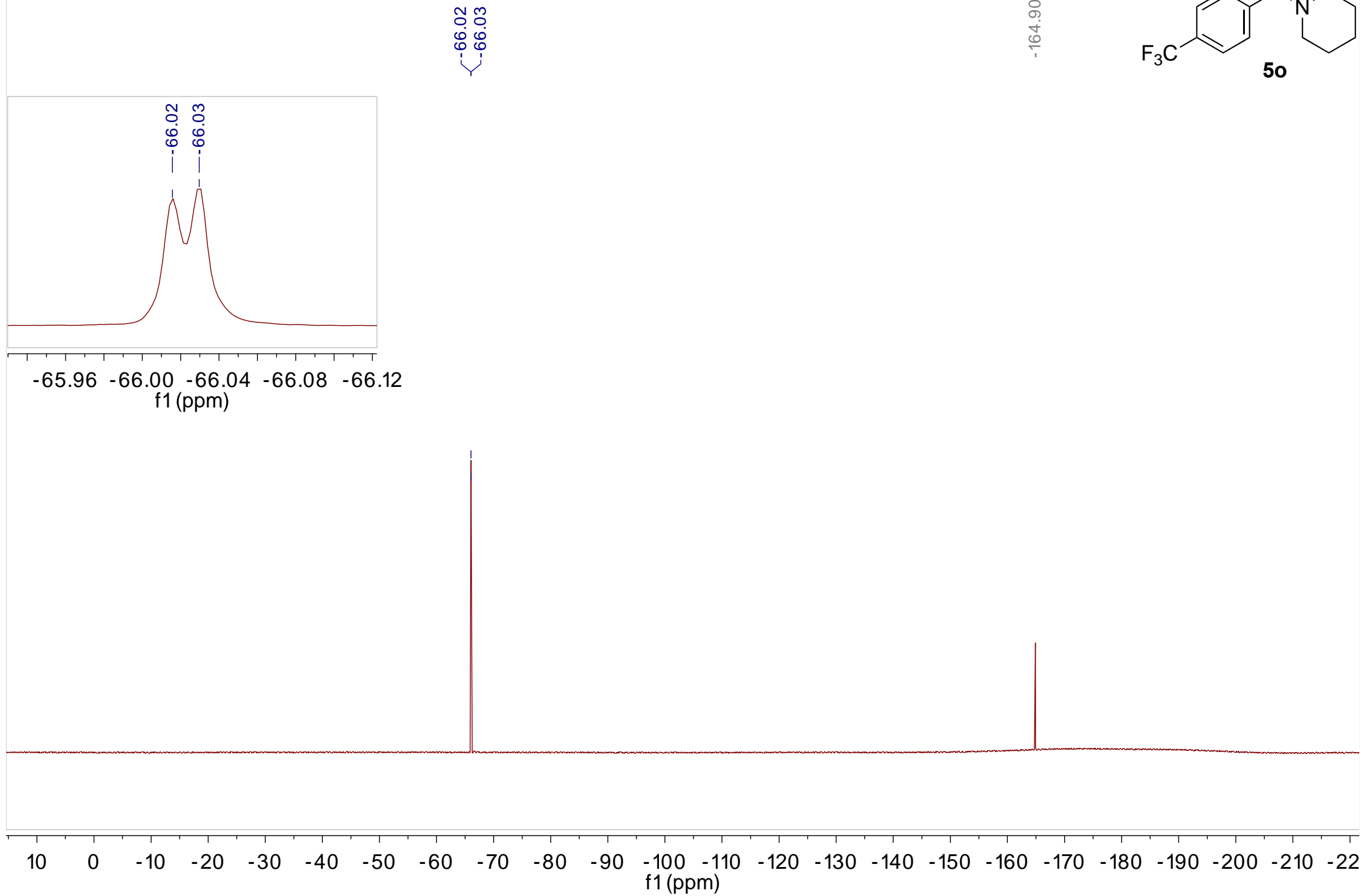

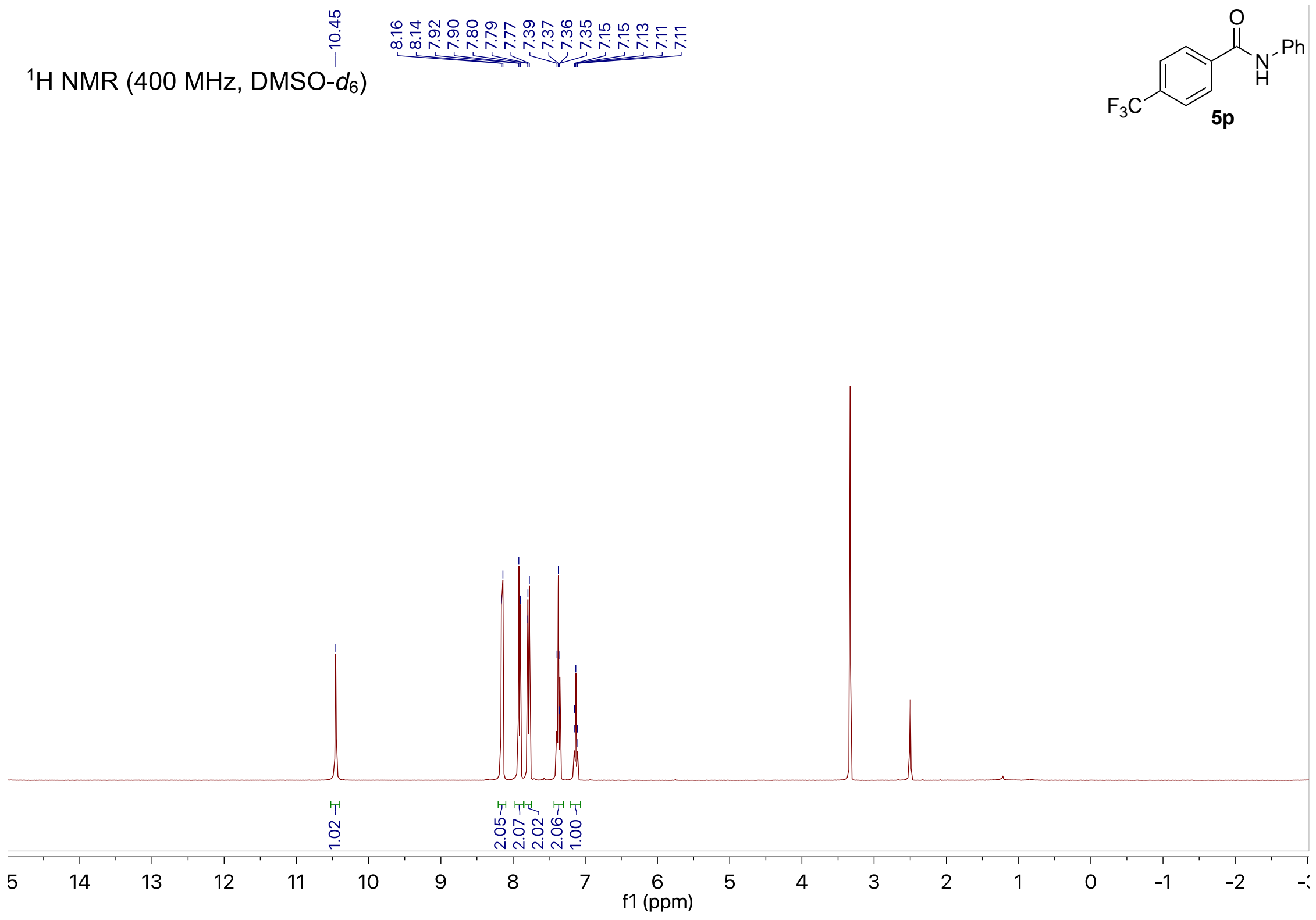


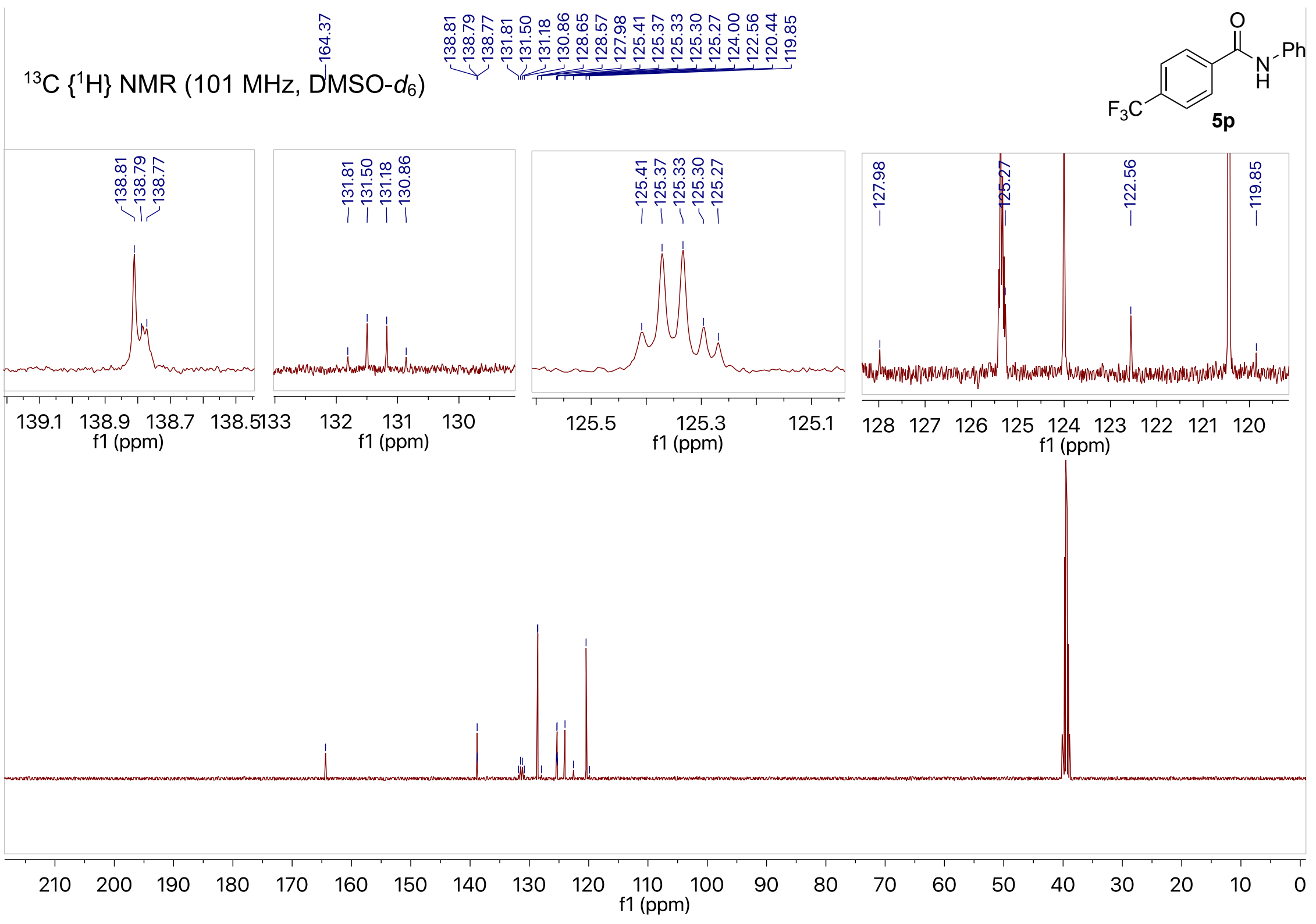


${ }^{19} \mathrm{~F}$ NMR $\left(376 \mathrm{MHz}\right.$, DMSO- $\left.d_{6}\right)$

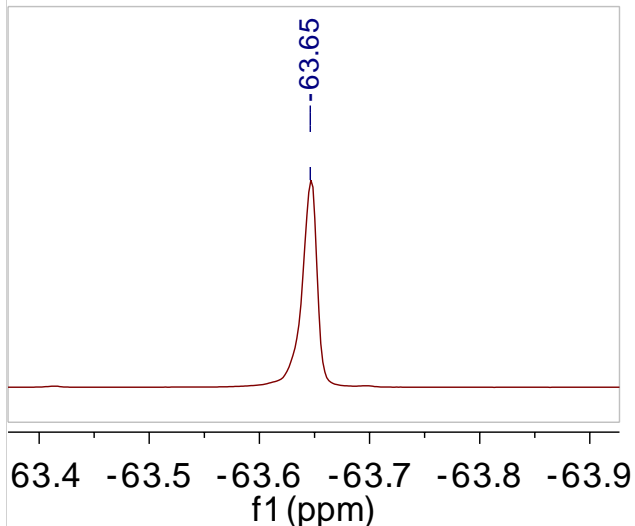
$\begin{array}{lllll}63.5 & -63.6 & -63.7 & -63.8 & -63.9\end{array}$

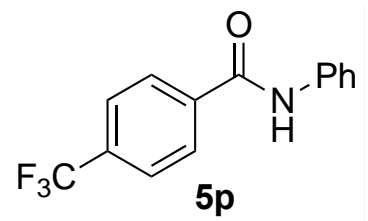

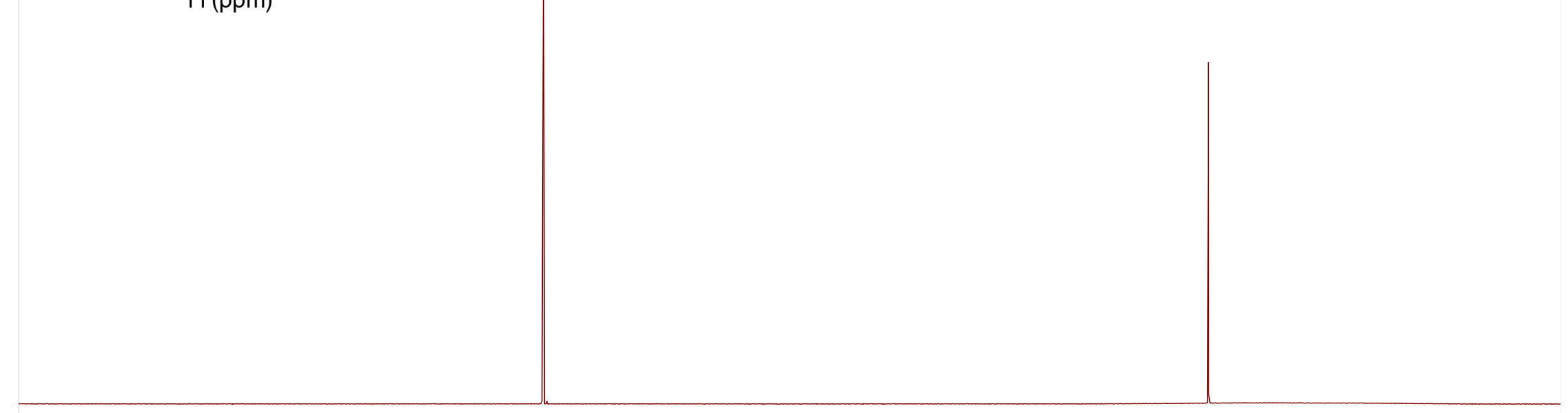

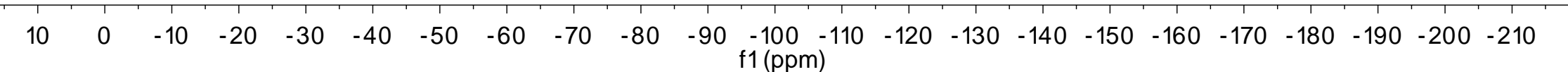




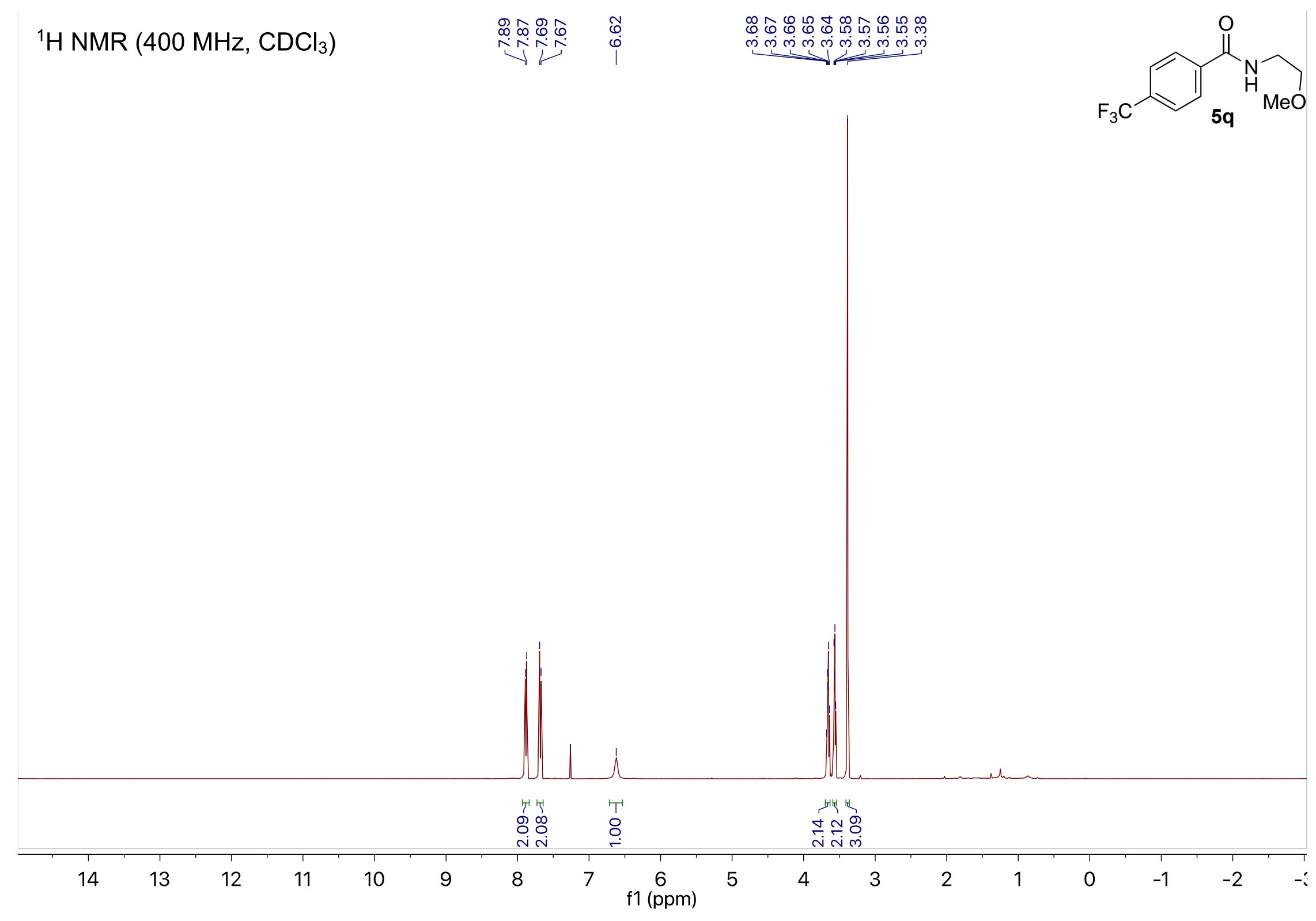



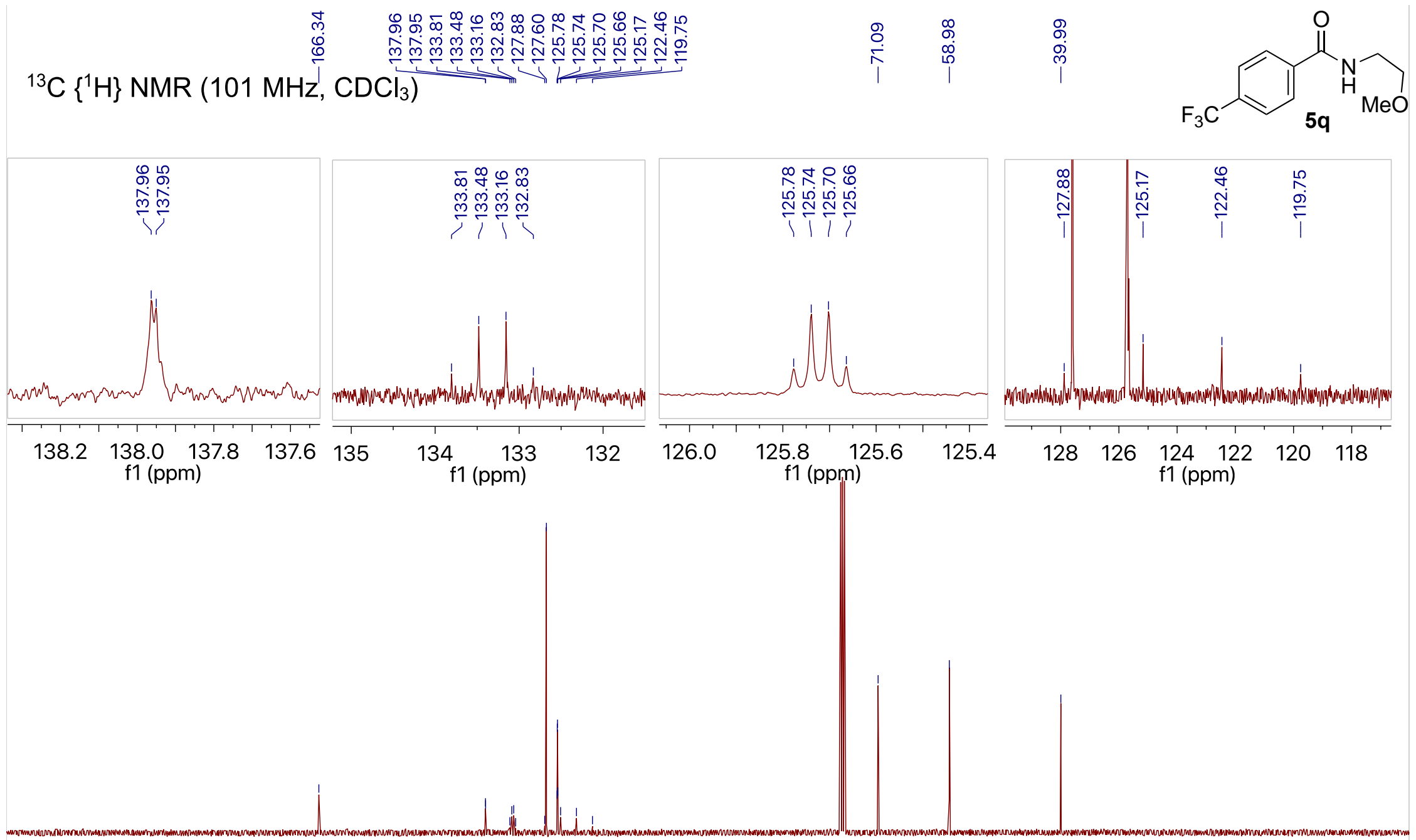

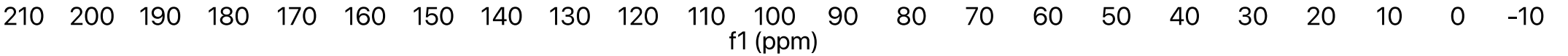


${ }^{19} \mathrm{~F} \mathrm{NMR}\left(376 \mathrm{MHz}, \mathrm{CDCl}_{3}\right)$

ํ.
$\dot{6}$
$\dot{1}$

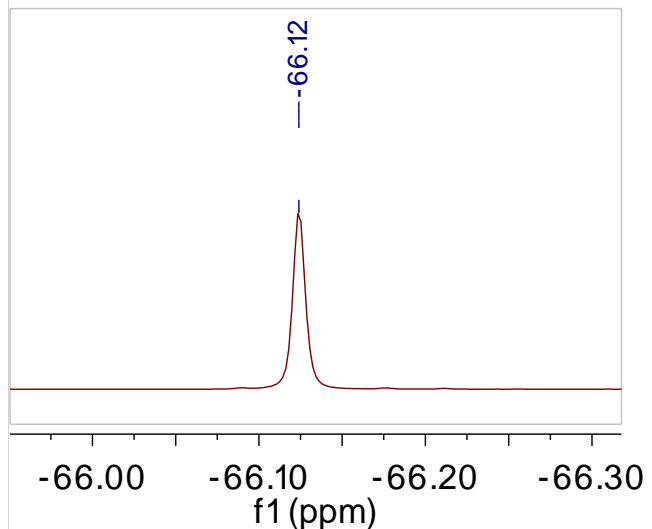

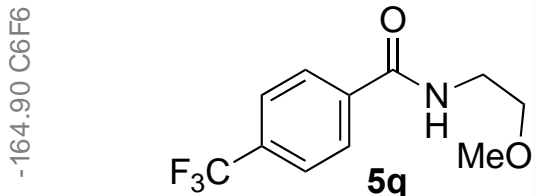

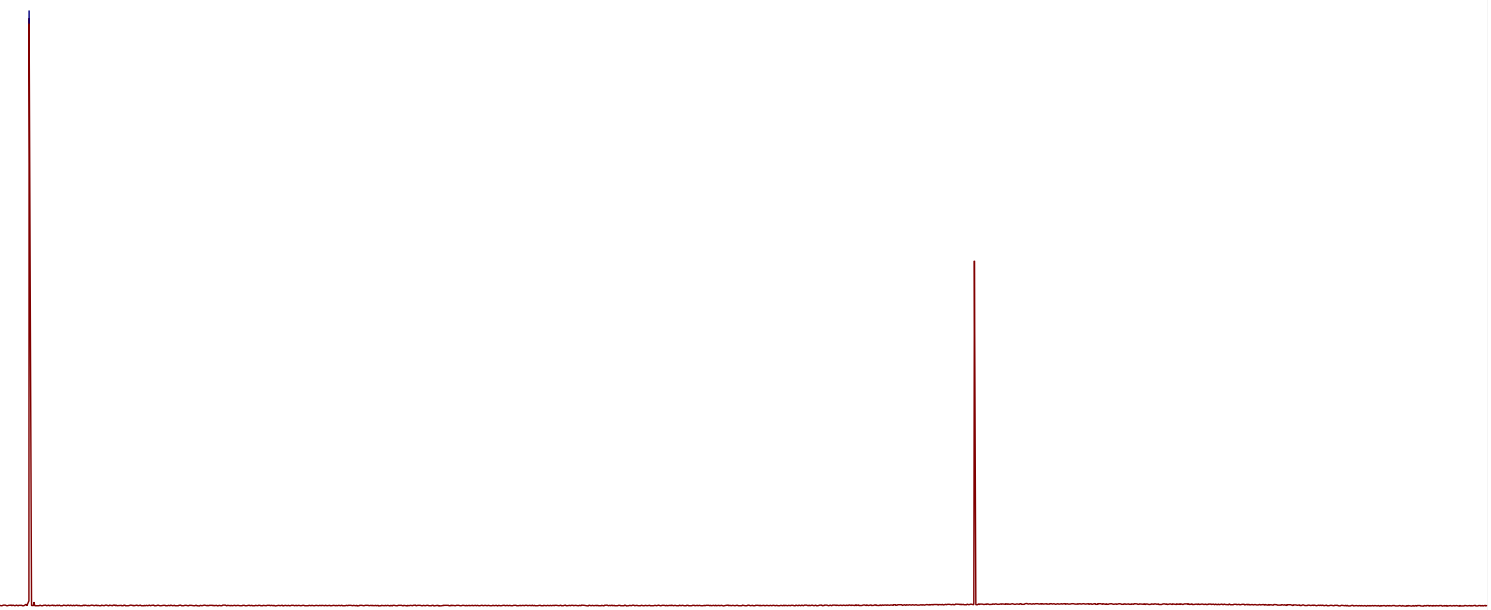

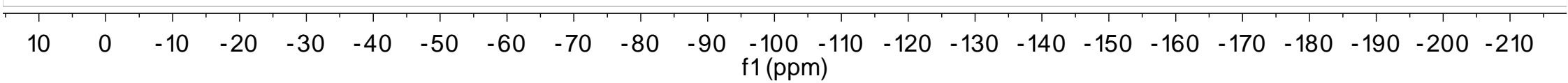




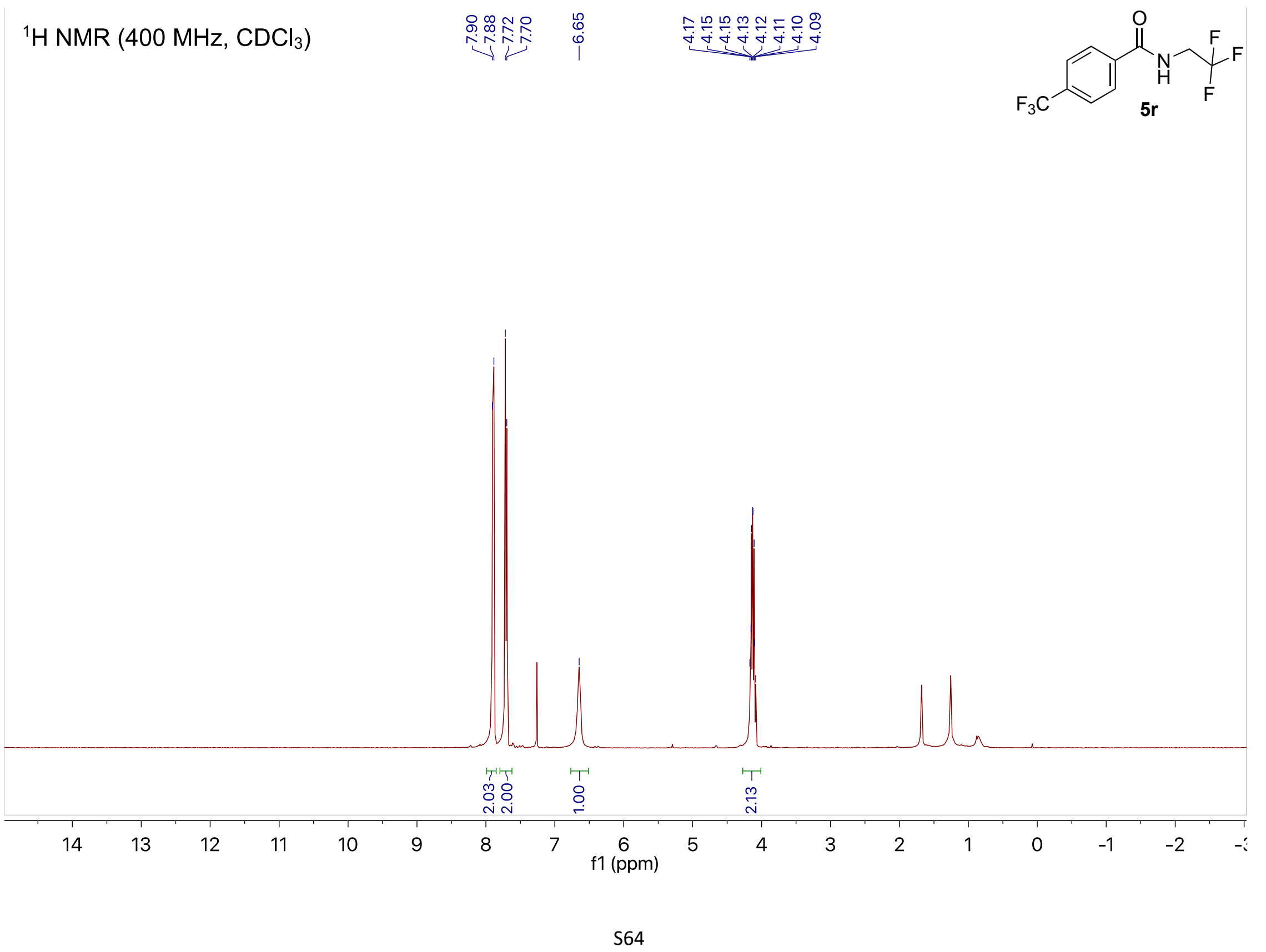



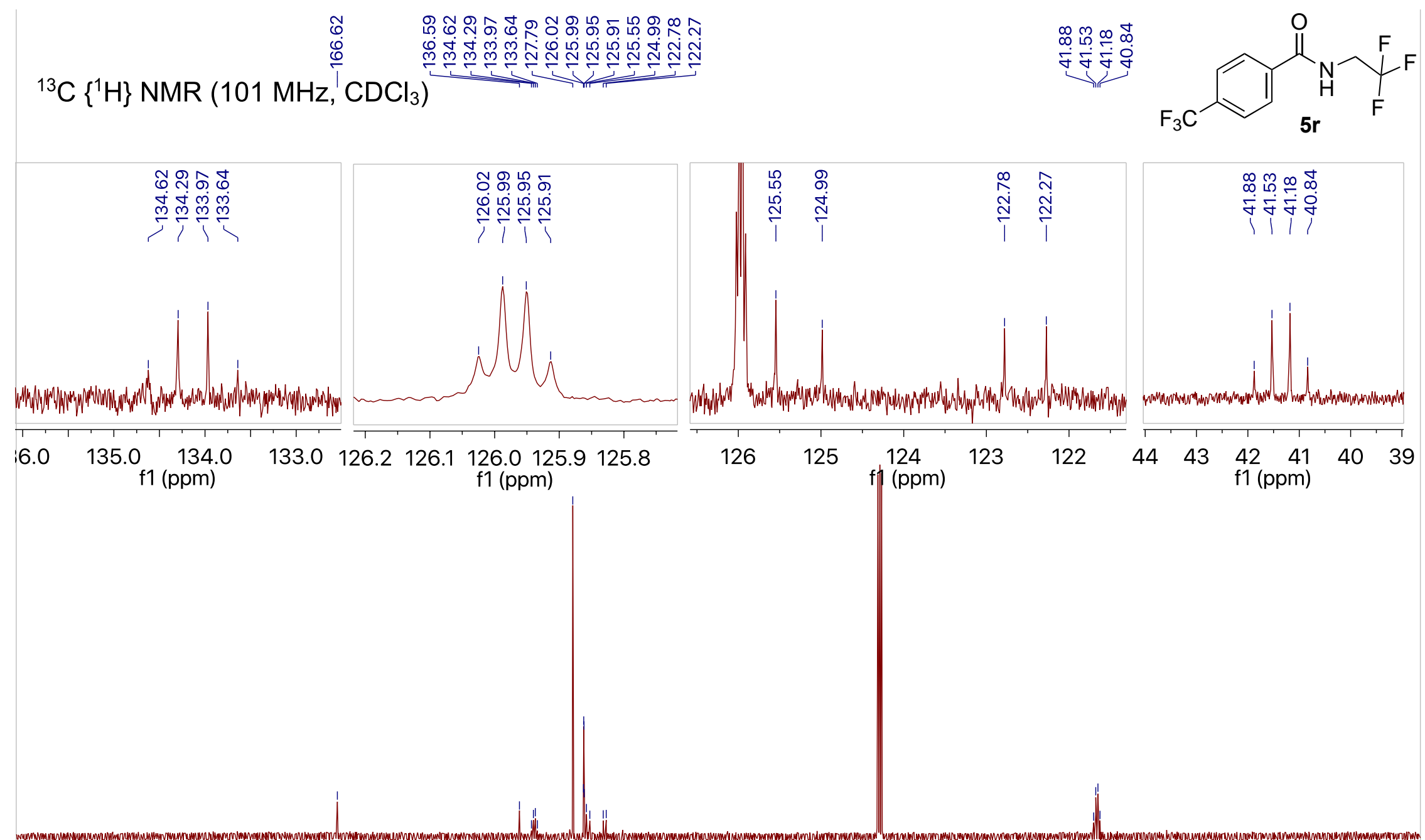

$\begin{array}{lllllllllllllllllllllll}210 & 200 & 190 & 180 & 170 & 160 & 150 & 140 & 130 & 120 & \begin{array}{r}110 \\ \mathrm{f} 1(\mathrm{ppm})\end{array} & 90 & 80 & 70 & 60 & 50 & 40 & 30 & 20 & 10 & 0 & -10\end{array}$ 
${ }^{19} \mathrm{~F} \mathrm{NMR}\left(376 \mathrm{MHz}, \mathrm{CDCl}_{3}\right)$

ஸ़

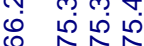

i i
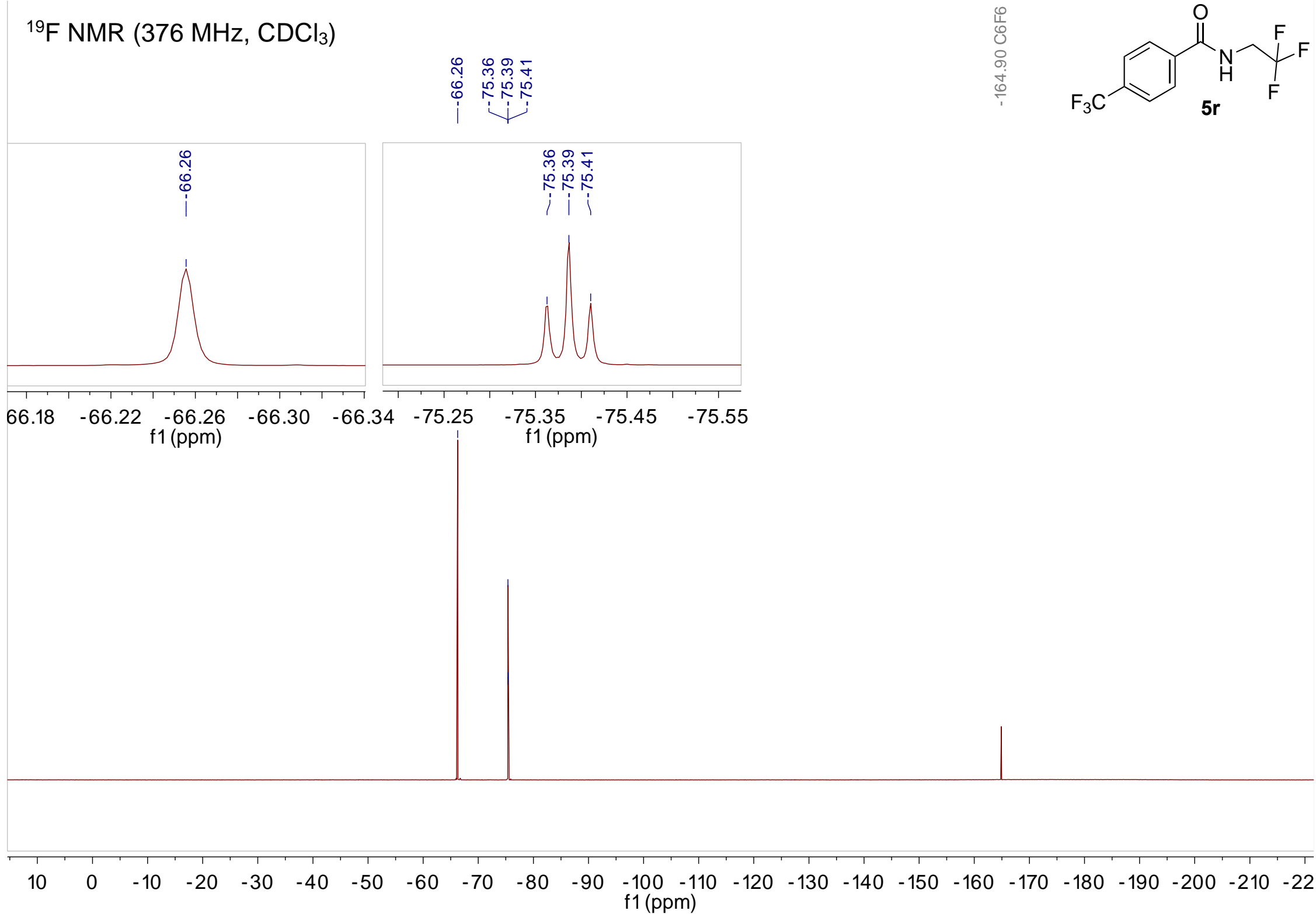


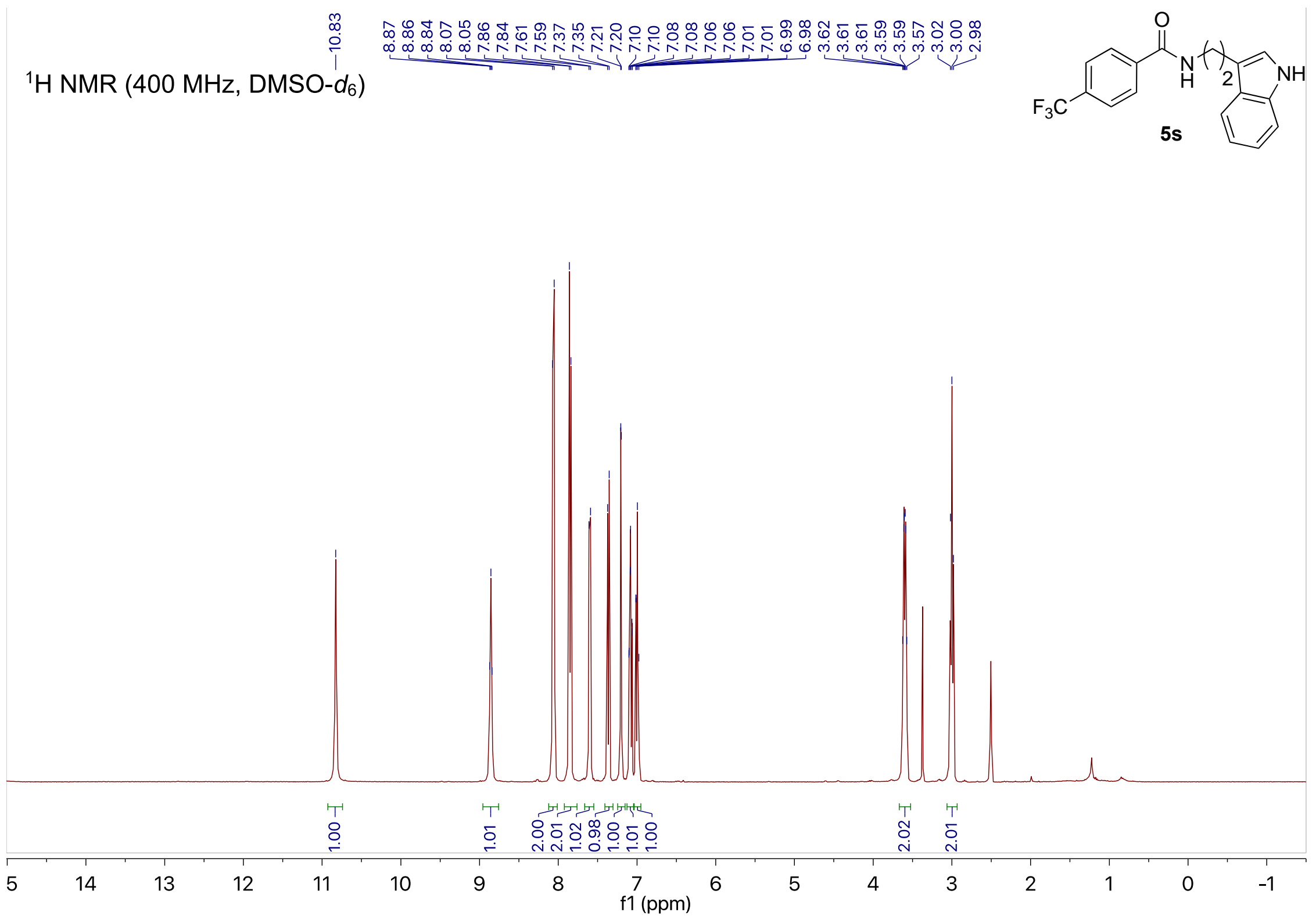




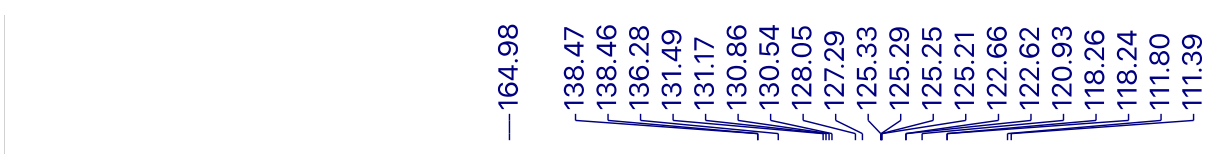

${ }^{13} \mathrm{C}\left\{{ }^{1} \mathrm{H}\right\} \operatorname{NMR}\left(101 \mathrm{MHz}, \mathrm{DMSO}-d_{6}\right)$
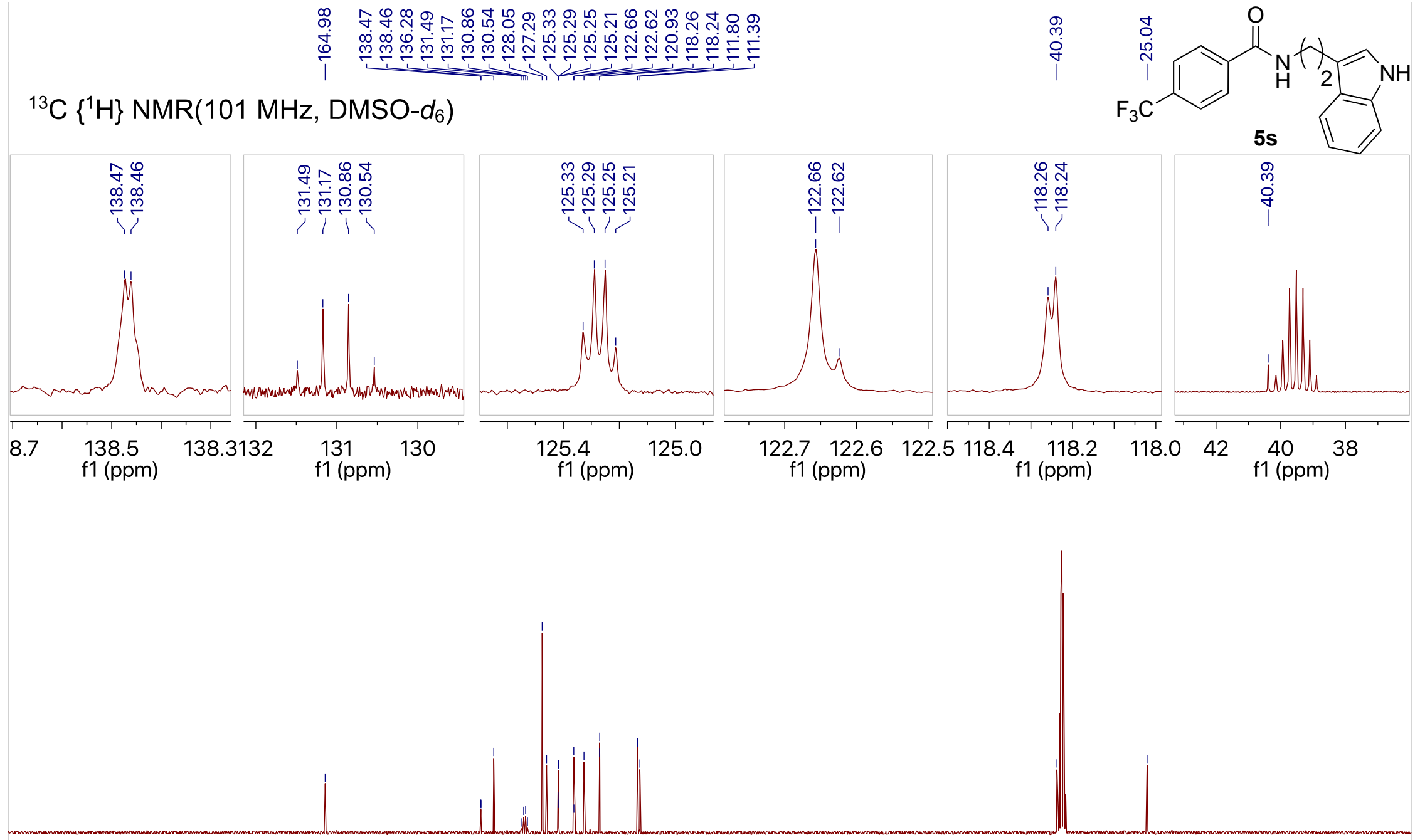

$\begin{array}{lllllllllllllllllllllll}210 & 200 & 190 & 180 & 170 & 160 & 150 & 140 & 130 & 120 & 110 & \begin{array}{c}100 \\ \mathrm{f} 1(\mathrm{ppm})\end{array} & 90 & 80 & 70 & 60 & 50 & 40 & 30 & 20 & 10 & 0 & -10\end{array}$ 
${ }^{19} \mathrm{~F}$ NMR $\left(376 \mathrm{MHz}, \mathrm{DMSO}-d_{6}\right)$

$\stackrel{1}{0}$
0
$\stackrel{0}{ }$
$\stackrel{1}{1}$

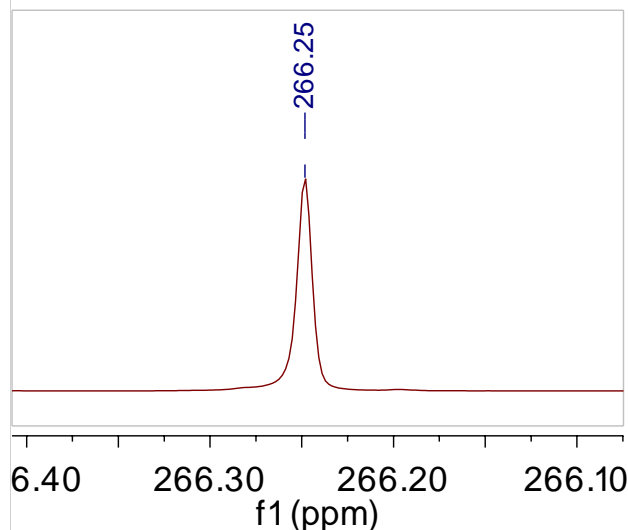

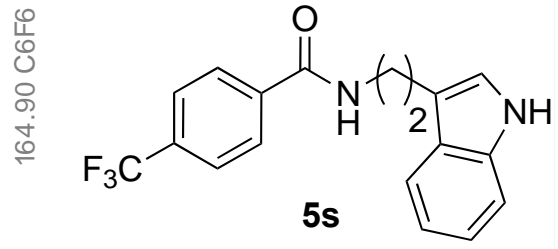

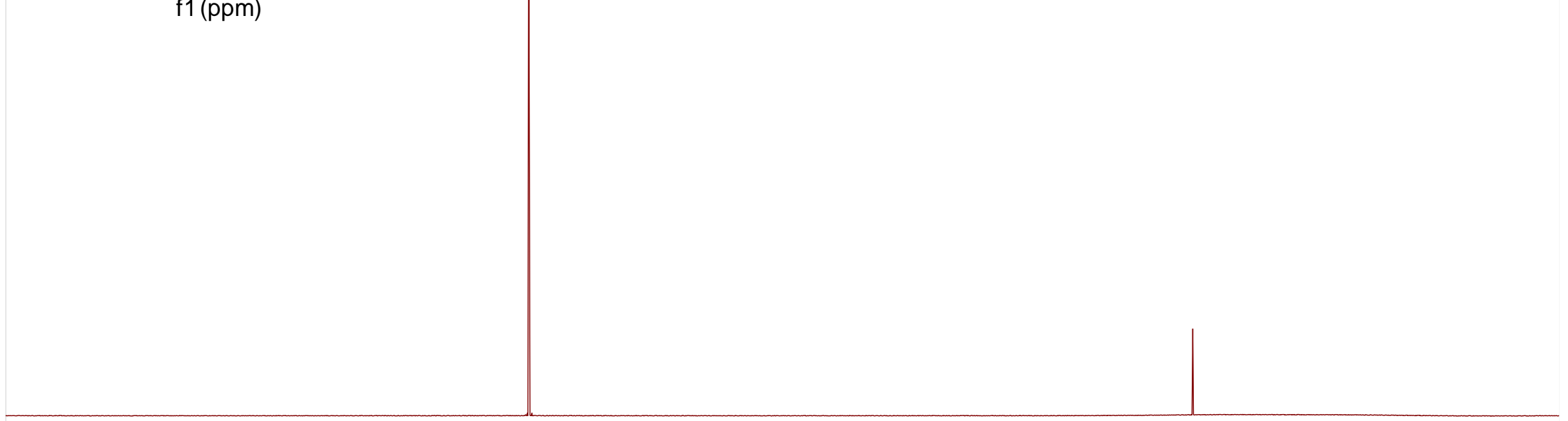

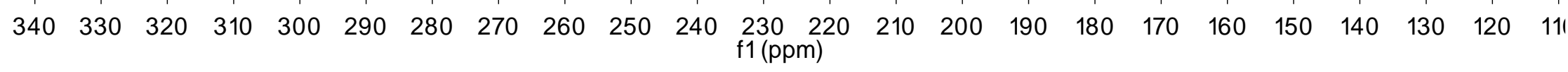




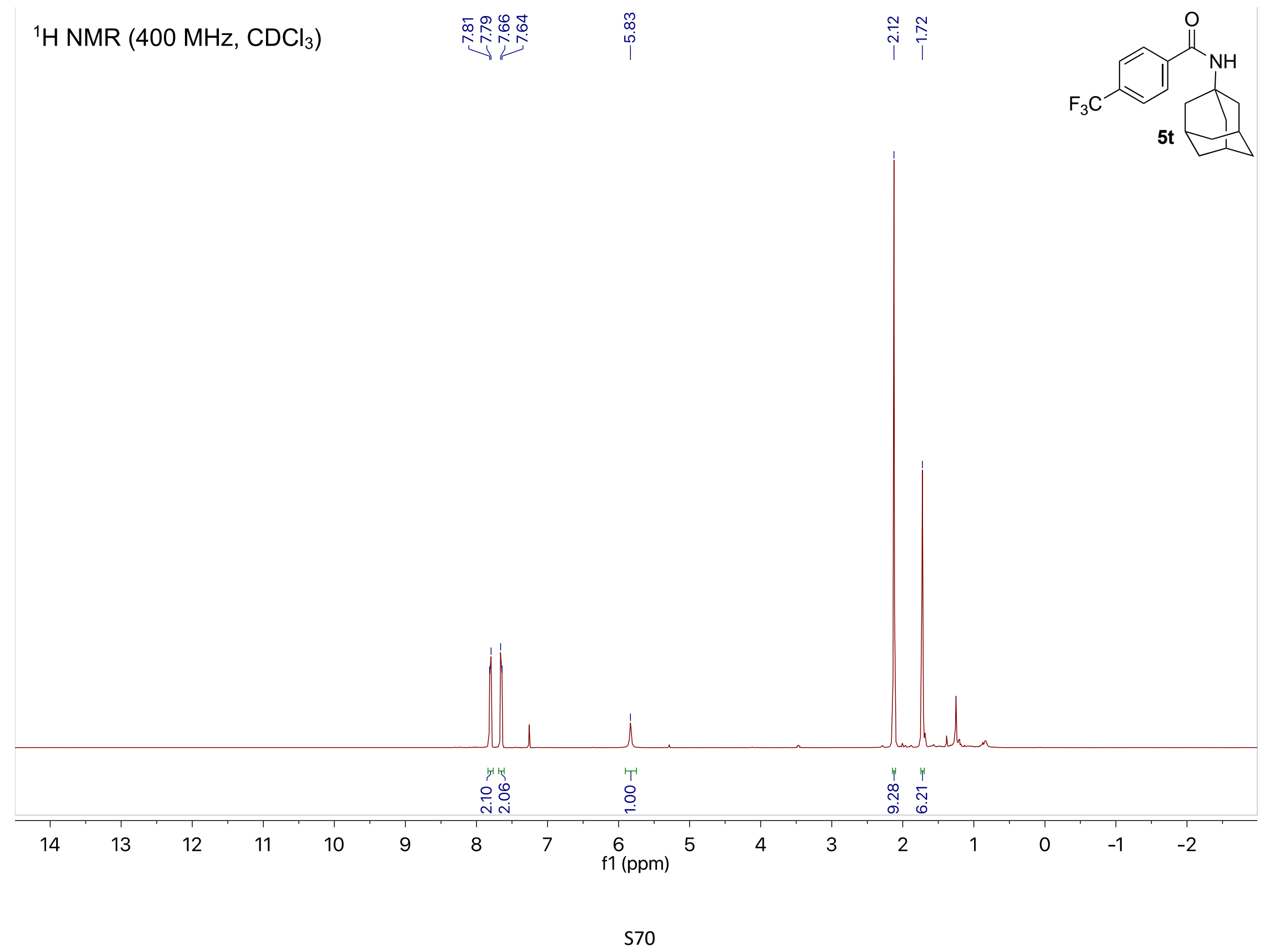




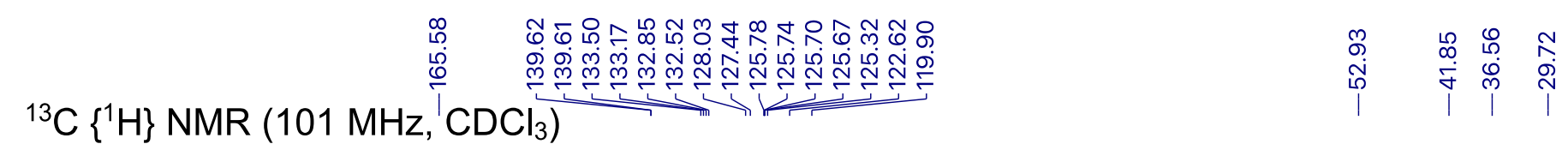
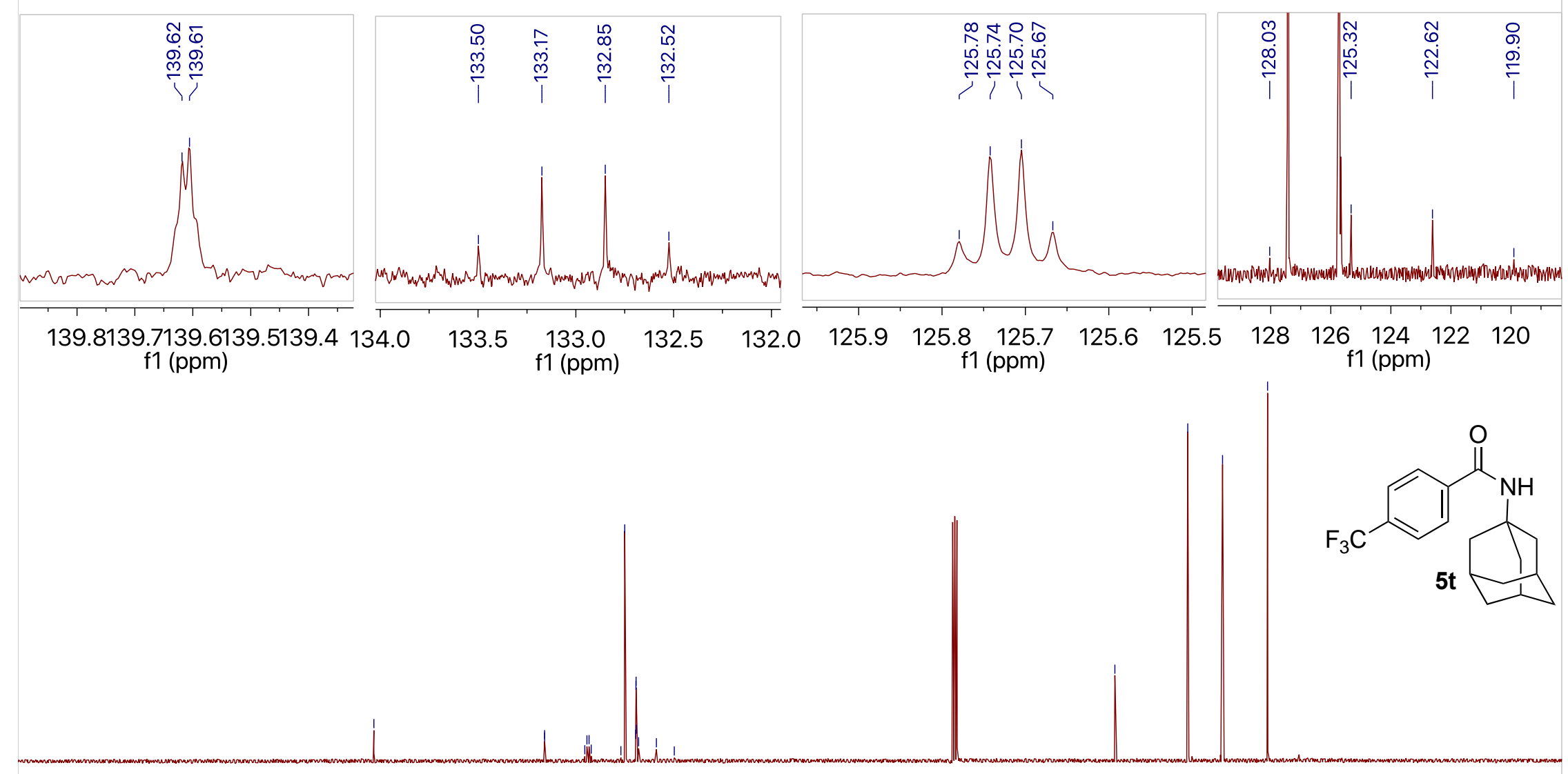

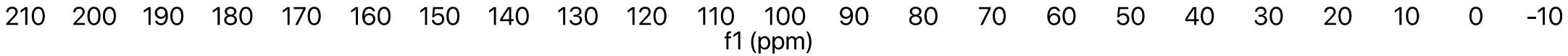


${ }^{19} \mathrm{~F} \mathrm{NMR}\left(376 \mathrm{MHz}, \mathrm{CDCl}_{3}\right)$

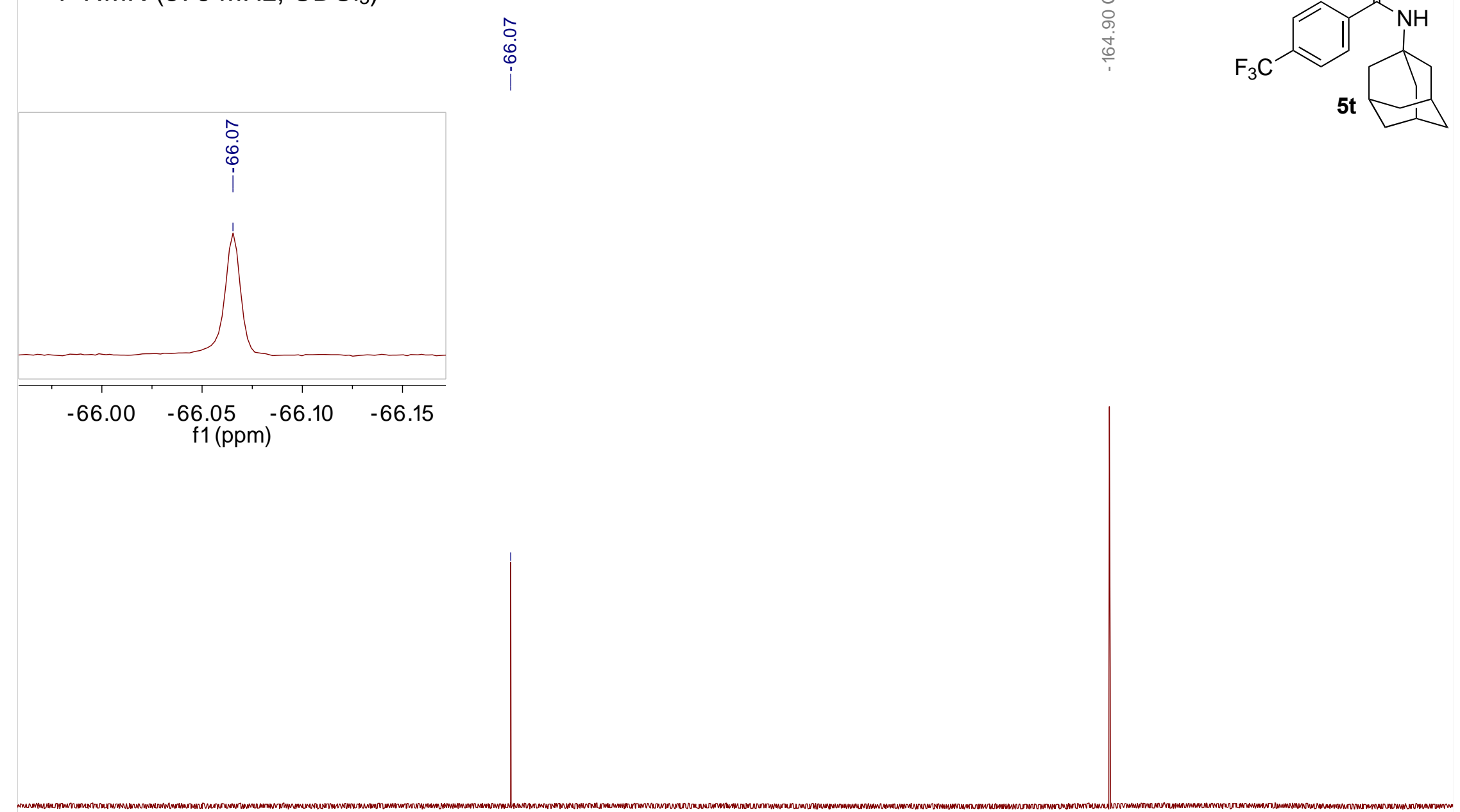

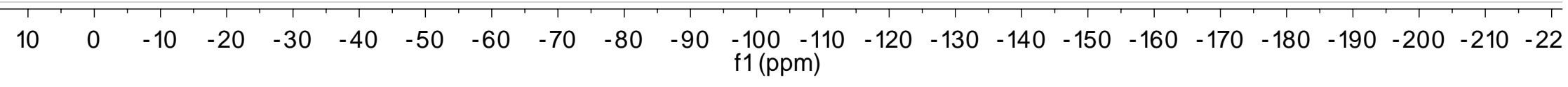




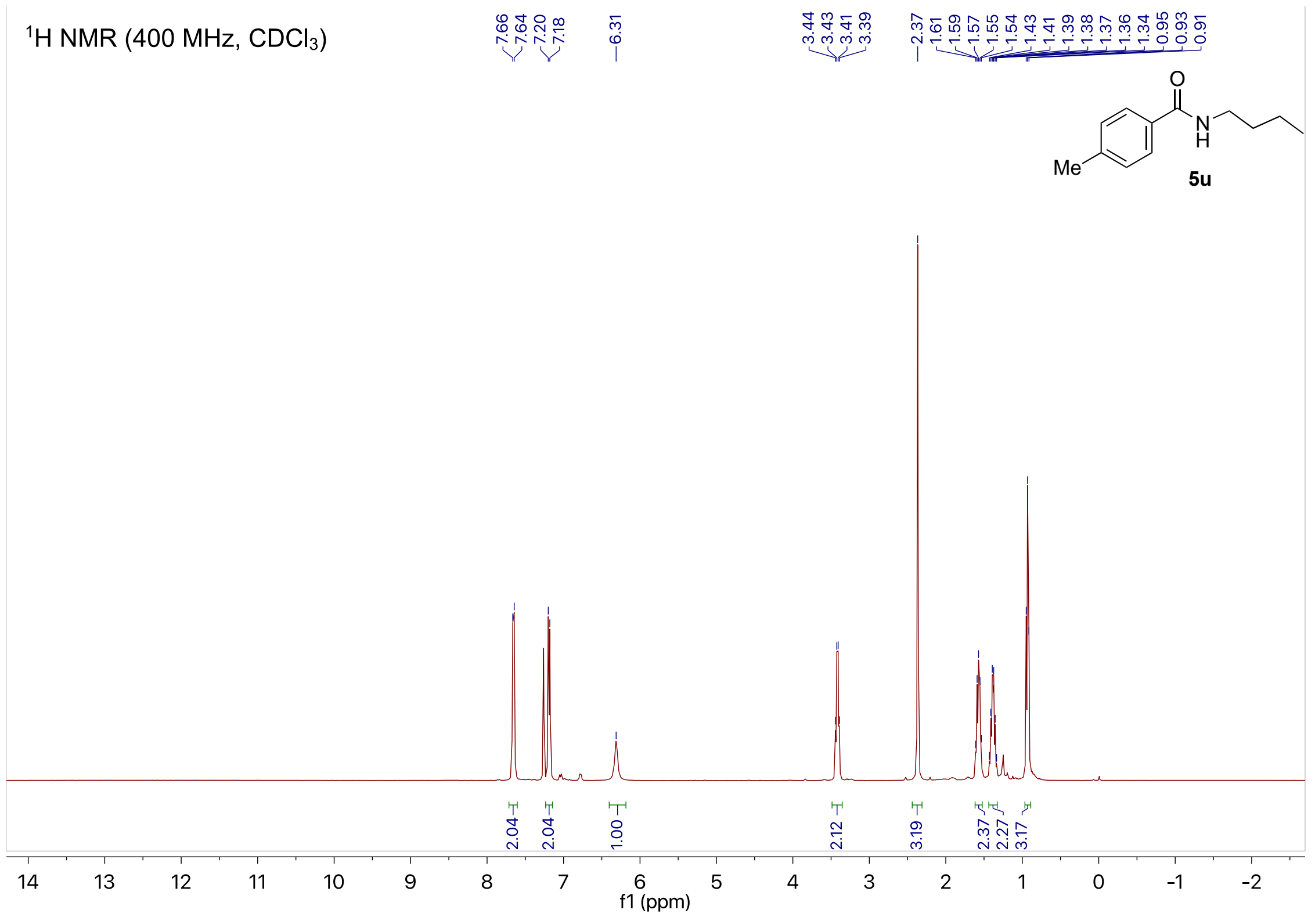


${ }^{13} \mathrm{C}\left\{{ }^{1} \mathrm{H}\right\}$ NMR (101 MHz, $\left.\mathrm{CDCl}_{3}\right)$

象
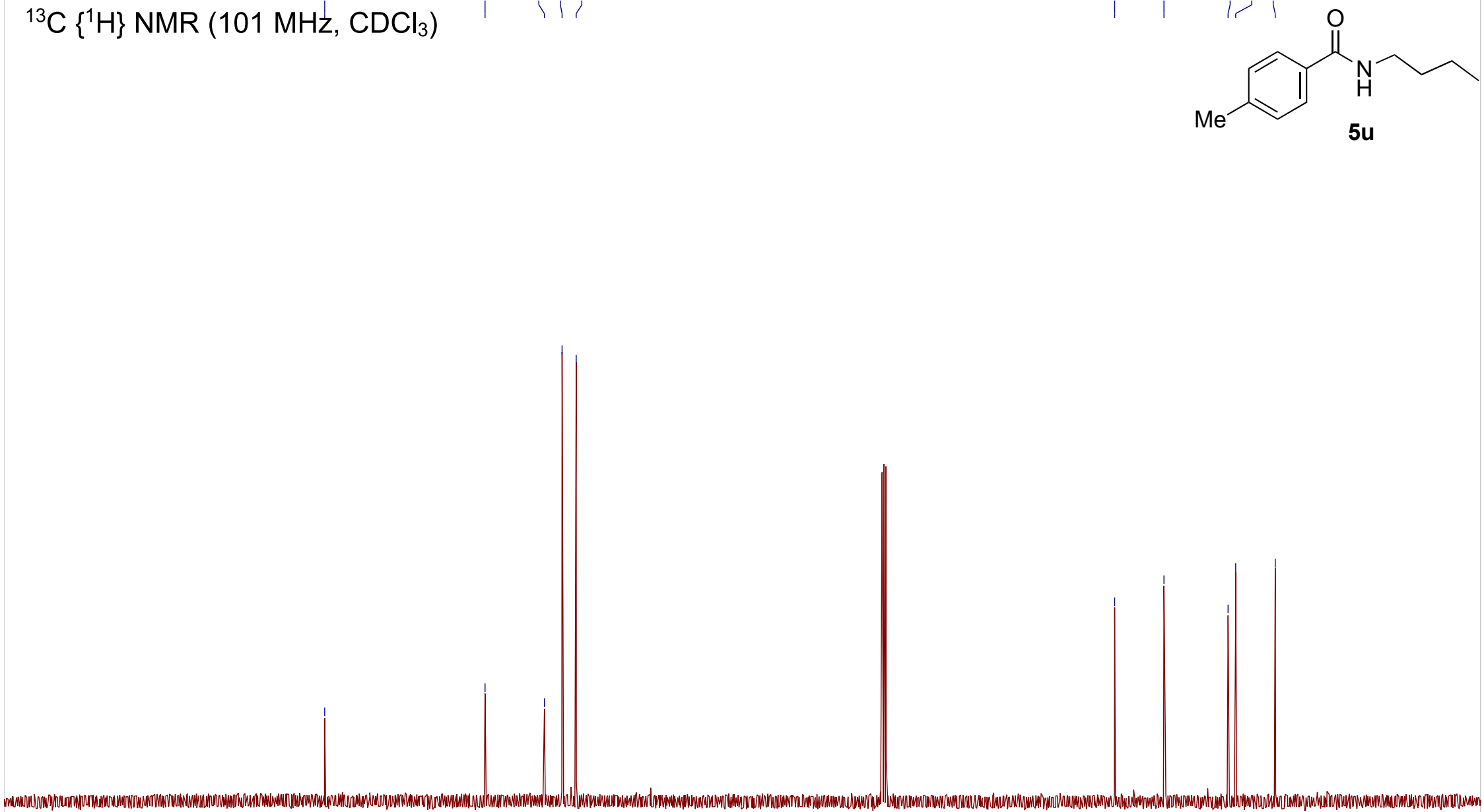

$210 \quad 200 \quad 190$

$180 \quad 170 \quad 160$

$\begin{array}{lll}50 & 140 \quad 130\end{array}$

$120110 \quad 100$

f1 (ppm) 


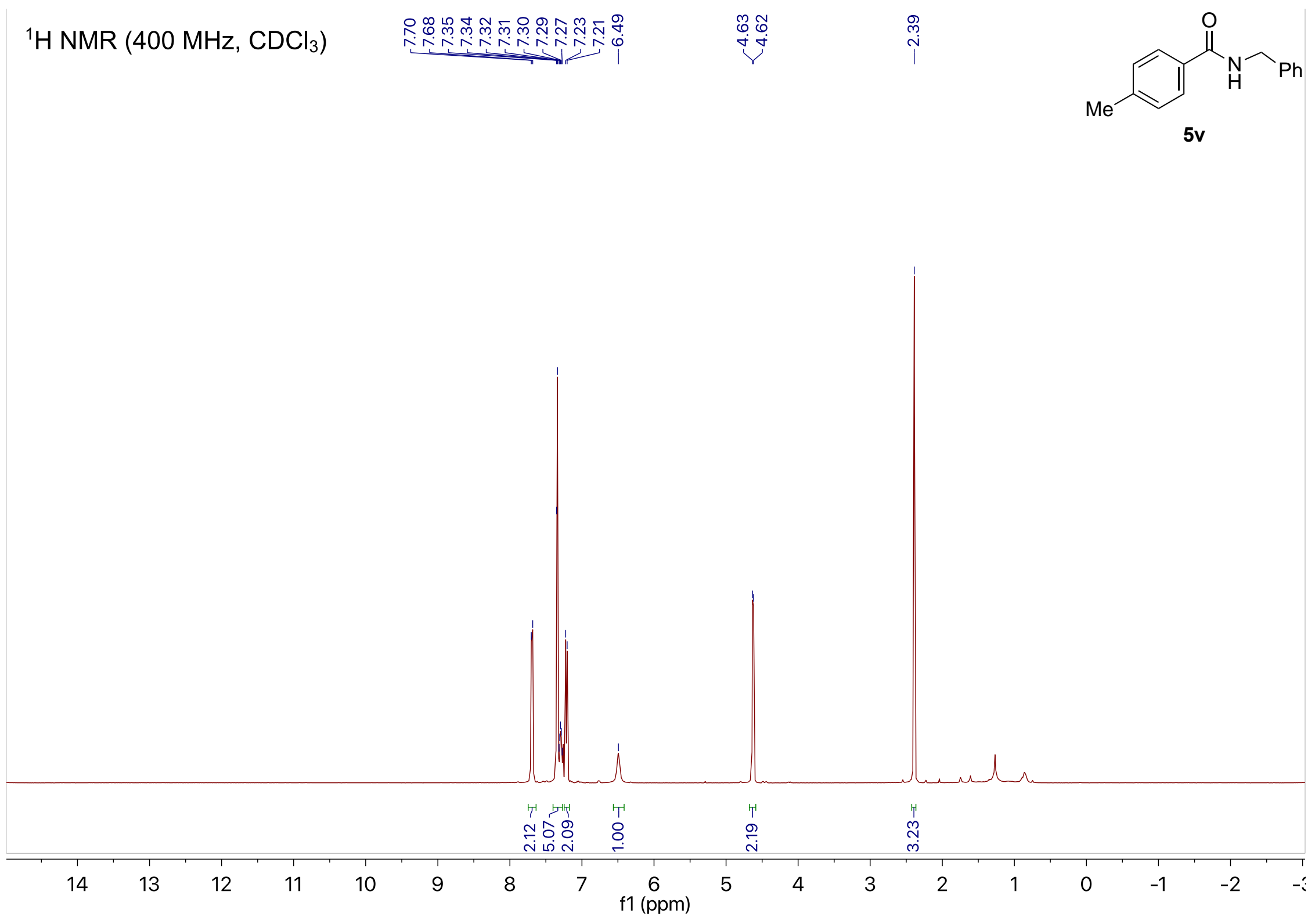




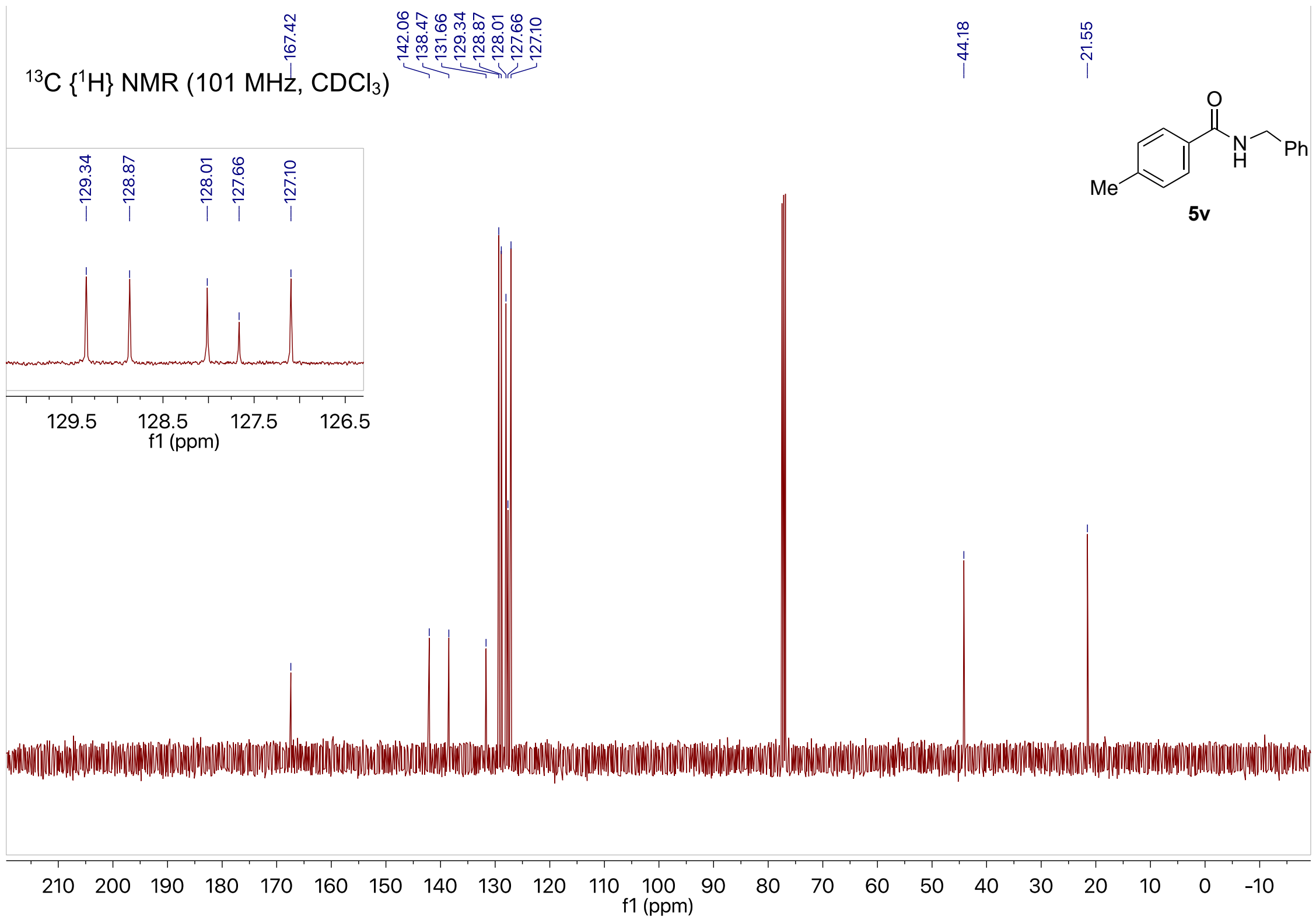

